\title{
NBSIR 73-146
}

\section{Outdoor Performance of Plastics $X$. Final Update of Weathering Data}

Walter J. Rossiter, Jr.

Center for Building Technology Institute for Applied Technology

National Bureau of Standards

March 1973

Final Report

\section{Prepared for}

Manufacturing Chemists Association 1825 Connecticut Avenue, N. W.

Washington, D. C. 20009 


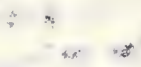


NBSIR 73-146

\section{OURDOOR PERFORMANCE OF PLASTICS \\ $X$. FINAL UPDATE OF WEATHERING DATA}

Walter J. Rossiter, Jr.

Center for Building Technology Institute for Applied Technology

National Bureau of Standards

March 1973

Final Report

Prepared for

Manufacturing Chemists Association

1825 Connecticut Avenue, N. W.

Washington, D. C. 20009

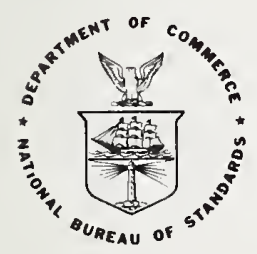

U. S. DEPARTMENT OF COMMERCE, Frederick B. Dent, Secretary

NATIONAL BUREAU OF STANDARDS, Richard W. Roberts, Director 


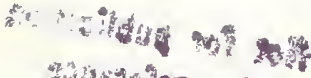

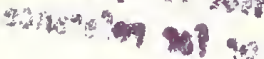




\section{Abstract}

Twenty plastics samples have been weathered in Arizona, Florida, and Washington, D. C. for 72 months. The weathering of these samples has been followed by measuring changes in the specimen's color, tensile, flexure, gloss, and haze properties. Computer-generated graphs of these changes with time are presented. 

This is the final updating of the Manufacturing Chemists' Association's (MCA) project which studied the outdoor performance of plastics. Since 1966, twenty plastics samples (Table I) have been exposed outdoors at three different exposure sites (Table II) which represent varying climatic conditions encountered in the United States. The MCA project and preliminary results have been described in a series of National Bureau of Standards Reports. These reports [1-9]*, all entitled Outdoor Performance of Plastics, have the following subtitles:

I. Introduction and Color-Change [1].

II. Tensile and Flexural properties [2].

III. Statistical Model for Predicting Weatherability [3].

IV. Significance of Climate [4].

V. Surface Roughness [5].

VI. Electrical Properties [6].

VII. Haze and Gloss [7].

VIII. First Update of Weathering Data [8].

IX. Second Update of Weathering Data [9].

Changes in those properties 1 isted in the above subtitles were the means for determining the outdoor performance of the twenty samples. A computer was used for data storage and retrieval. Graphs of property vs. outdoor exposure time were generated by the computer. These graphs were reproduced in the various reports as the method of reporting property changes.

* Numbers in brackets refer to references which are found in the back of this report. 
From the preliminary results, it was decided that color, tension, flexure, gloss, and haze would be the significant properties to follow for the remainder of the project. The samples remaining have now been exposed outdoors for 72 months. These five properties have been measured for each plastic. The physical testing was again performed by MCA member companies (Table III), and the test results sent here to the National Bureau of Standards for incorporation with the earlier data. Although these data were originally reported in three separate NBS reports $[1,2,7]$, the data through 72 months for the five properties are a11 presented in this single report.

Experimental details are not given here; such details can be found in the earlier reports. Again, the data are presented as reproductions of the computer-generated graphs (Figures 1-81B). These graphs differ from the earlier ones in that the X-axis (time in months) has been expanded to 72 months. Thus, the data points are compressed when compared to those of the original graphs.

This report includes all previous data. Some plastics have failed in the field and obviously there are no additional data for these samples. Graphs with the expanded time have been reproduced for those samples for which there are no additional data. This report is meant to be a replacement for the two previous supplemental ones $[8,9]$. The weathering of the plastics can be followed without reference to them. The graphs are identical except for the additional data.

The color data presented in the first report [1] were erroneous. These data were corrected in the first supplemental report [8]. No 
comparisons can be made between the graphs for color change in the first report and any of the updates.

This report is only intended as a presentation of the new data. No discussion of the accumulated data is presented. 


\section{$\underline{\text { References }}$}

[1] "Outdoor Performance of Plastics. I. Introduction and Color-Change", J. E. Clark, N. E. Green, and P. Giesecke, NBS Report 非9912, Sept. 1968 .

[2] "Outdoor Performance of Plastics. II. Tensile and Flexural Properites", J. E. Clark, G. E. Gulmer, R. C. Neuman, and J. A. Slater, NBS Report 非10014, March 1969.

[3] "Outdoor Performance of Plastics. III. Statistical Model for Predicting Weatherability", J. E. Clark and J. A. Slater, NBS Report 非10016, Oct. 1969 .

[4] "Outdoor Performance of Plastics. IV. Significance of C1imate", J. E. Clark and C. Bal Krishna, NBS Report 非10156, Jan. 1970.

[5] "Outdoor Performance of Plastics. V. Surface Roughness", J. E. Clark, C. Bal Krishna, H. C. Gunst, and J. R. Dagon, NBS Report 非10179, March 1970 .

[6] "Outdoor Performance of Plastics. VI. Electrical Properites", J. E. Clark, J. A. Slater, and V. L. Bergeron, NBS Report 非10185, March 1970.

[7] "Outdoor Performance of Plastics. VII. Haze and Gloss", J. E. Clark, C. Bal Krishna, G. C. Claver, and F. H. McTigue, NBS Report 非10188, March 1970.

[8] "Outdoor Performance of Plastics. VIII. First Update of Weathering Data", W. J. Rossiter, Jr. and W. D. Hayes, Jr., NBS Report 非10479, September 1971 .

[9] "Outdoor Performance of Plastics. IX. Second Update of Weathering Data", W. J. Rossiter, Jr., NBS Report 非10856, May 1972 . 
Table I

List of Twenty Plastics

Base Polymer

Pol ye thylene $\underline{\text { Plastic }}$

Translucent - 1 mil

- $60 \mathrm{mil}$

Poly (methyl methacrylate)

Clear - $60 \mathrm{mil}$

Poly (vinyl fluoride)

Clear - 1 mil

Poly (ethylene terephthalate)

Clear - $5 \mathrm{mil}$

Polyester/x-1 inked

Clear - $60 \mathrm{mil}$

Poly (vinyl chloride)

\begin{tabular}{|c|c|}
\hline $\begin{array}{l}\text { Clear } \\
\mathrm{Ba}-\mathrm{Cd}\end{array}$ & $\begin{array}{l}-4 \mathrm{mil} \\
-10 \mathrm{mil} \\
-60 \mathrm{mil}\end{array}$ \\
\hline $\begin{array}{l}\text { Clear } \\
\mathrm{Sn}\end{array}$ & $\begin{array}{l}-4 \mathrm{mil} \\
-10 \mathrm{mil} \\
-60 \mathrm{mil}\end{array}$ \\
\hline Clear & $-60 \mathrm{mil}$ \\
\hline $\begin{array}{l}\text { White } \\
\mathrm{Ba}-\mathrm{Cd}\end{array}$ & $\begin{array}{l}-4 \mathrm{mil} \\
-10 \mathrm{mil} \\
-60 \mathrm{mil}\end{array}$ \\
\hline $\begin{array}{l}\text { White } \\
\text { Sn }\end{array}$ & $\begin{array}{l}-4 \mathrm{mil} \\
-10 \mathrm{mil} \\
-60 \mathrm{mil}\end{array}$ \\
\hline
\end{tabular}

White - $60 \mathrm{mil}$ 


\section{Exposure Sites}

Name and Location "*

Letter Designation for Graphs

Desert Sunshine Exposure Tests, Inc.

A

Phoenix, Arizona

South Florida Test Service, Inc.

F

Miami, Florida

old NBS Site

Connecticut \& Van Ness Streets

W

Washington, D. C.

* Samples were placed on the exposure racks facing south at $45^{\circ}$ from the horizontal. 


\begin{tabular}{c|c}
\hline Property & Company \& Location \\
\hline Color & $\begin{array}{l}\text { American Cyanamid Company } \\
\text { Stamford, Connecticut }\end{array}$ \\
\hline $\begin{array}{c}\text { Tensile } \\
\text { Flexure }\end{array}$ & $\begin{array}{l}\text { W. R. Grace \& Company } \\
\text { Clarksille, Maryland }\end{array}$ \\
\hline Haze & $\begin{array}{l}\text { Monsanto Company } \\
\text { Indian Orchard, Massachusetts }\end{array}$ \\
\hline Gloss & $\begin{array}{l}\text { Hercules Incorporated } \\
\text { Wilmington, Delaware }\end{array}$ \\
\hline
\end{tabular}




\section{List of Figures}

Numbers

$1-20$

Color-change, $\triangle \mathrm{E}$ (De1ta $\mathrm{E})$

$21-40$

Ultimate Elongation (\% of Initial Value)

$41-48$

5 Percent Stress (PSI)

$49-68$

Gloss (in percent)

$69 A-81 A$

Haze at $421 \mathrm{~nm}$ (in percent)

$69 B-81 B$

Haze at $550 \mathrm{~nm}$ (in percent) 
EI CURE 1

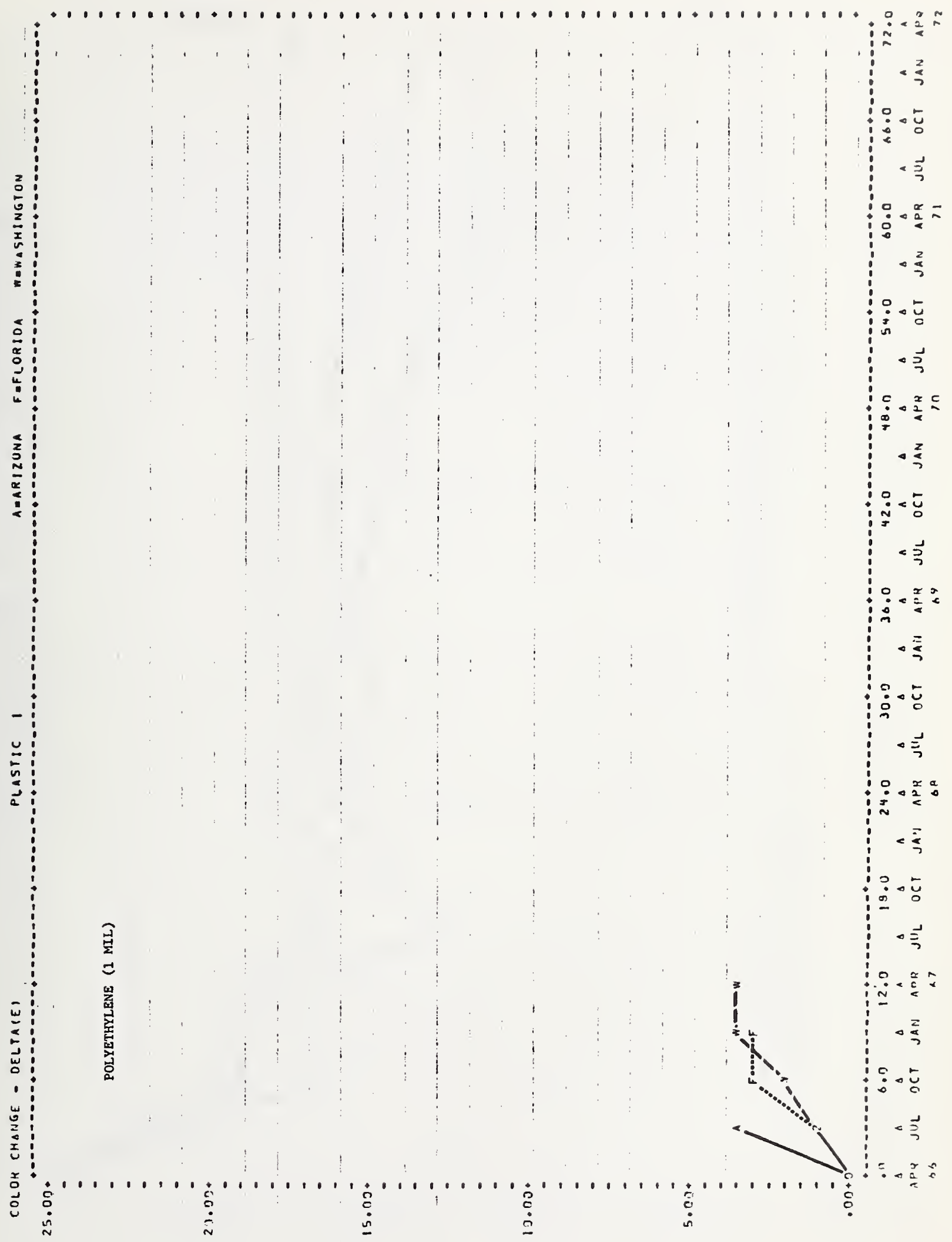


FIGURE 2

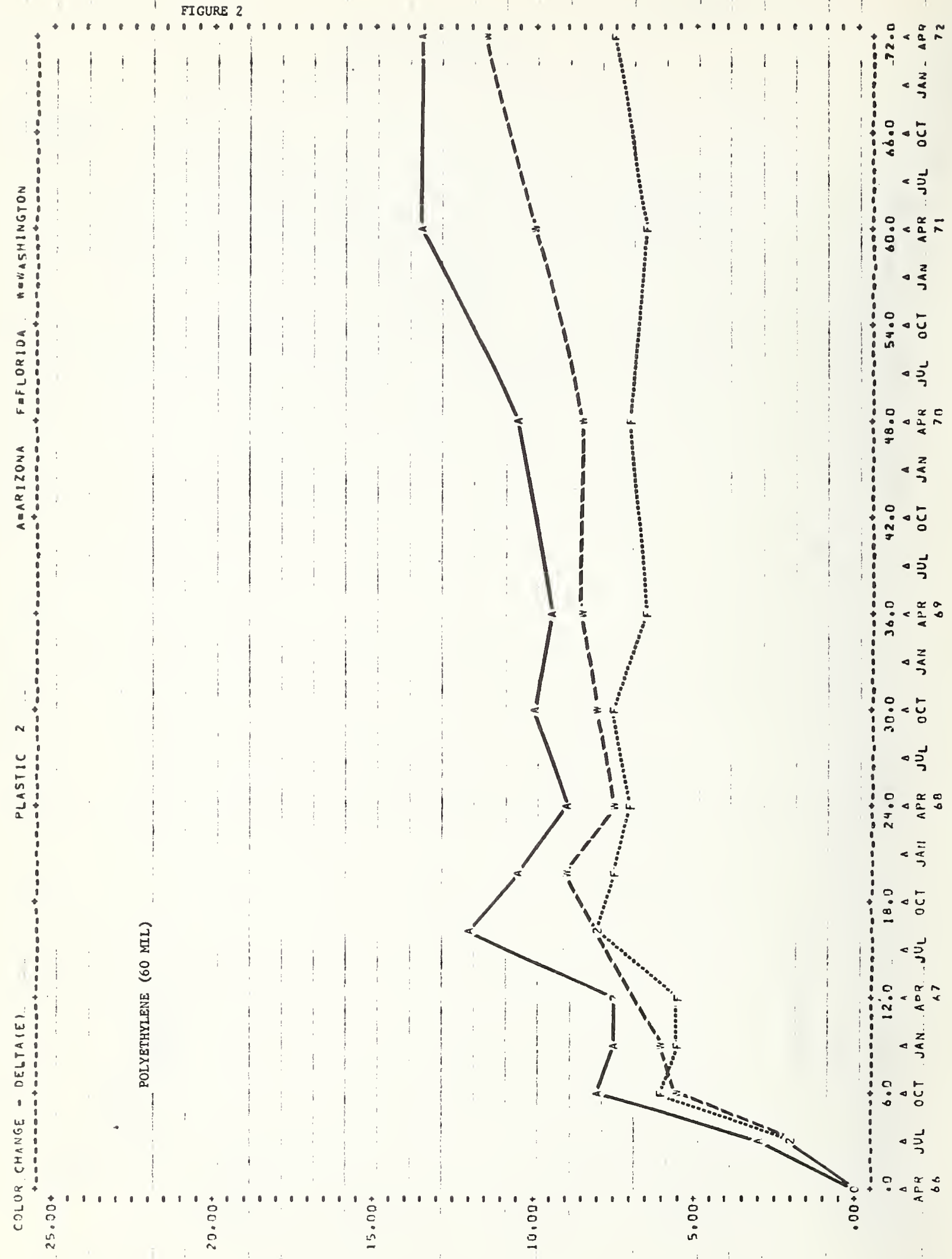


PICURE 3

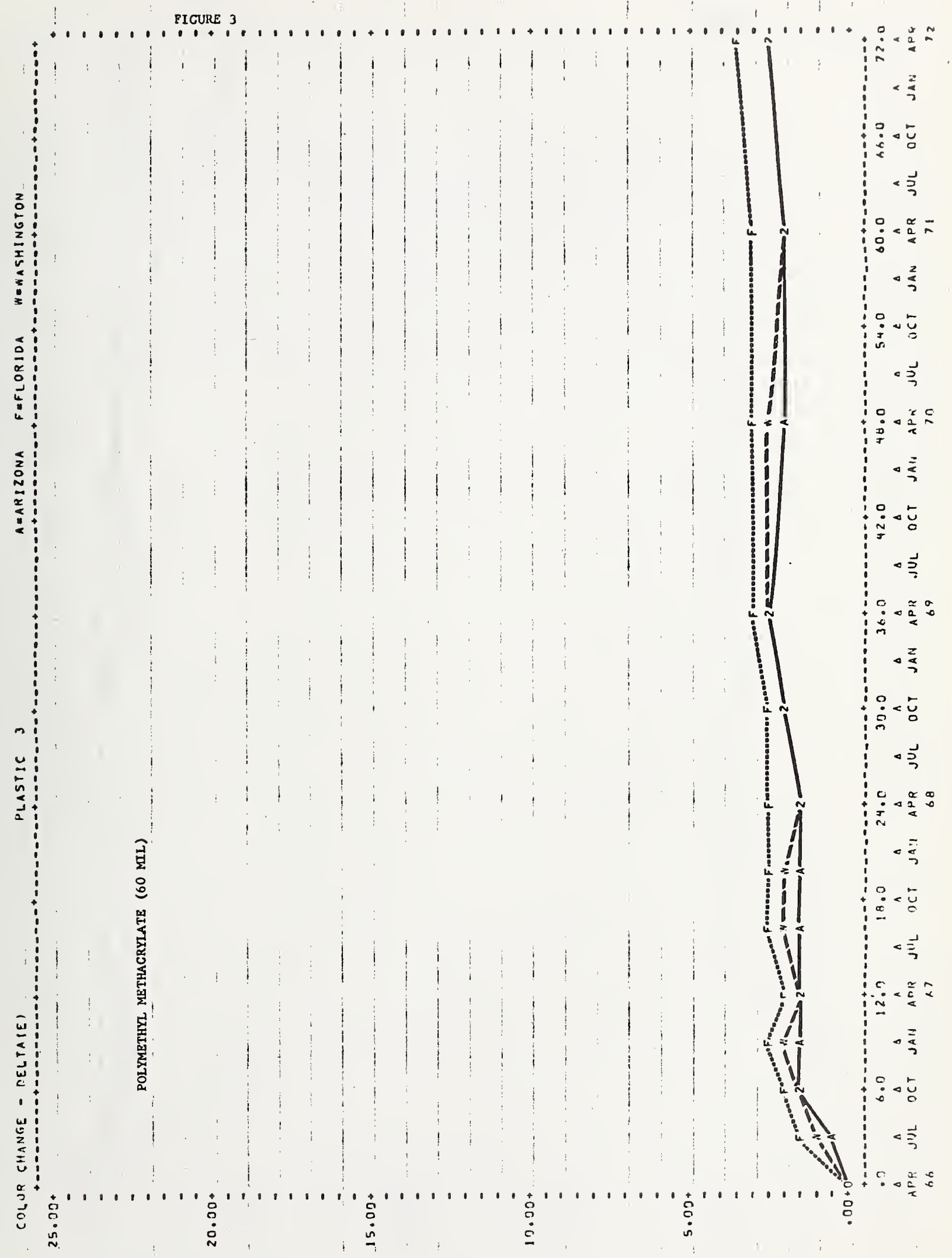


PIGURE 4

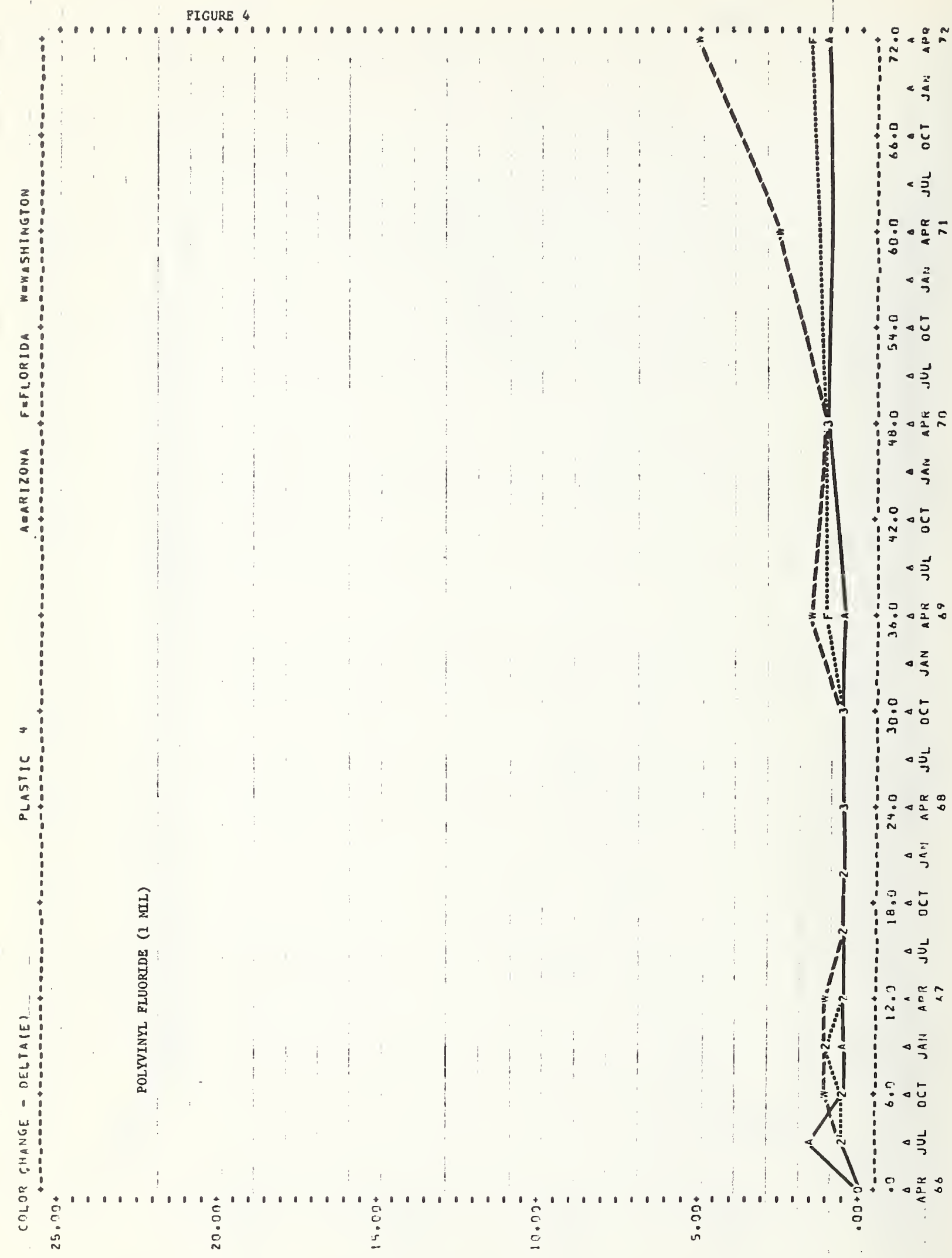


PIGURE 5

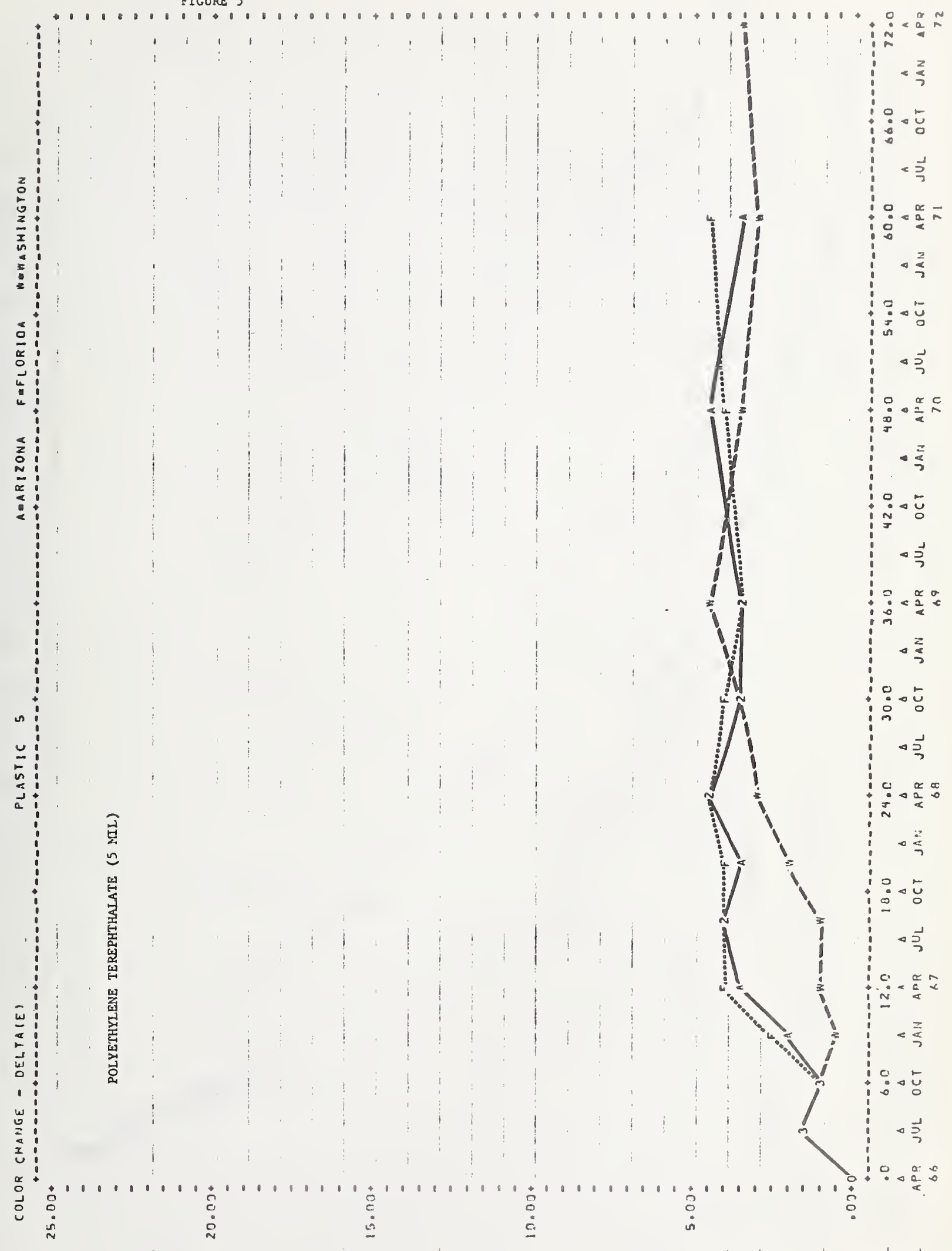


BIGURE 6

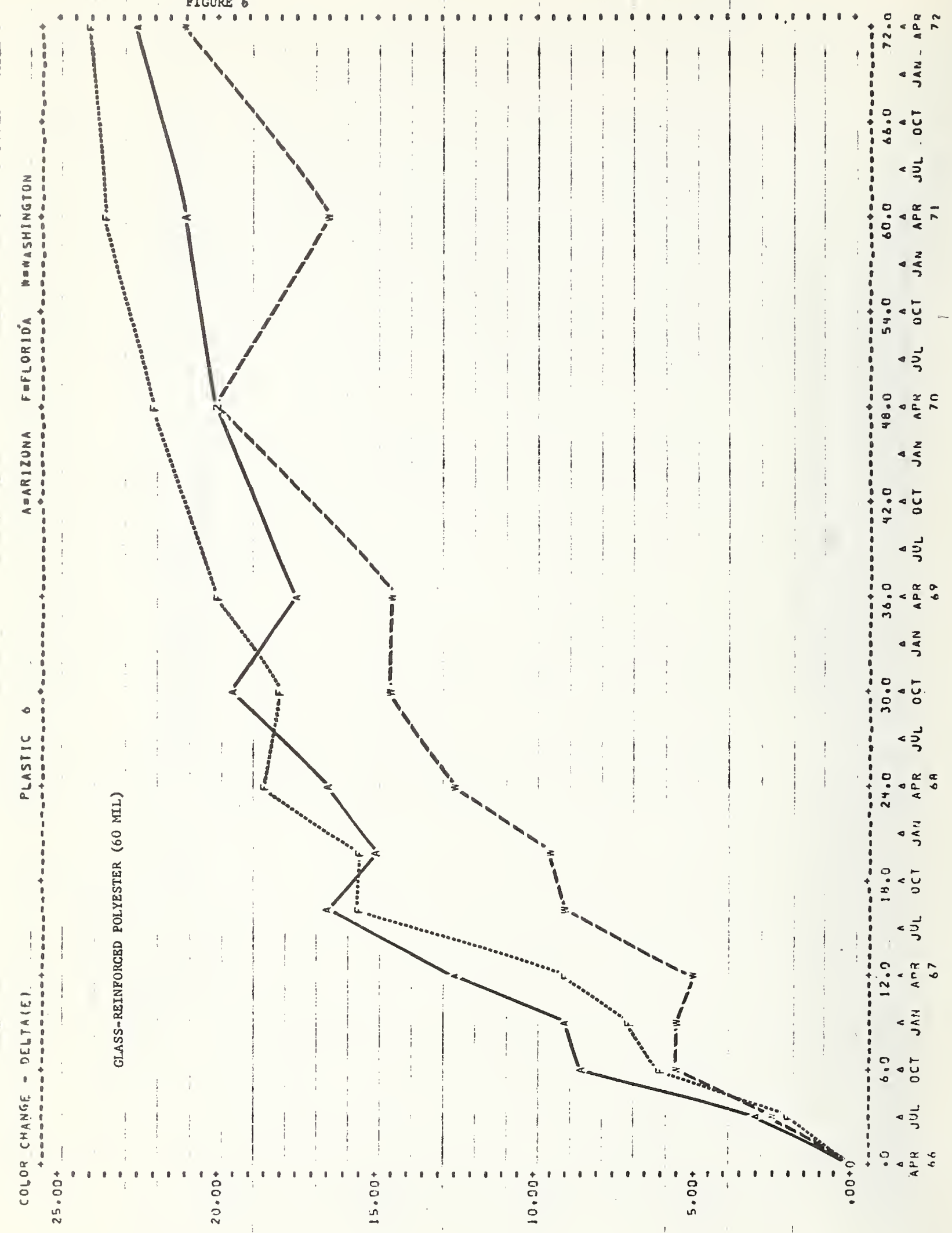


FIGURE 7

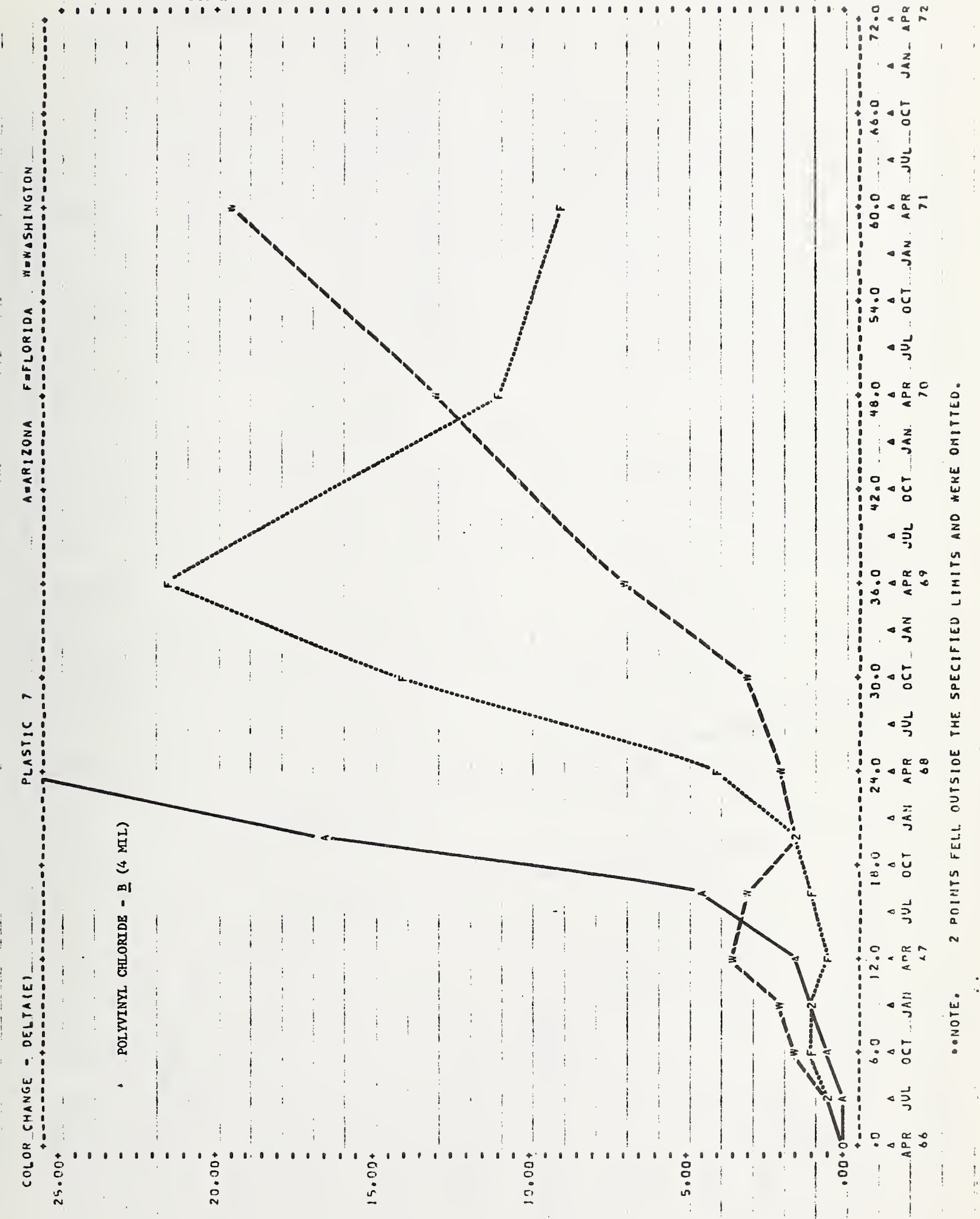




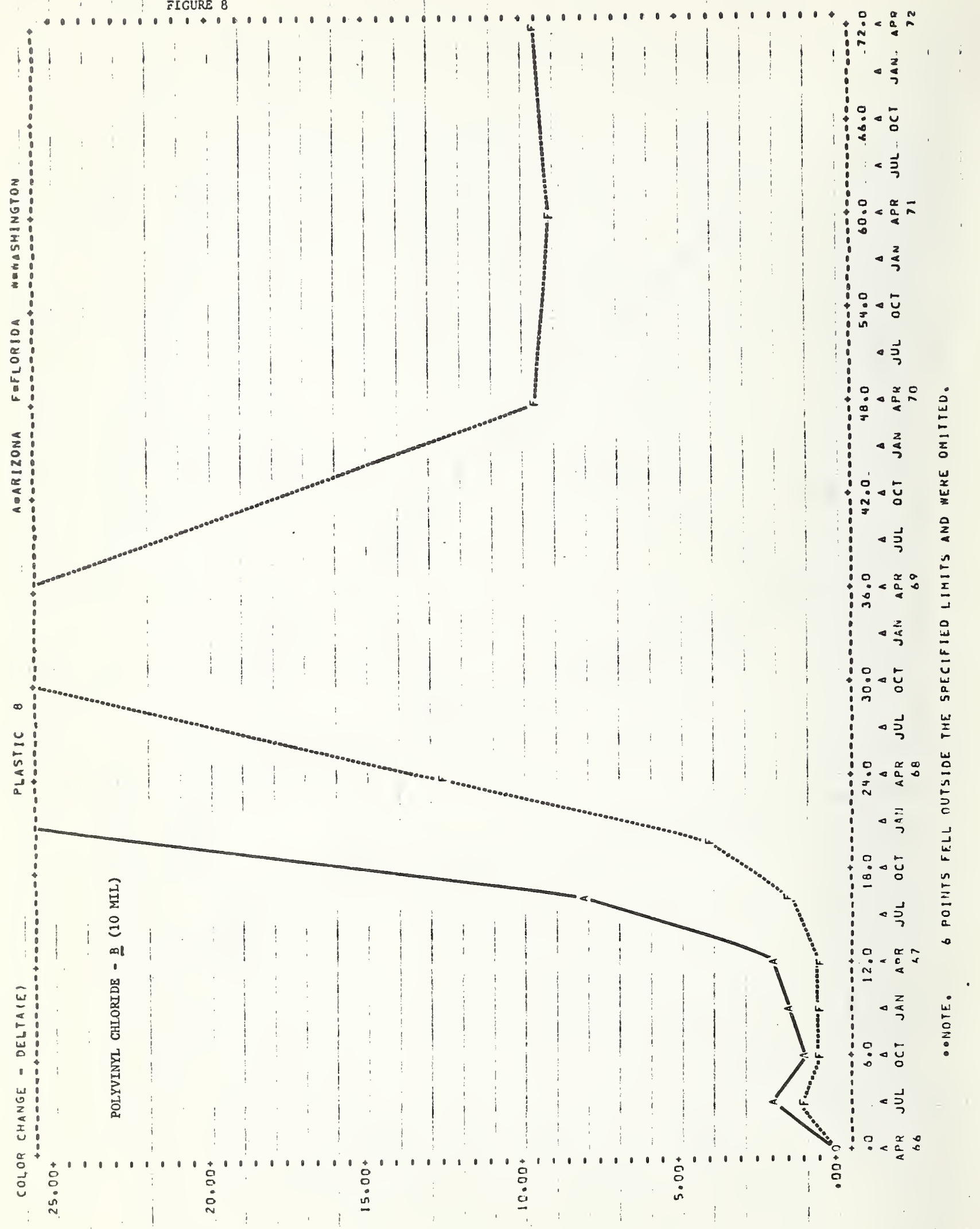


FIGURE 9

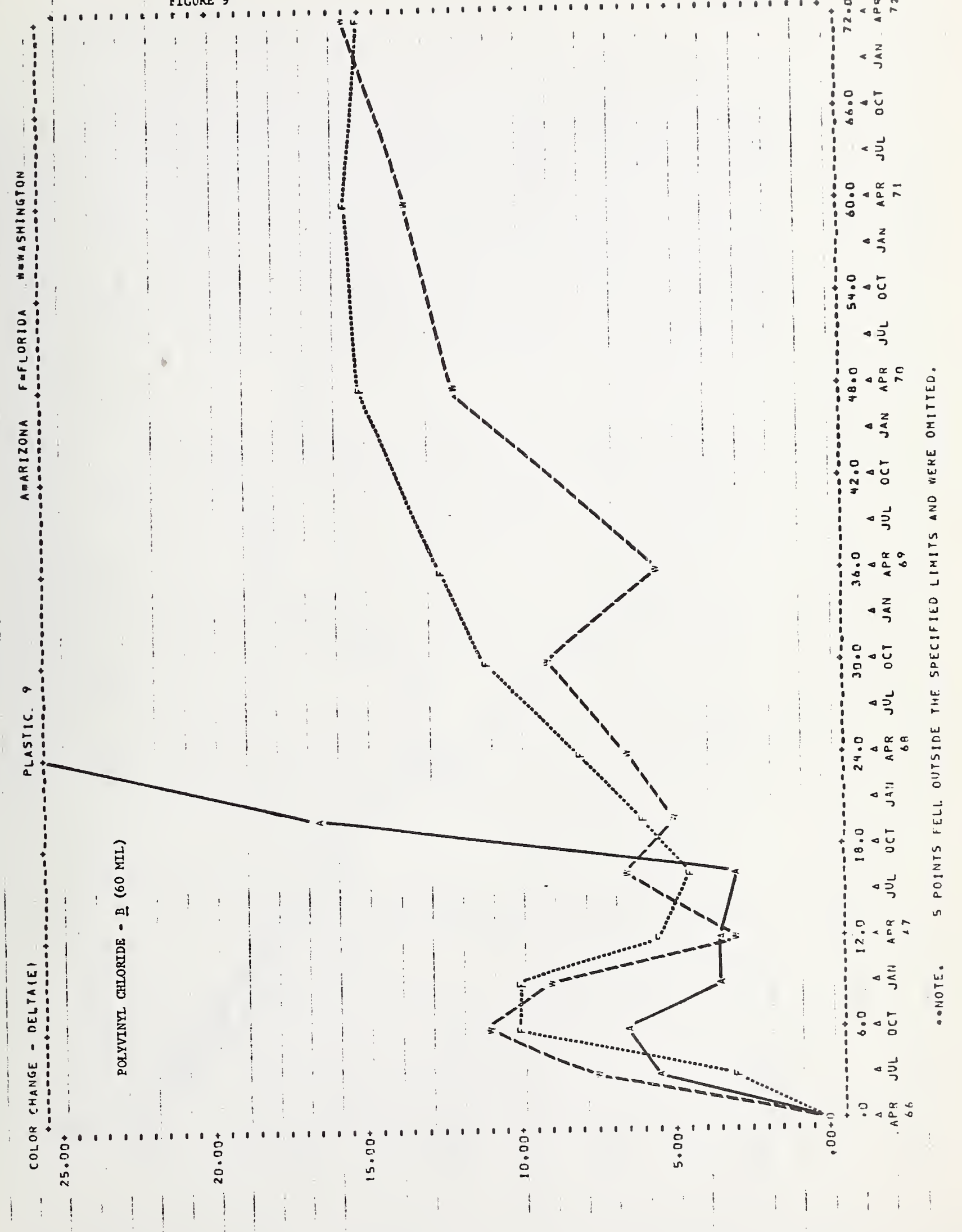


FICURE 10

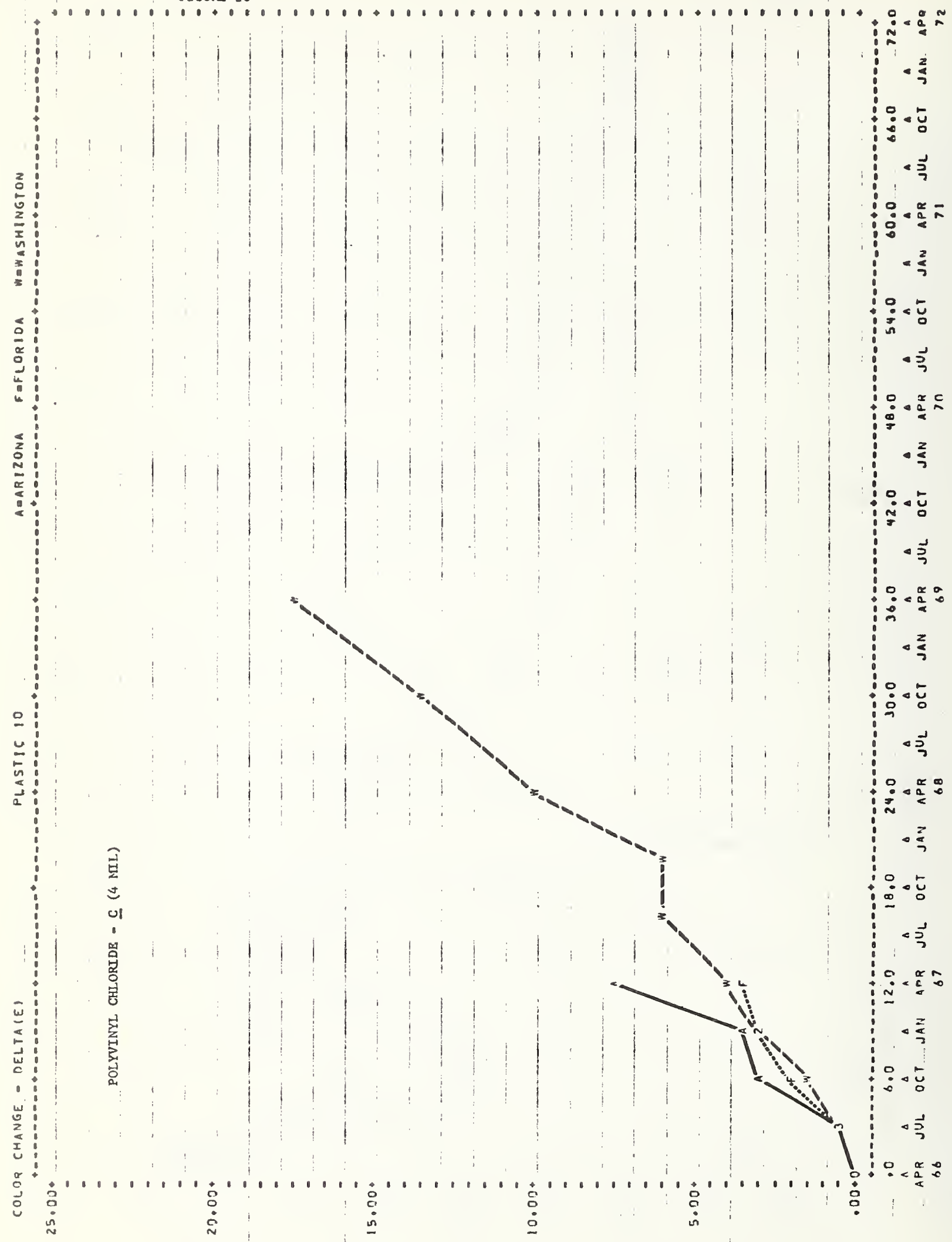


FIGURB 11

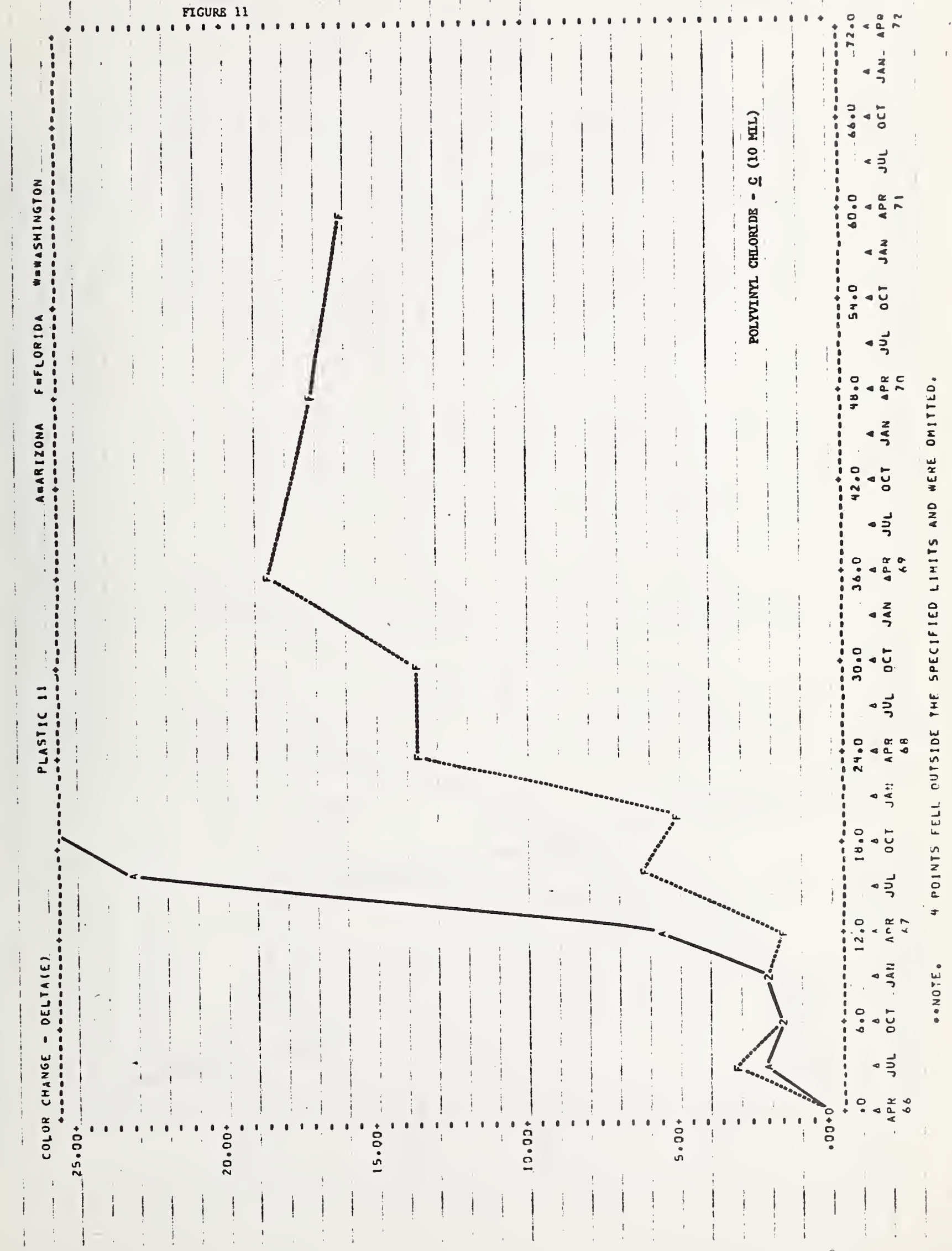


FIGURE 12
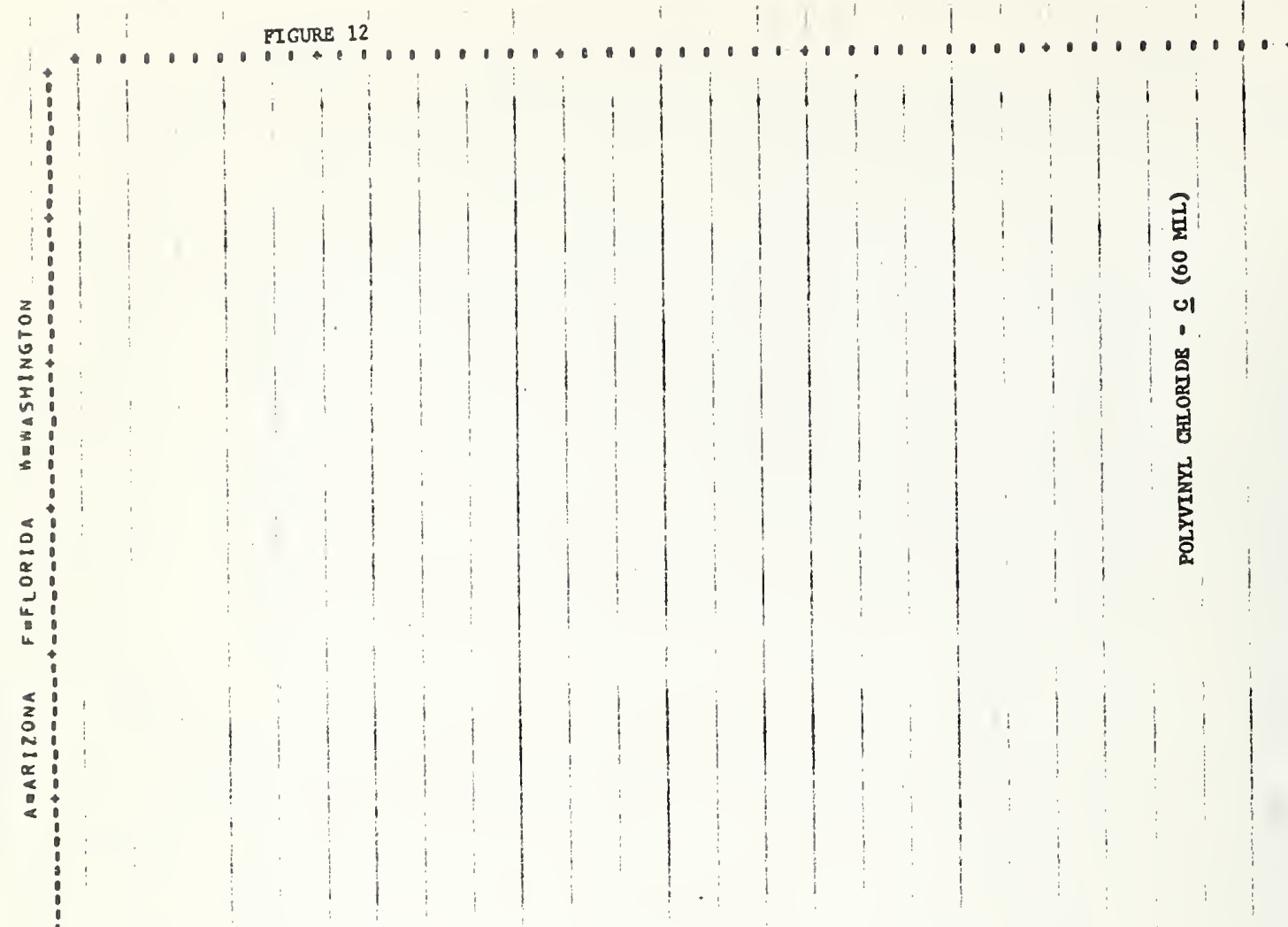

$i \dot{i}<\frac{a}{a}$

$-\frac{3}{2}$

$: \because-\frac{1}{0}$

<국

$: \because \frac{\alpha}{2}=$

- $\frac{2}{2}$

$\because \div$

-3

$: 0 \frac{\alpha}{2}$

- 点

i $\overline{0}$

:

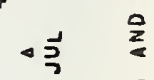

$0 \frac{x}{2}: 5$

- z

$\ddot{0}-5$

$\simeq$

垔

㖕 崖

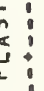

1

w

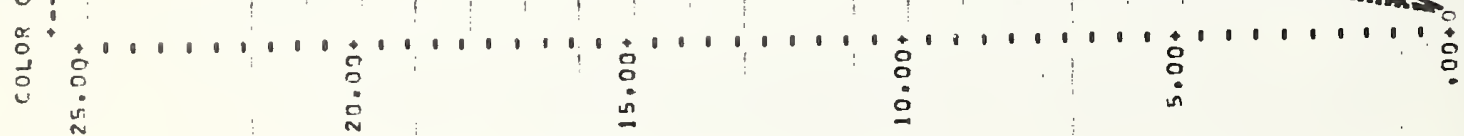

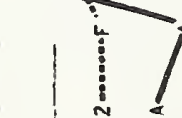

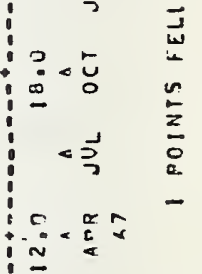

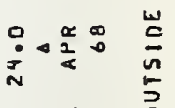

$<\frac{7}{2} \quad$

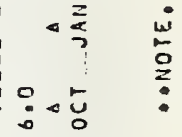

- 고

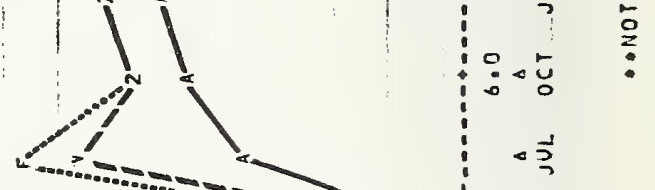


FIGURE 13

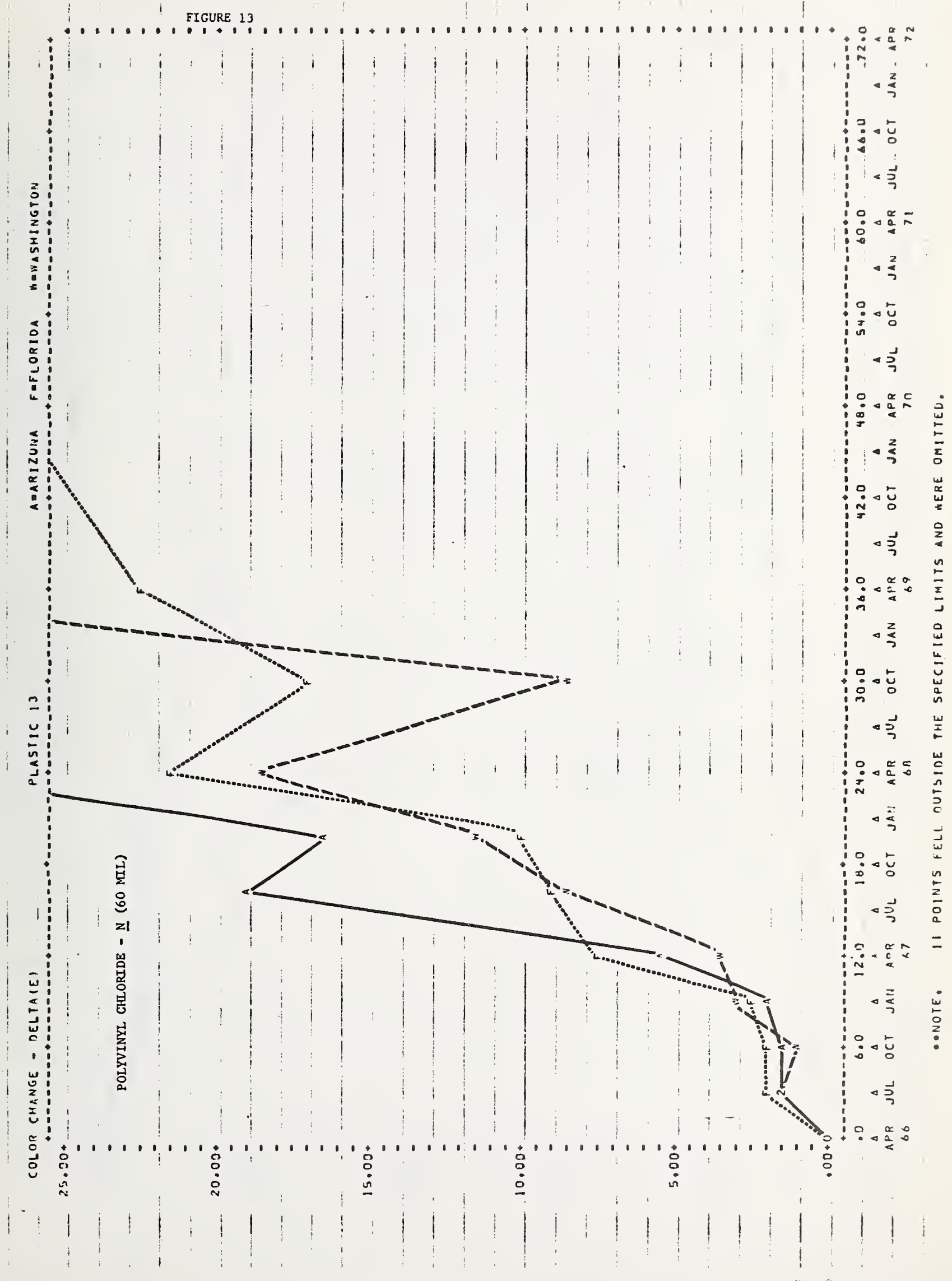


FICURE 14

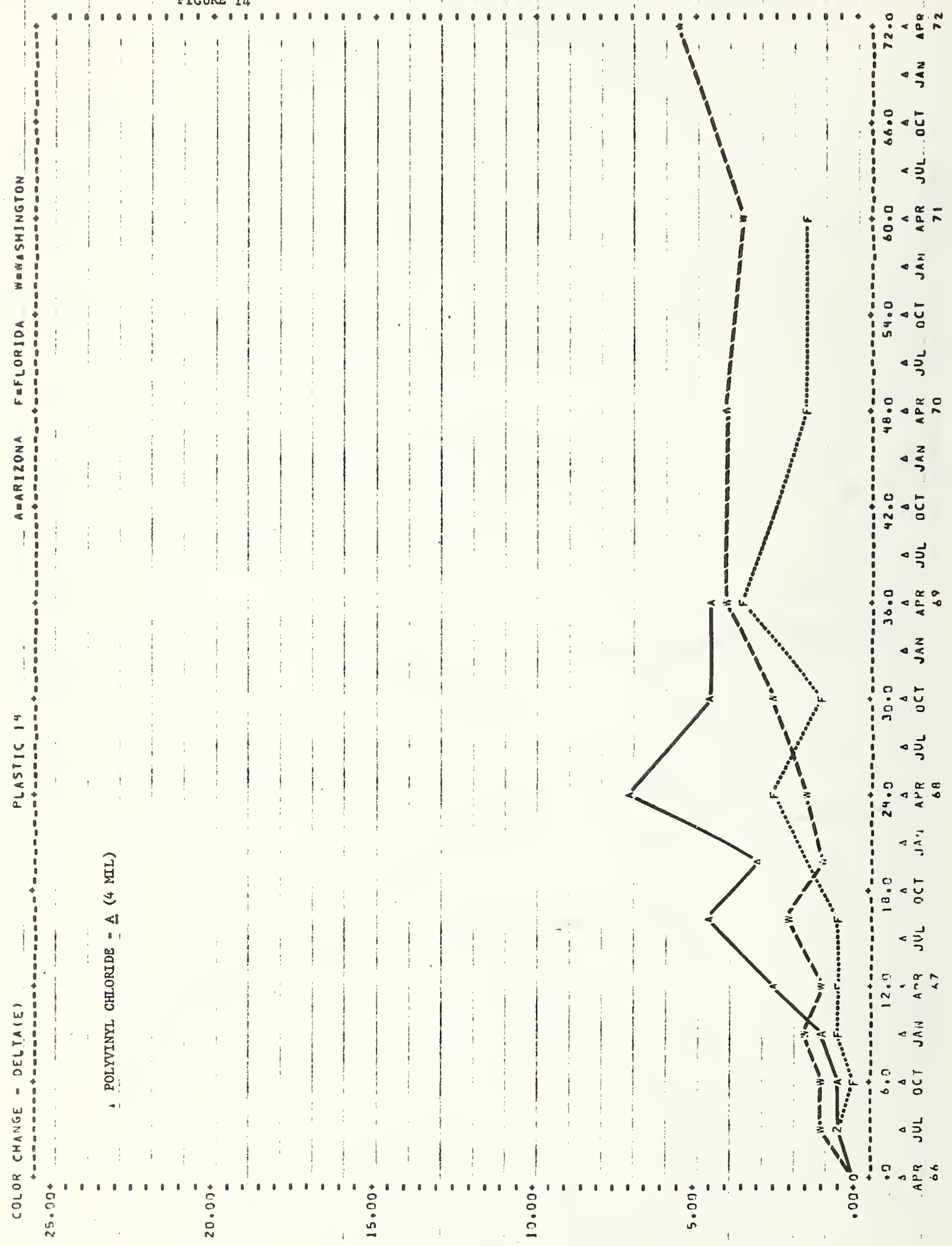


FIGURE 15

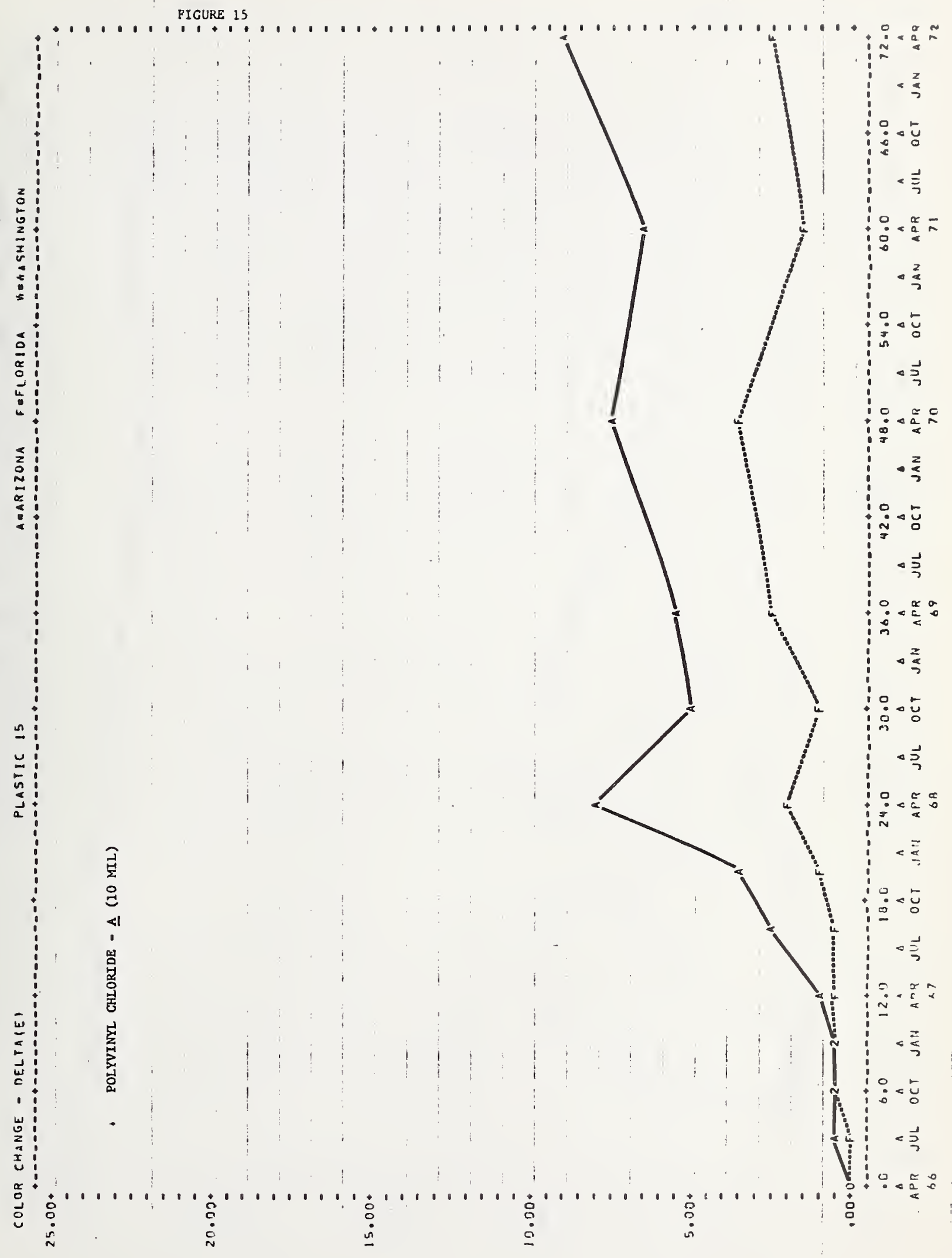




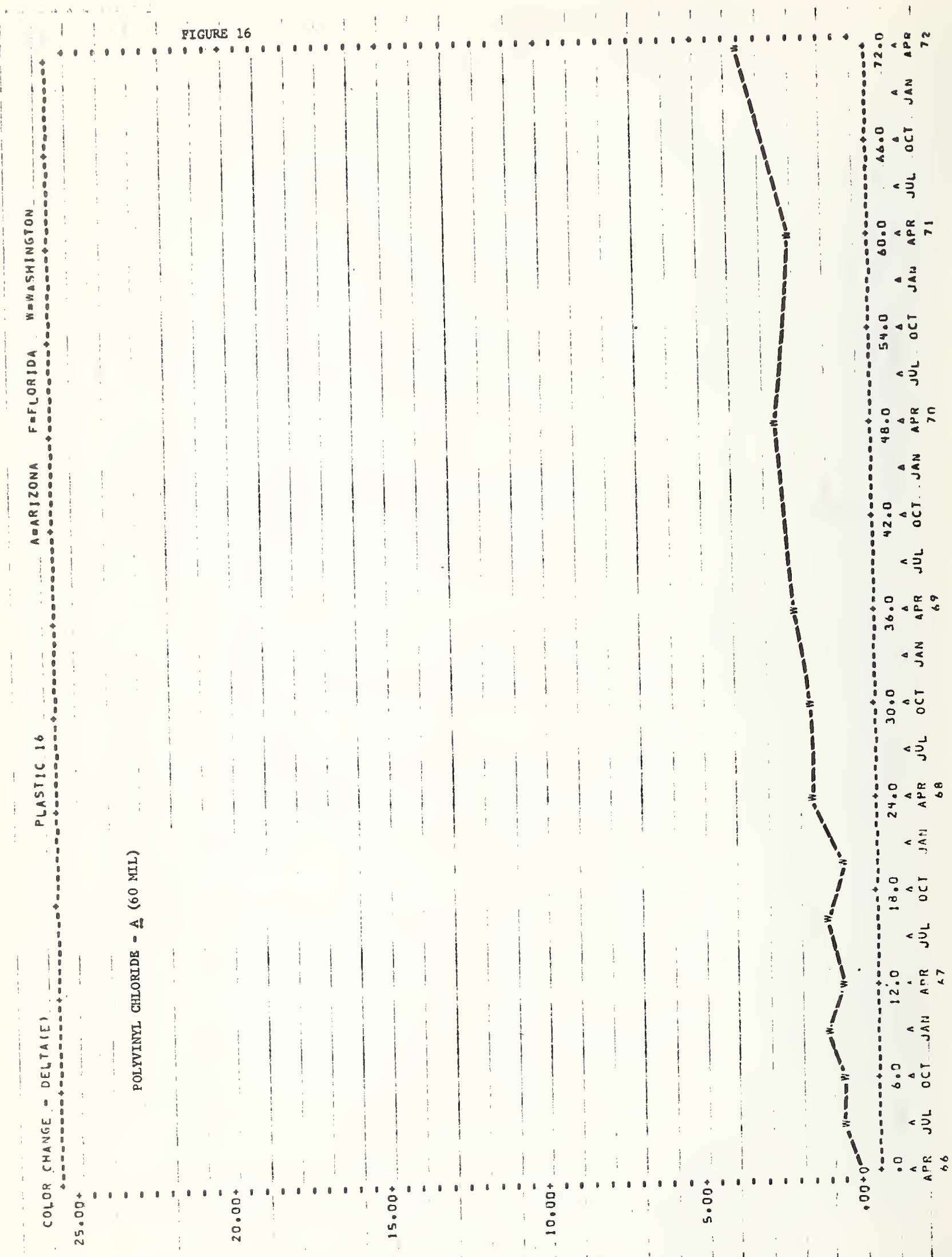


RIGURE 17

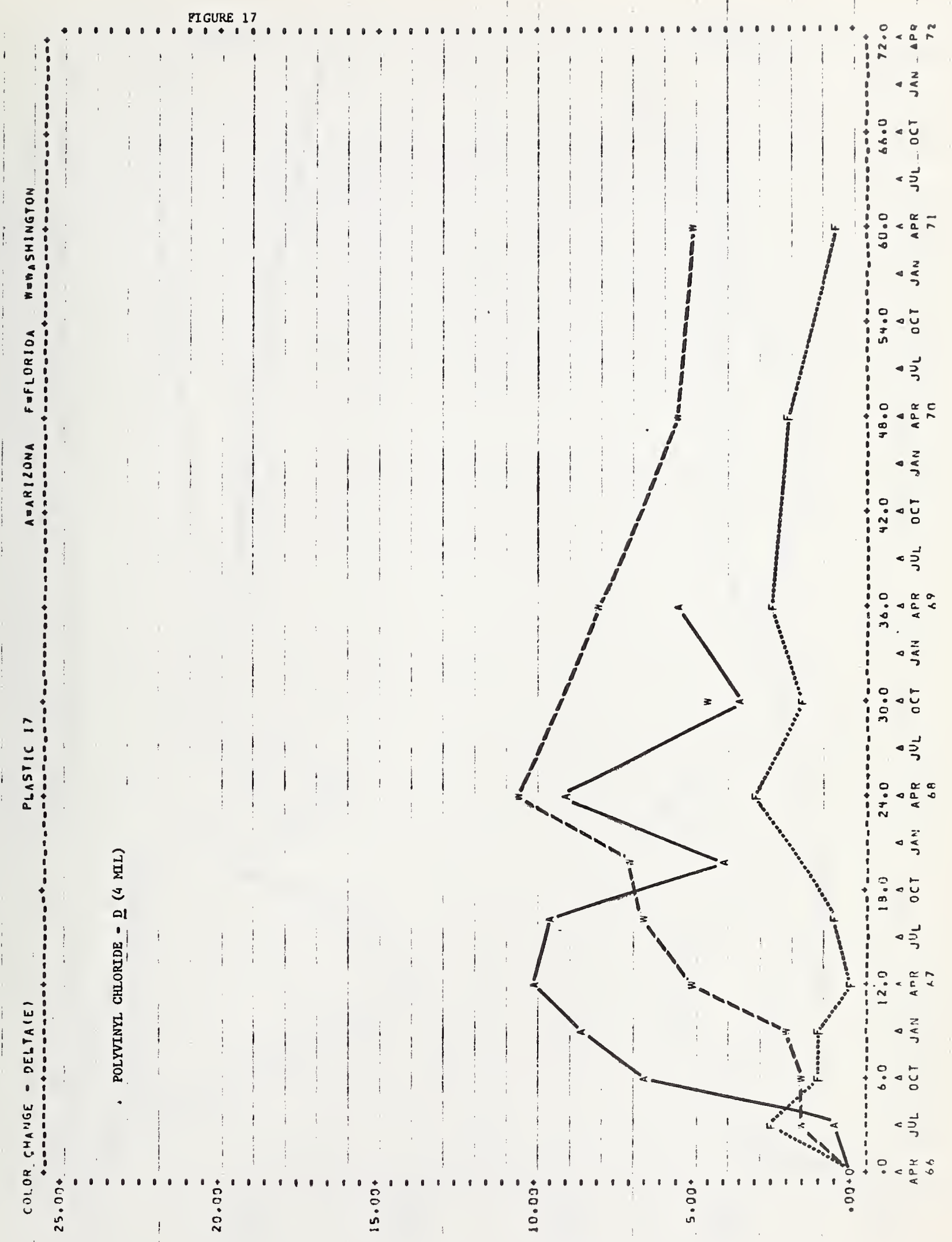


FIGURE 18

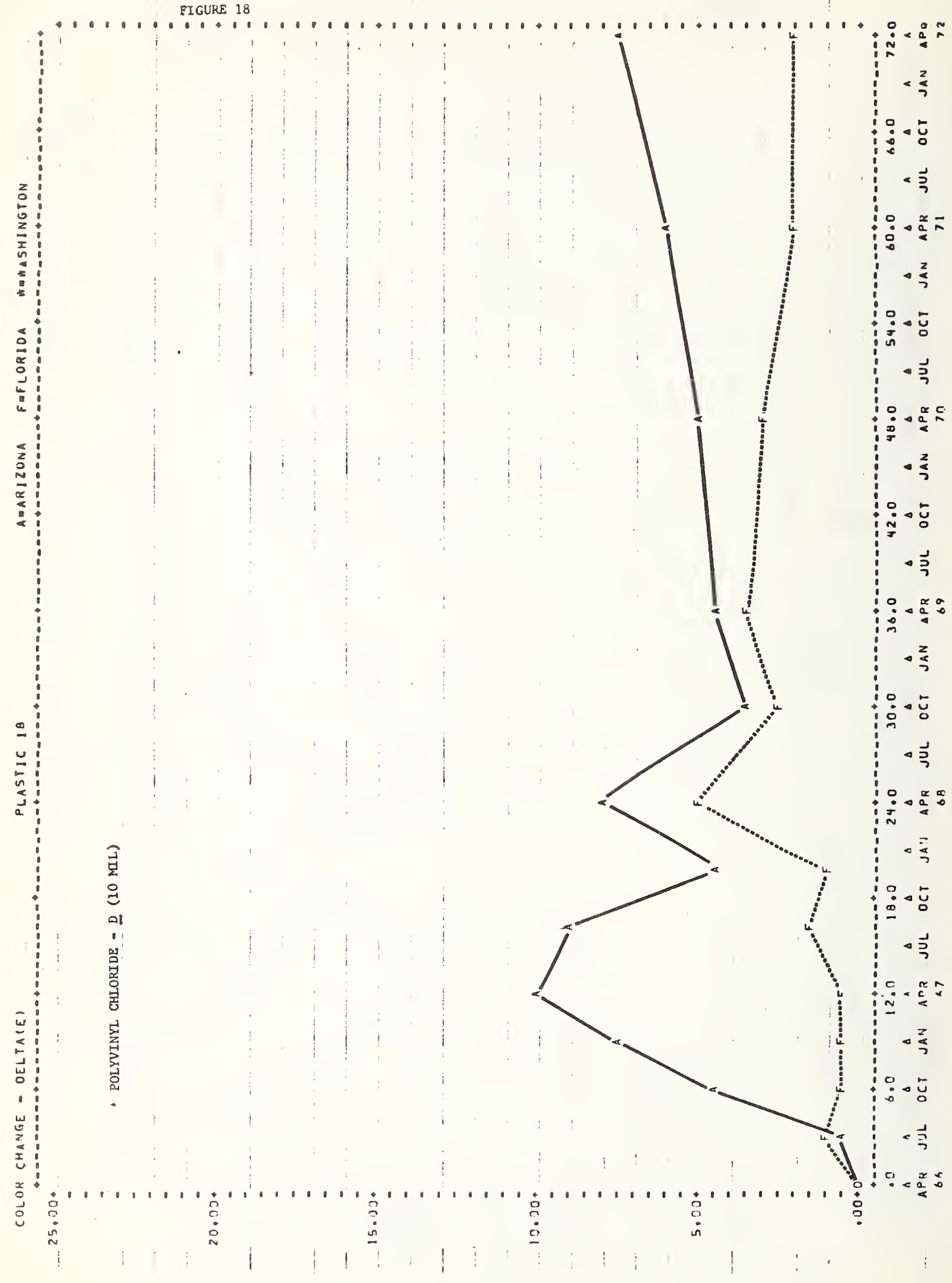


FIGURE 19

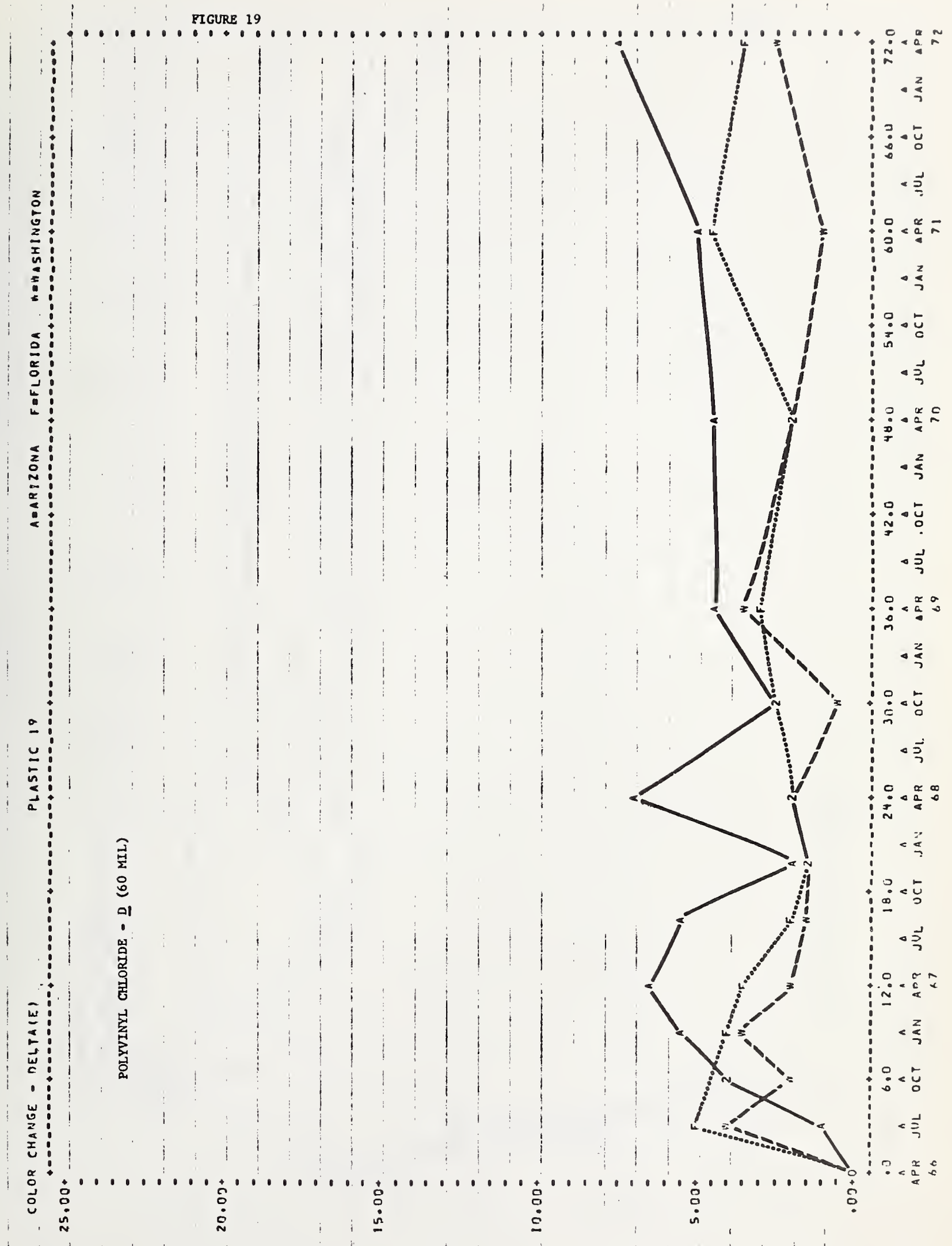




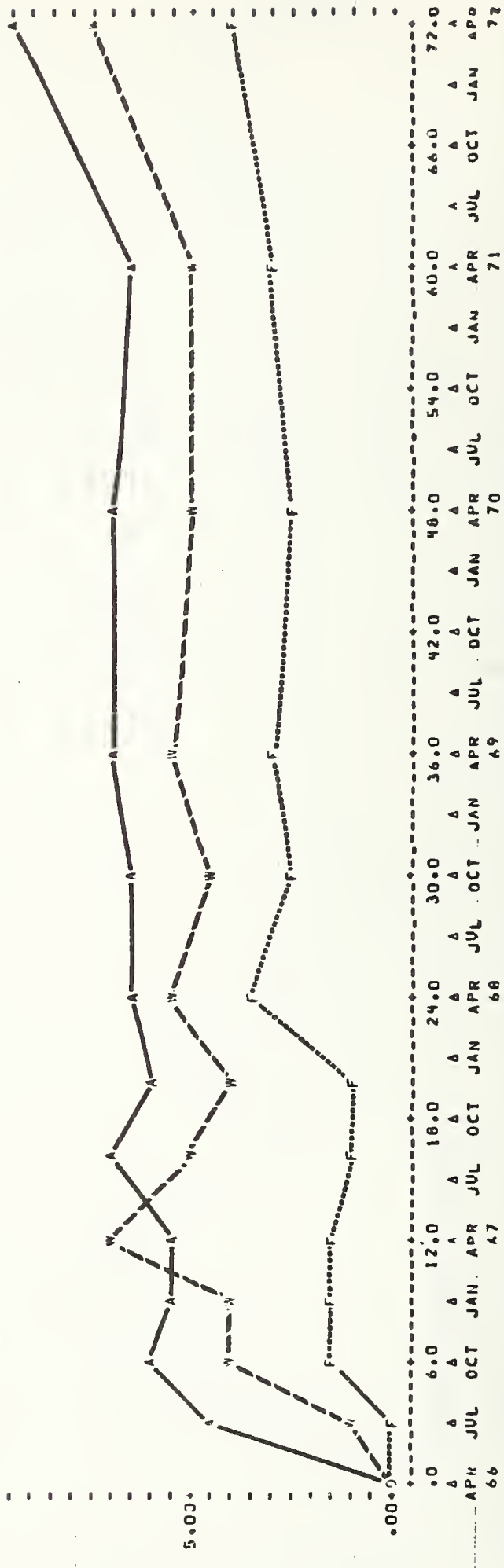


FIGURE 21

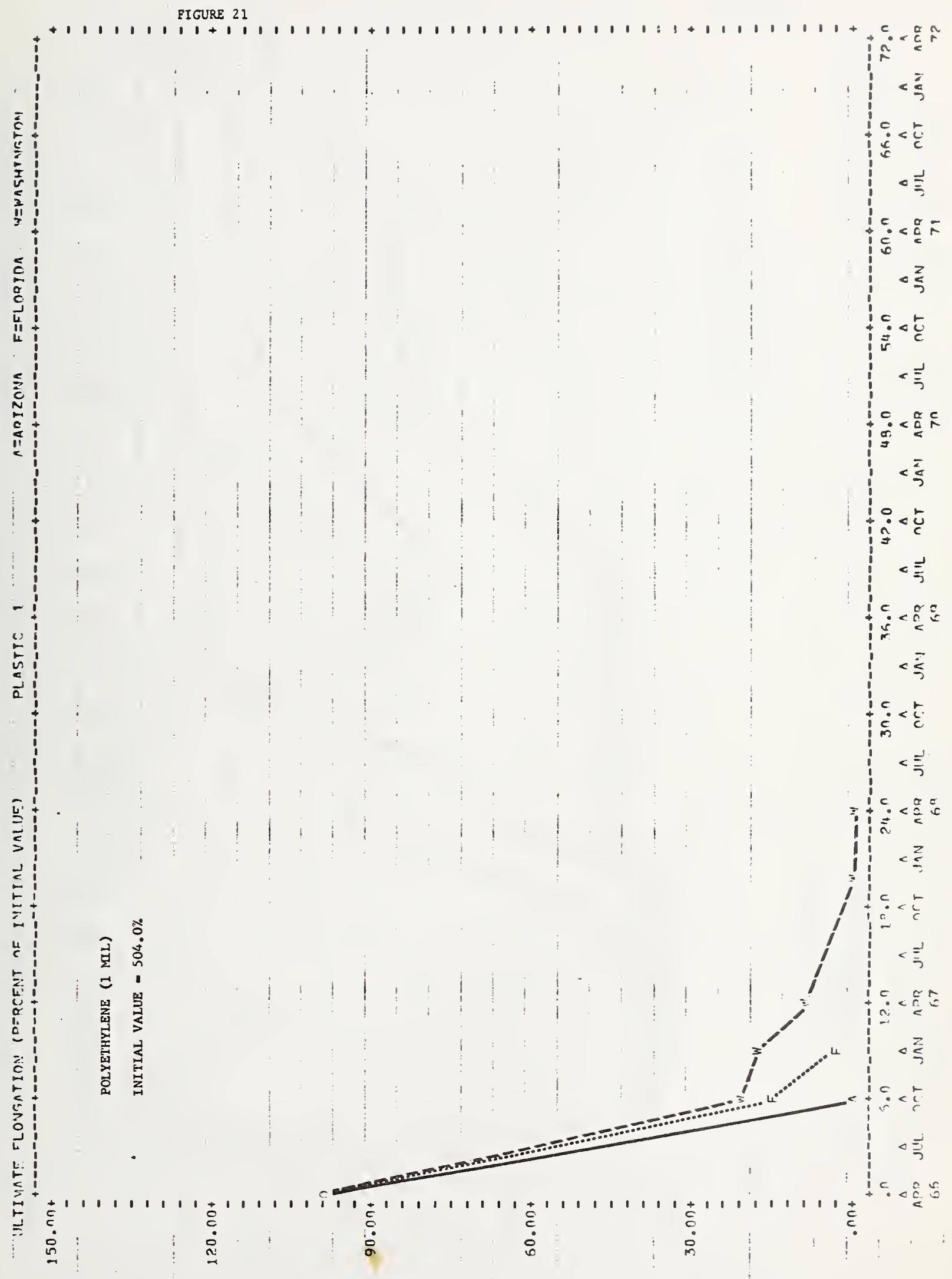


PI GUKR 22

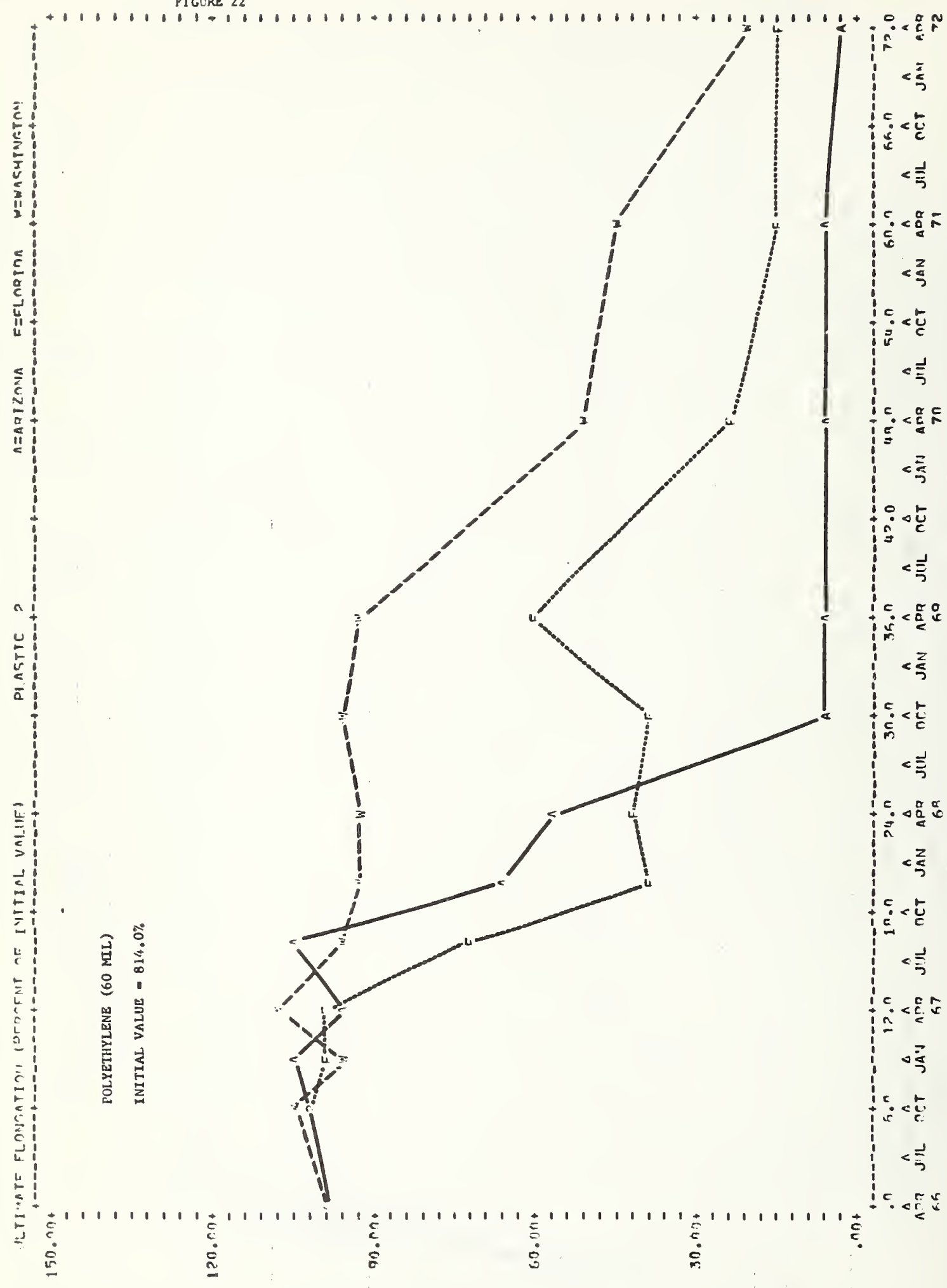




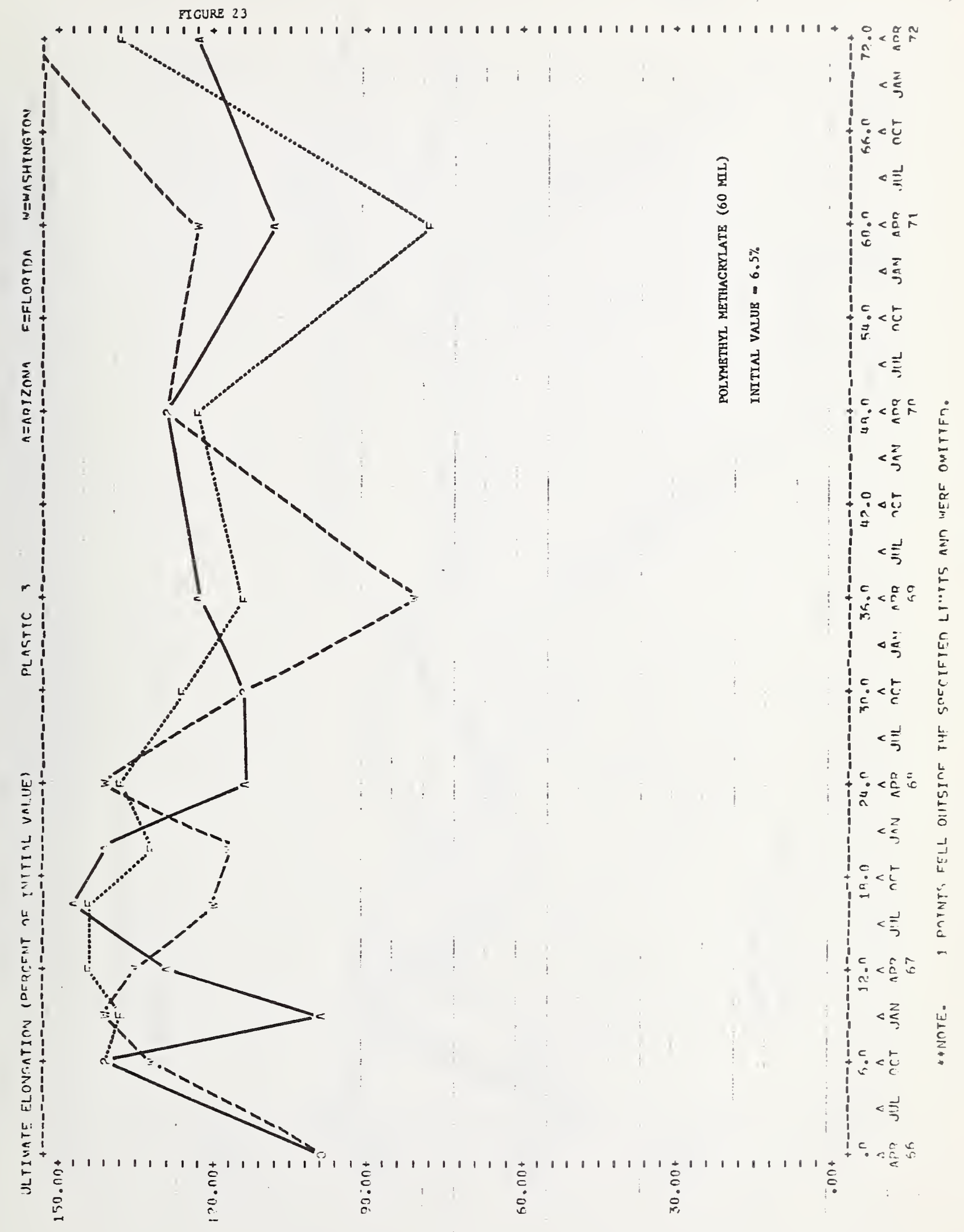




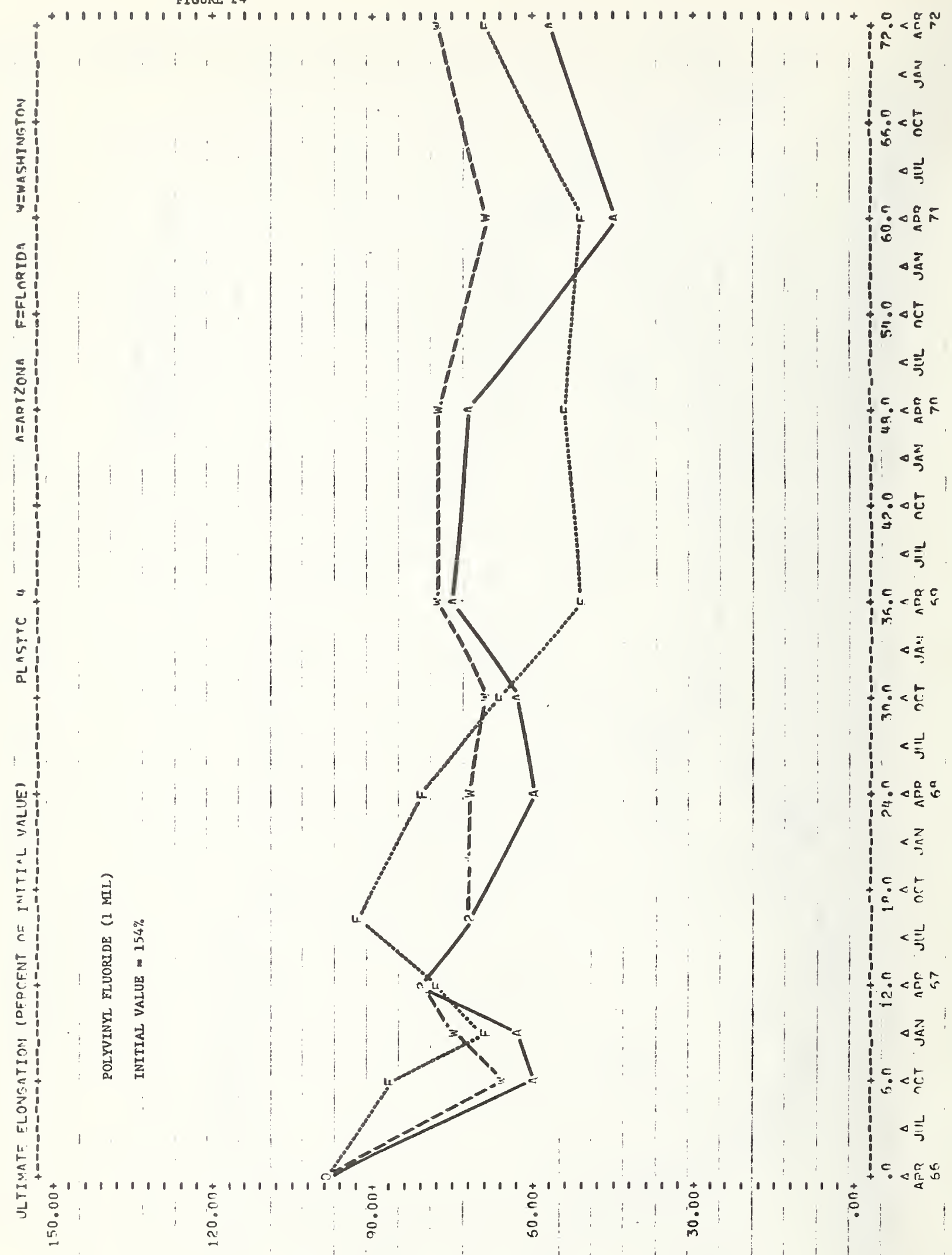


FIGUKE 25

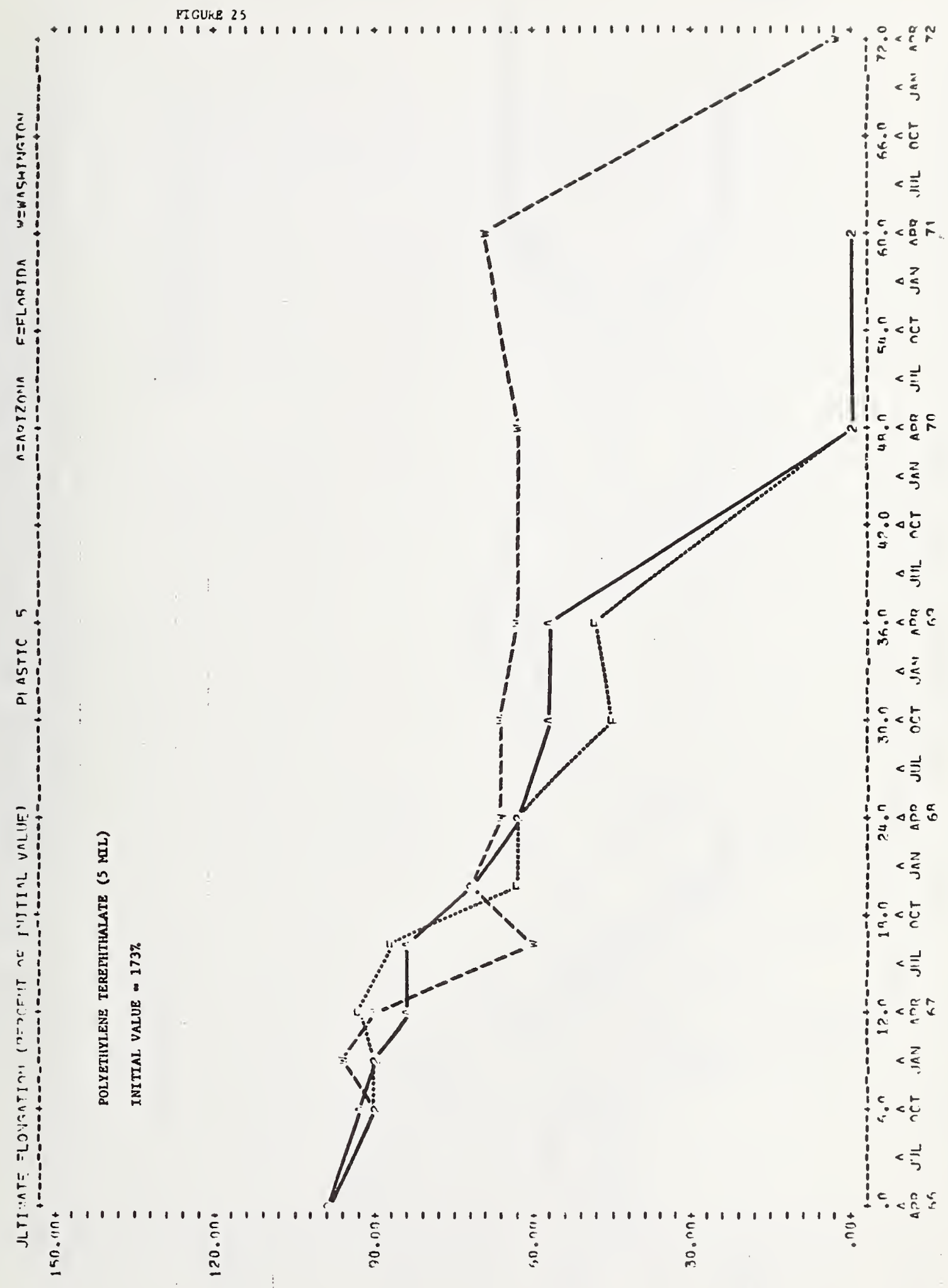




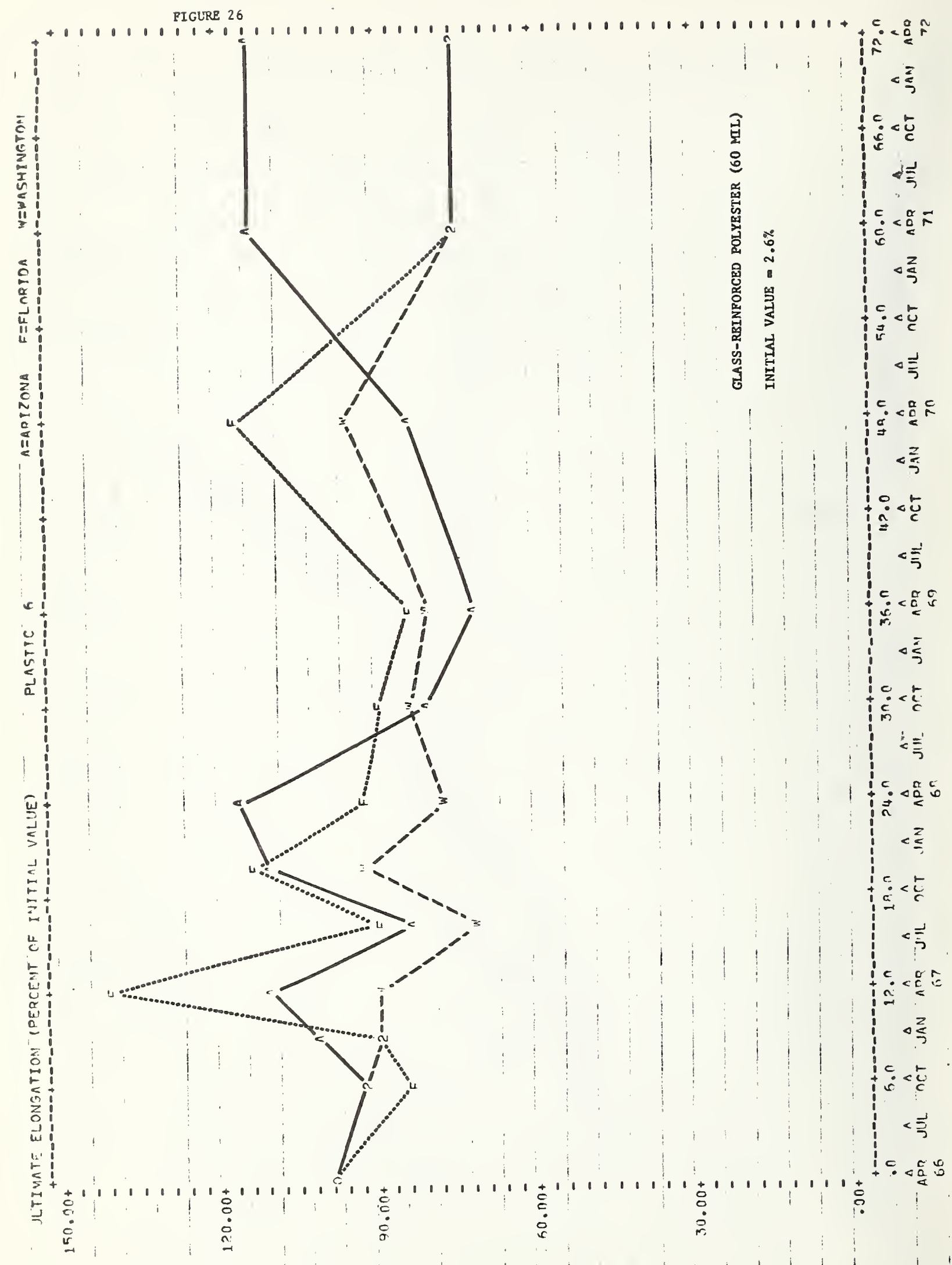


PIGURE 27

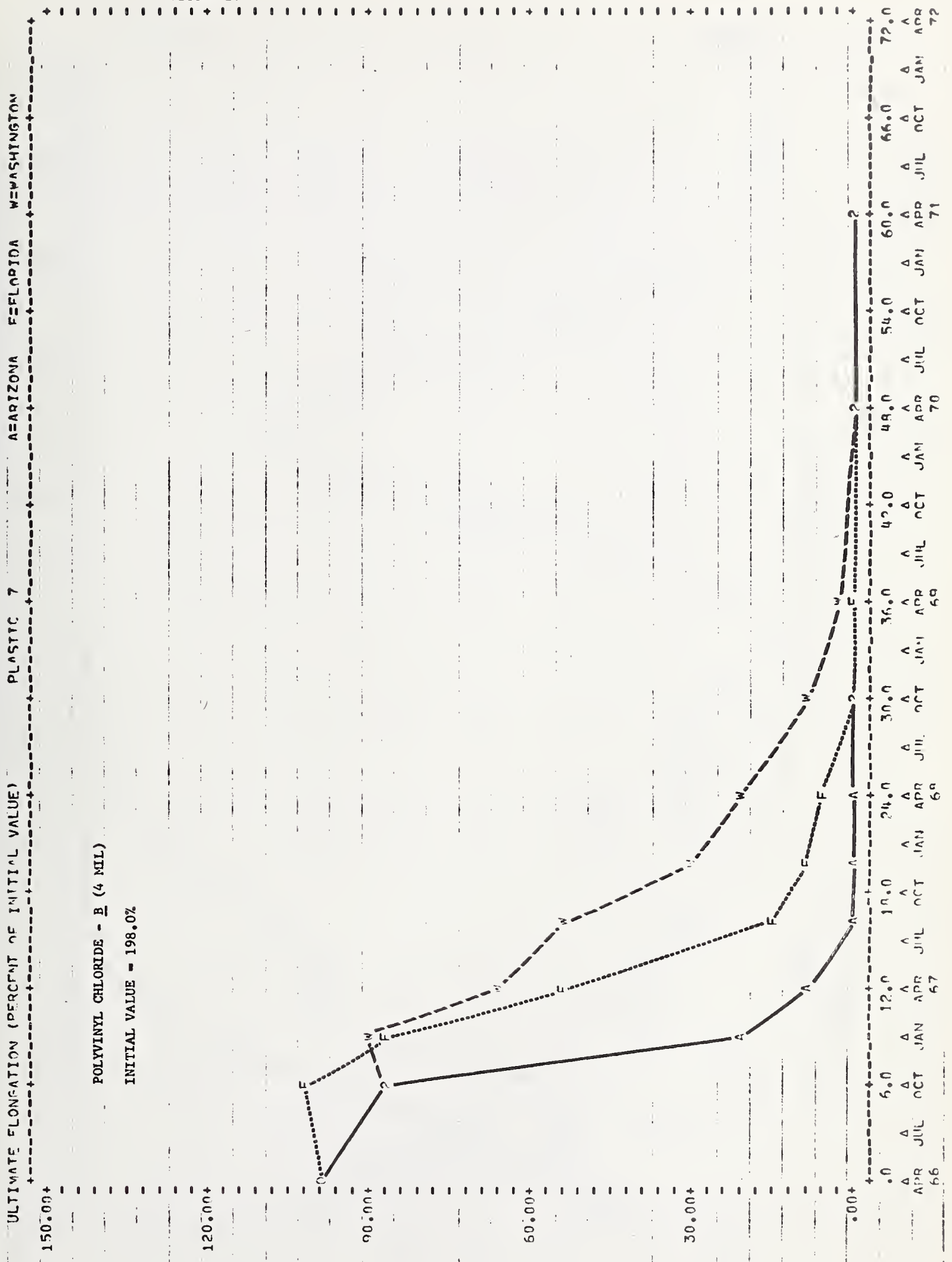




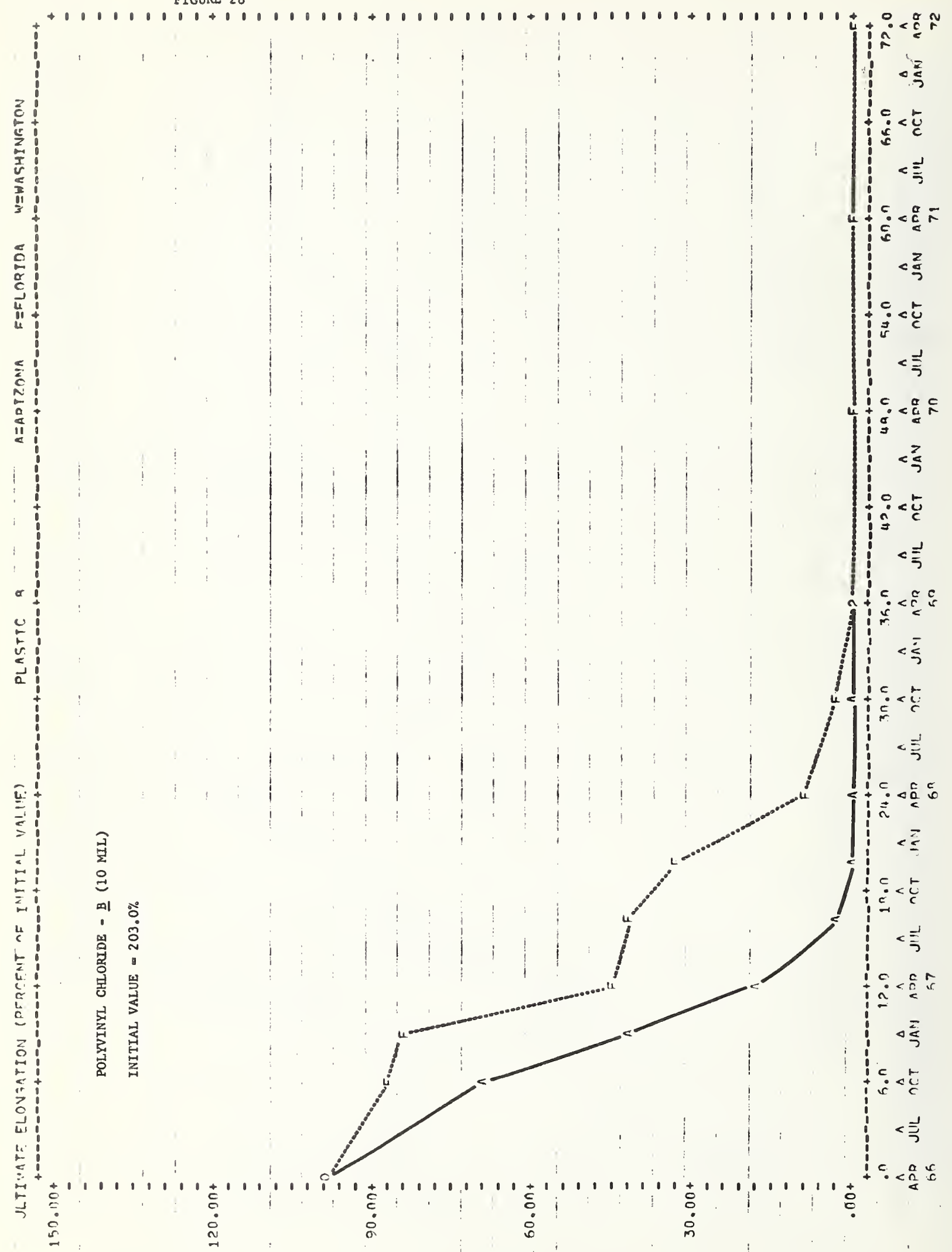


FIGURE 29

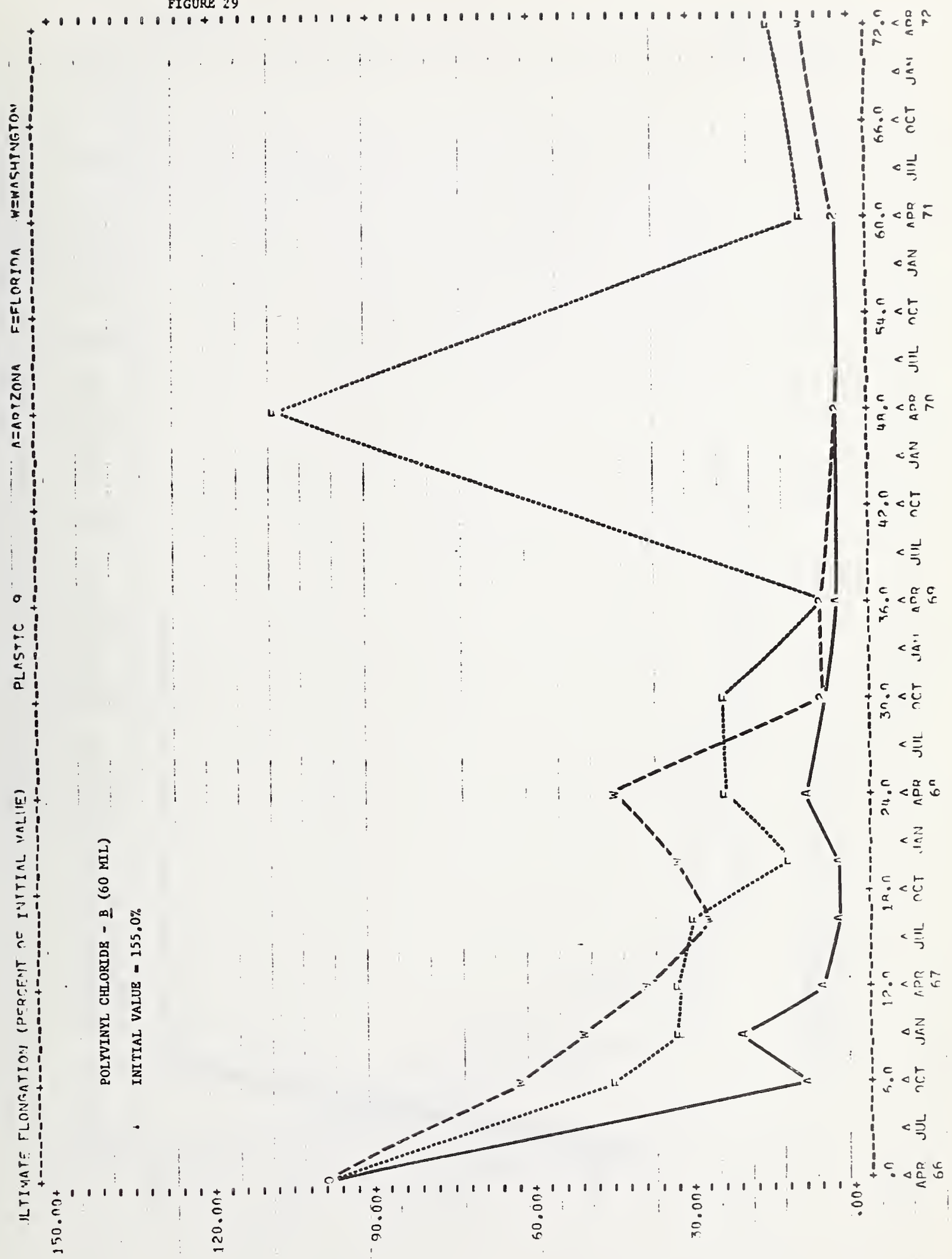


YIGUKE 30

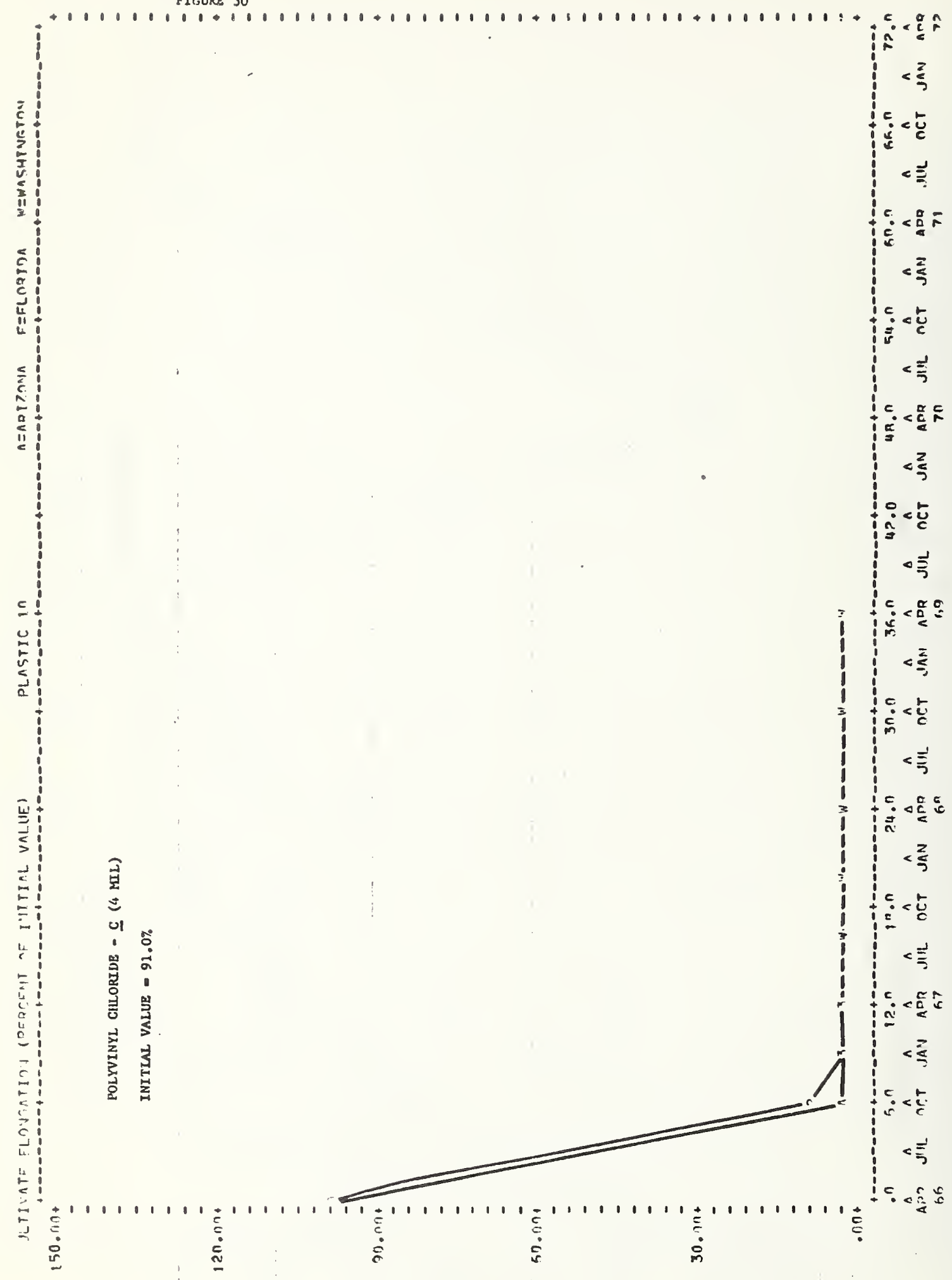


PIGUKE 31

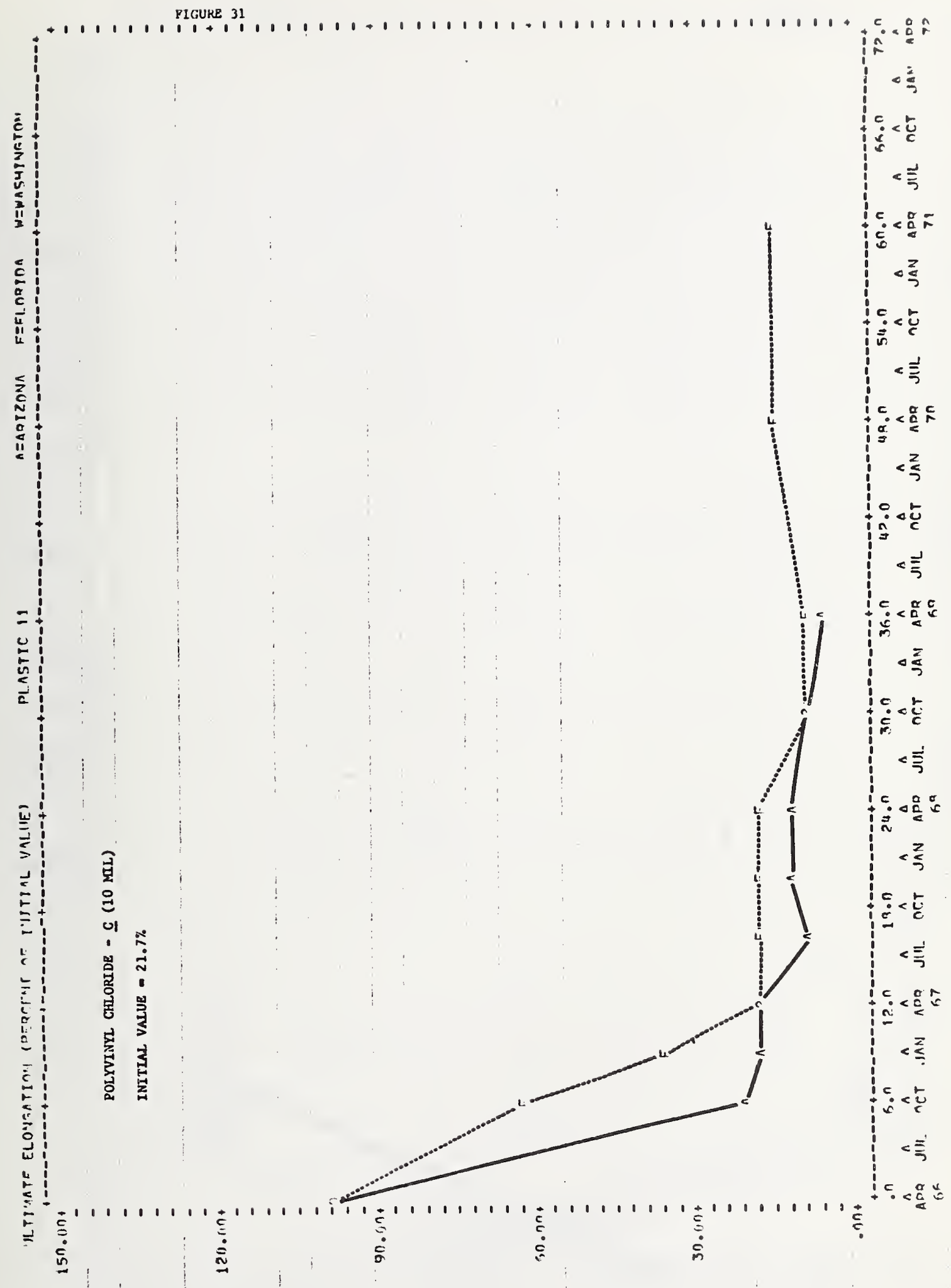


FIGURE 32

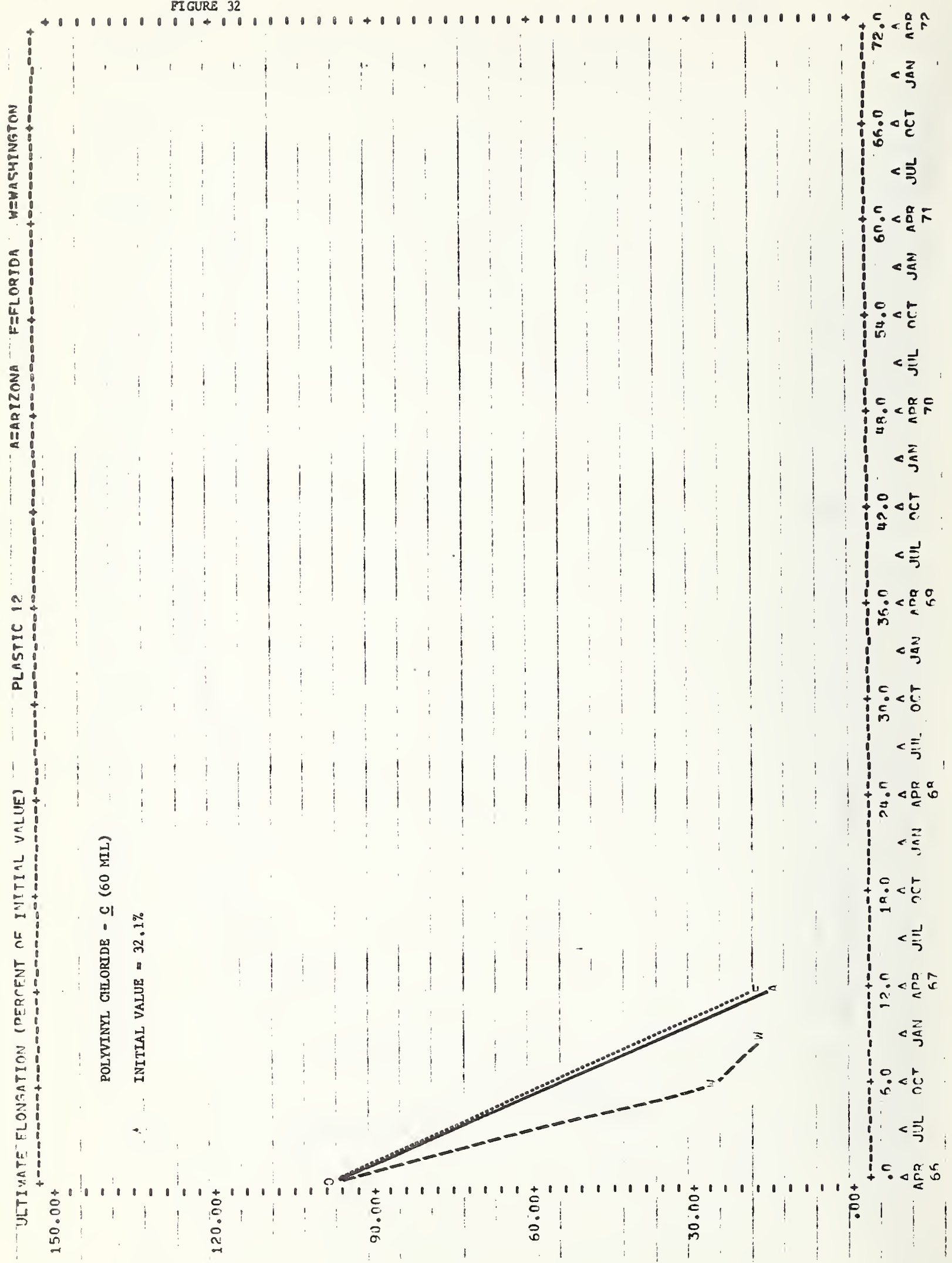


FICURE 33

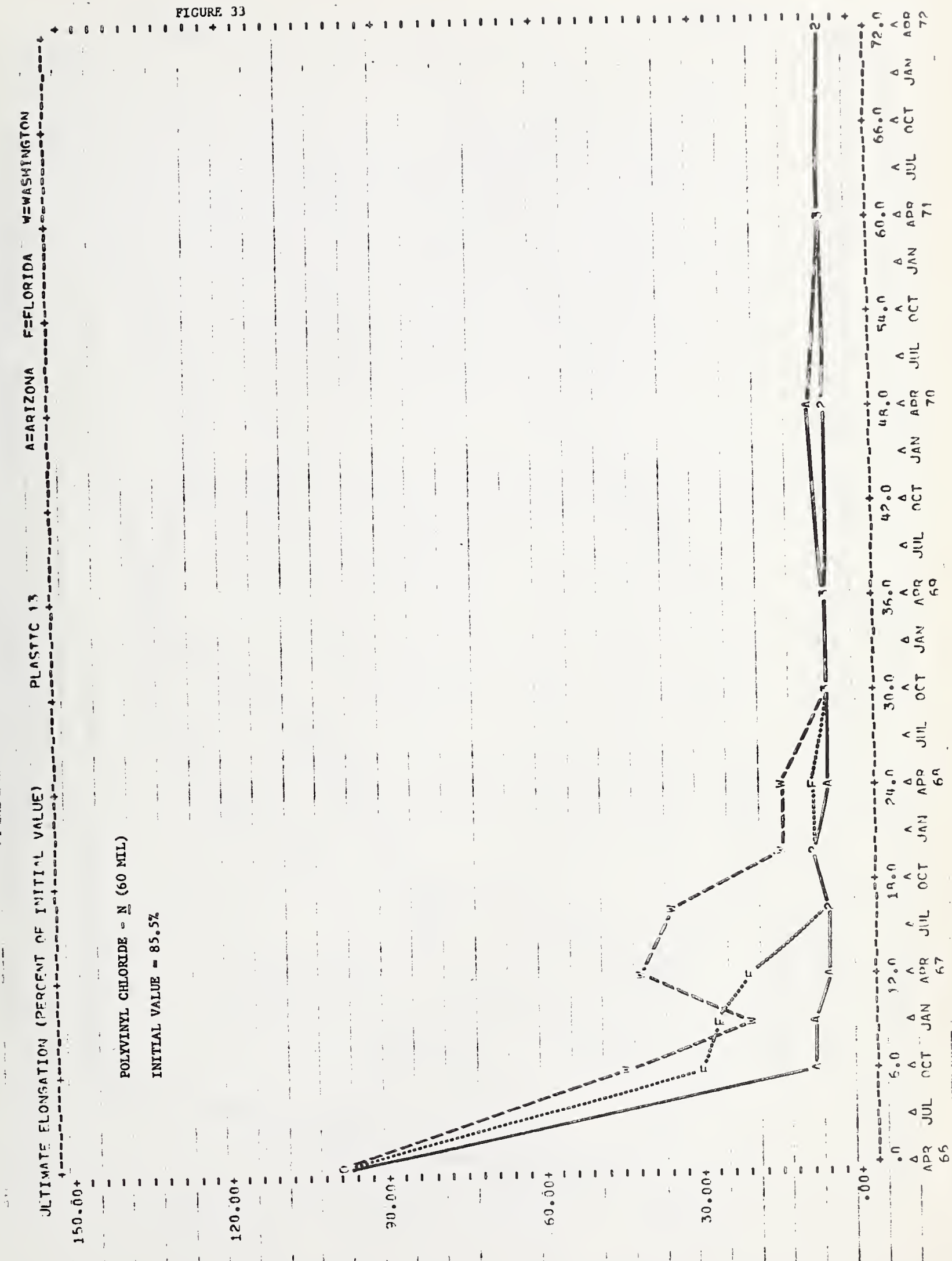


FIGURE 34

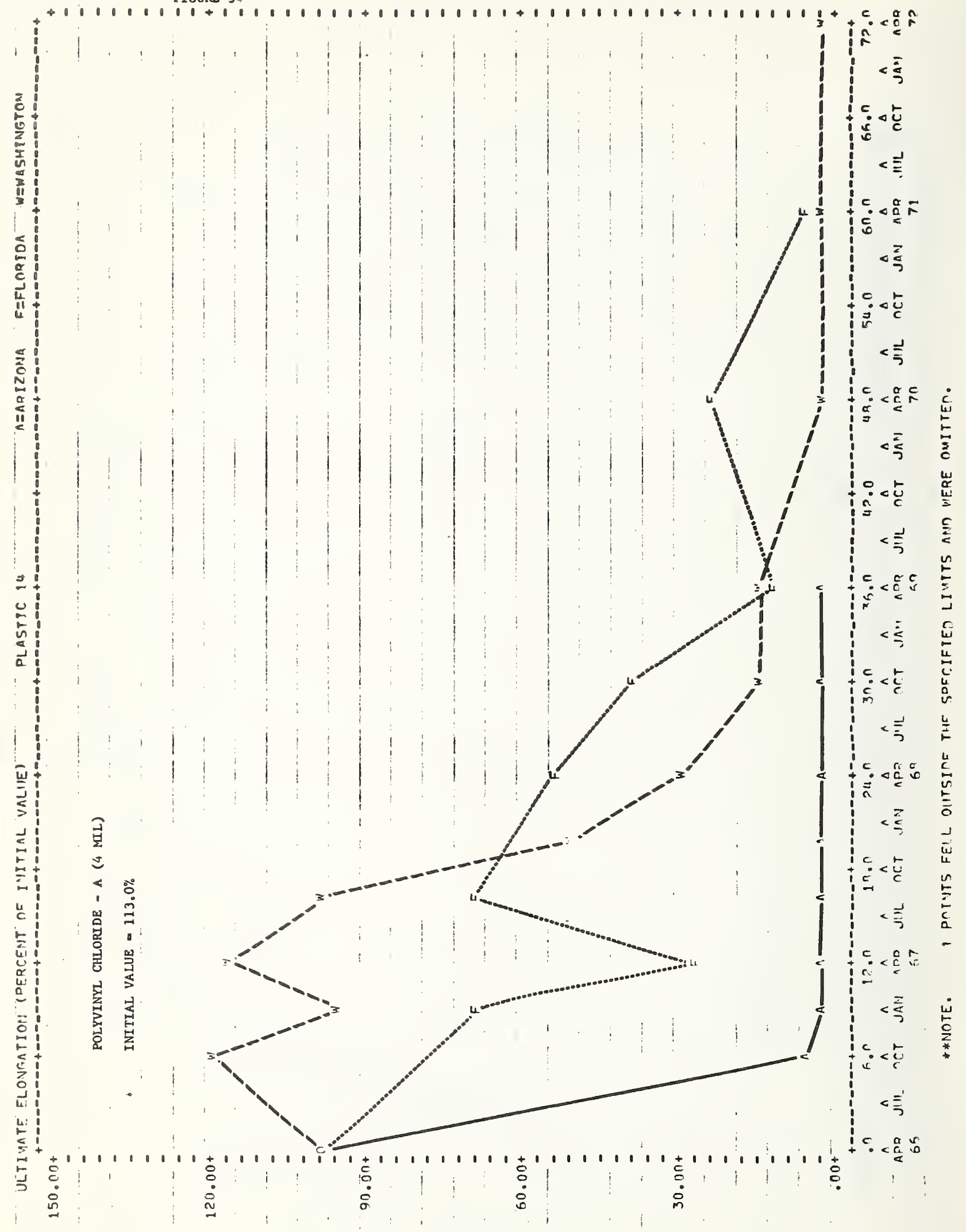


FIGURE 35

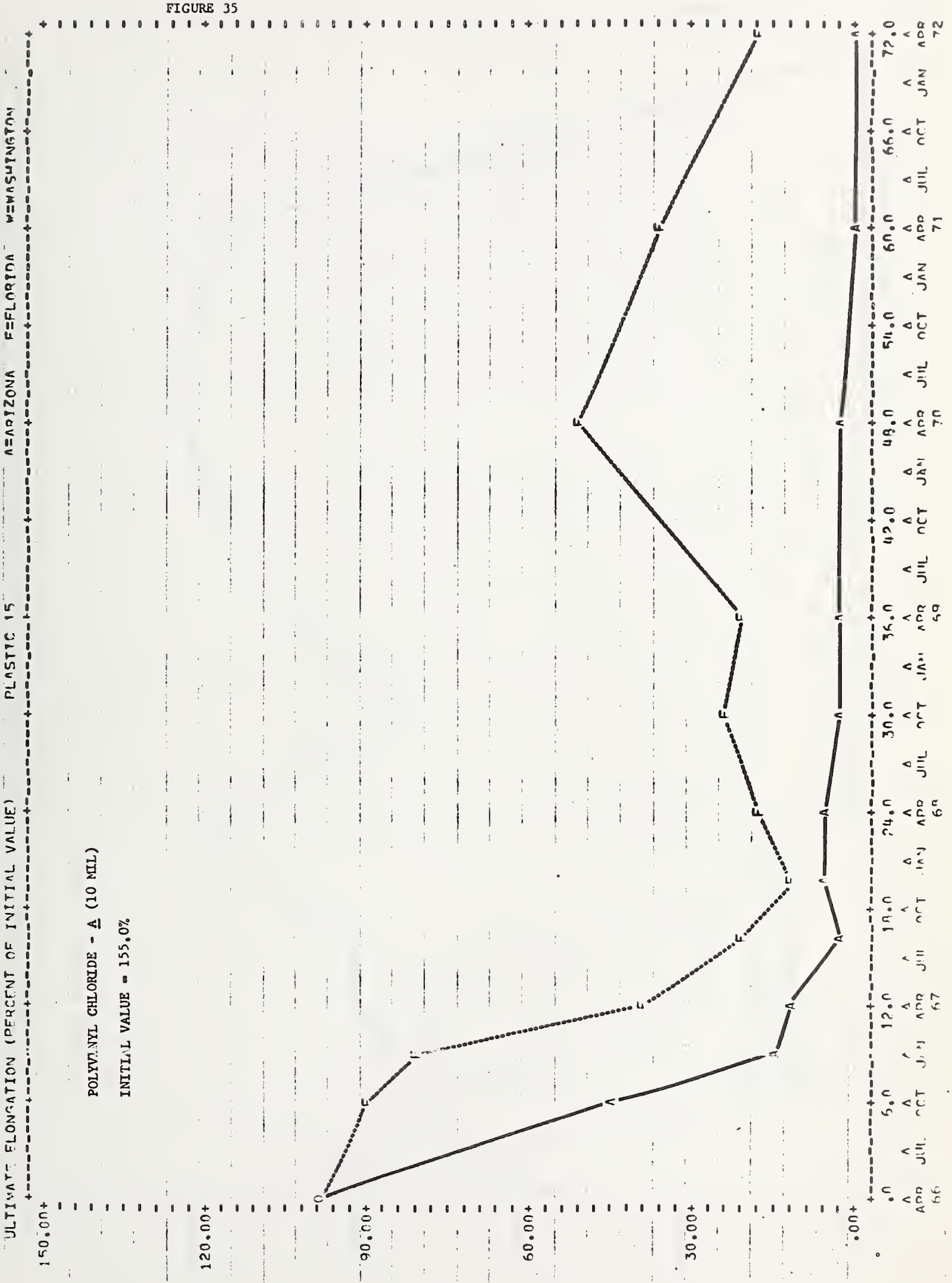


FIGURE 36

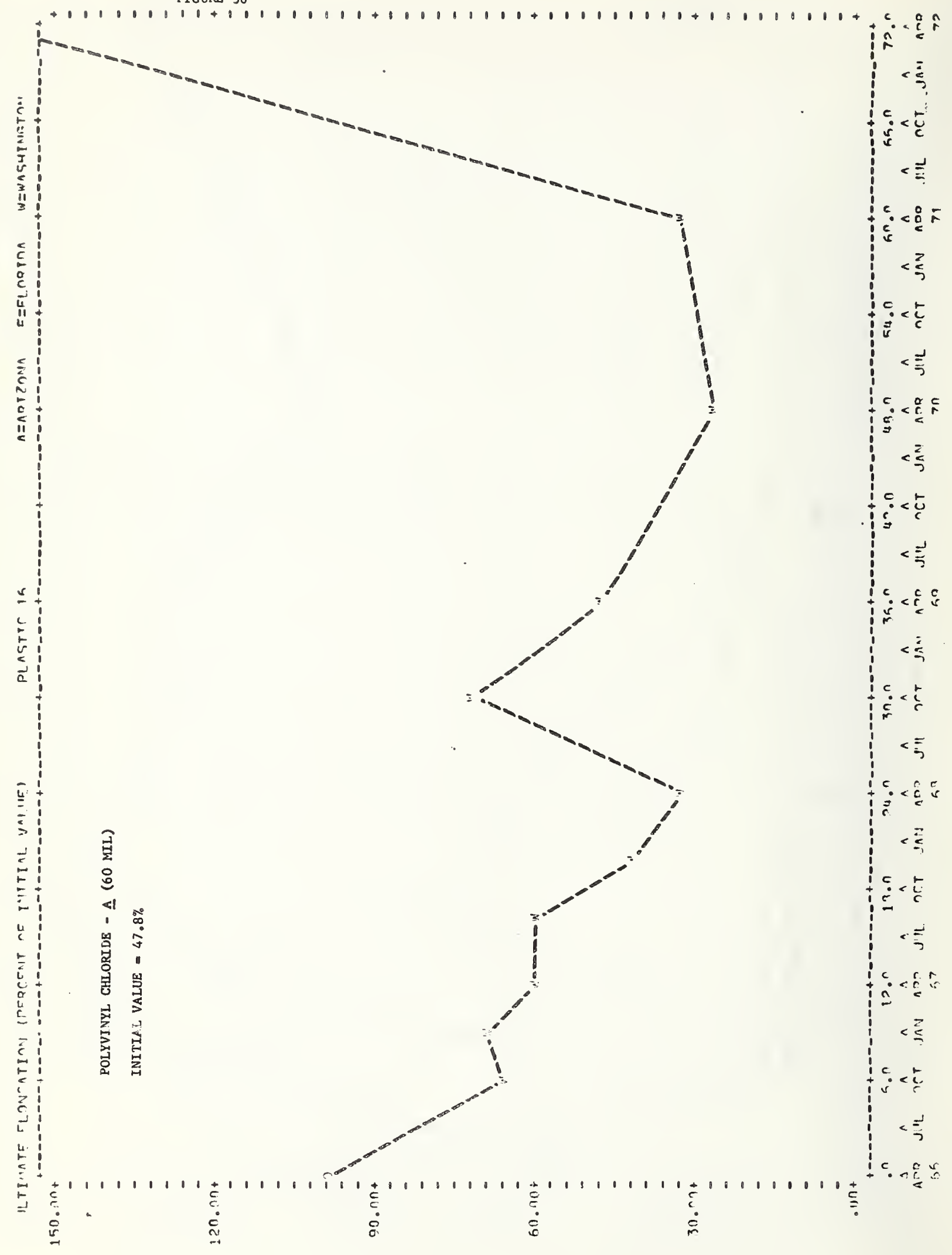


PIGURE 37

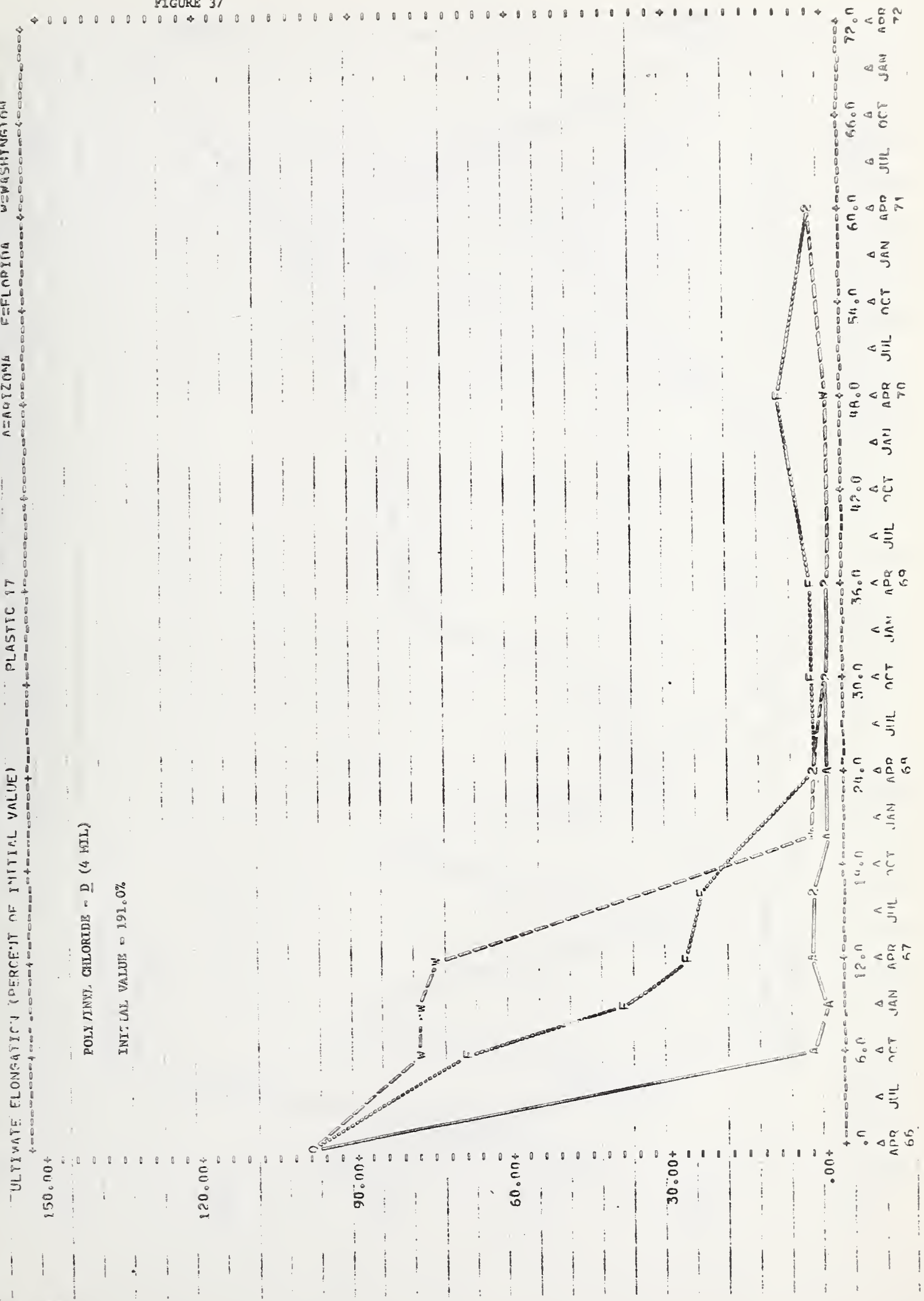


FTGURE 38

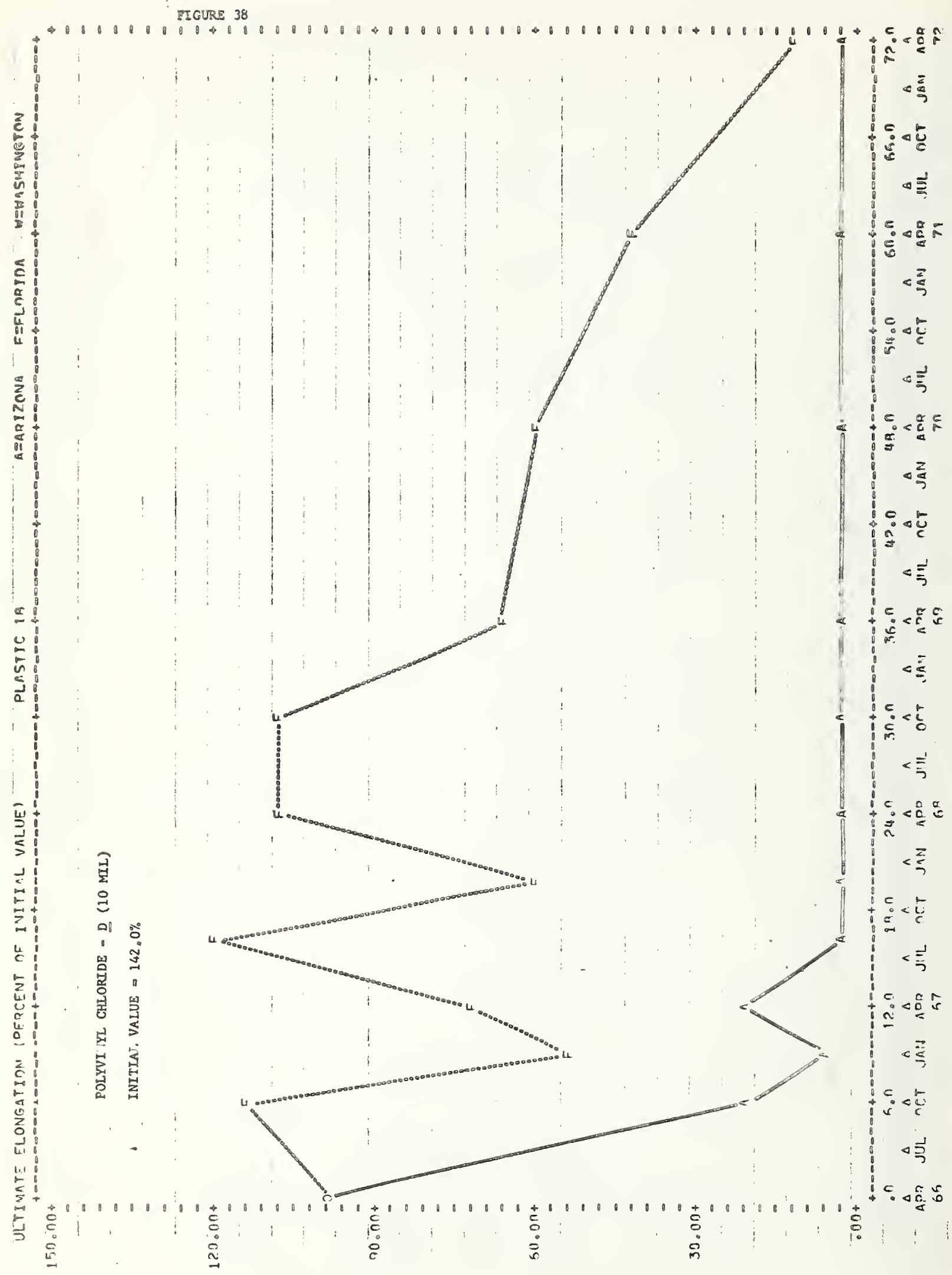


RIGURE 39

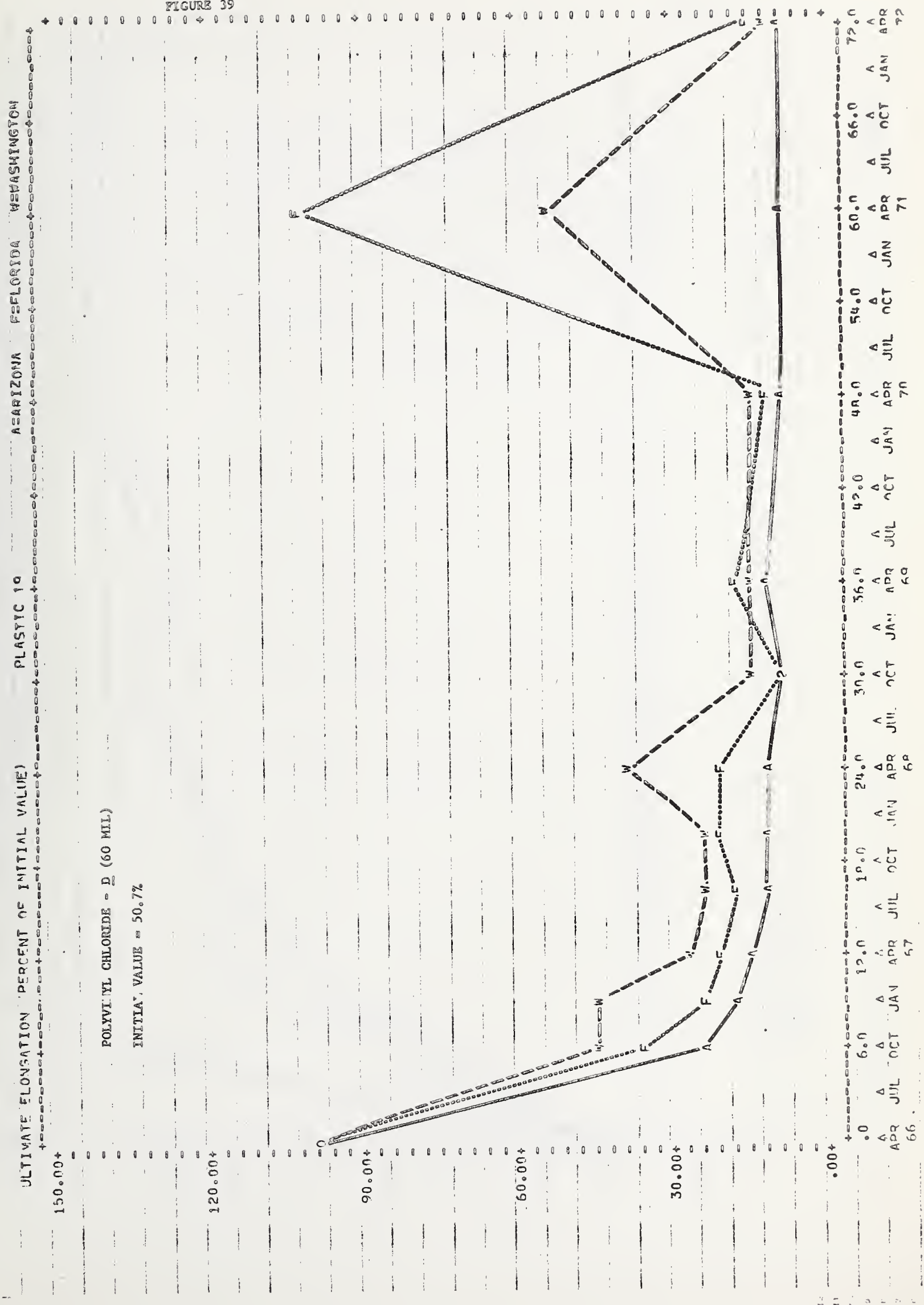


FIGURE 40

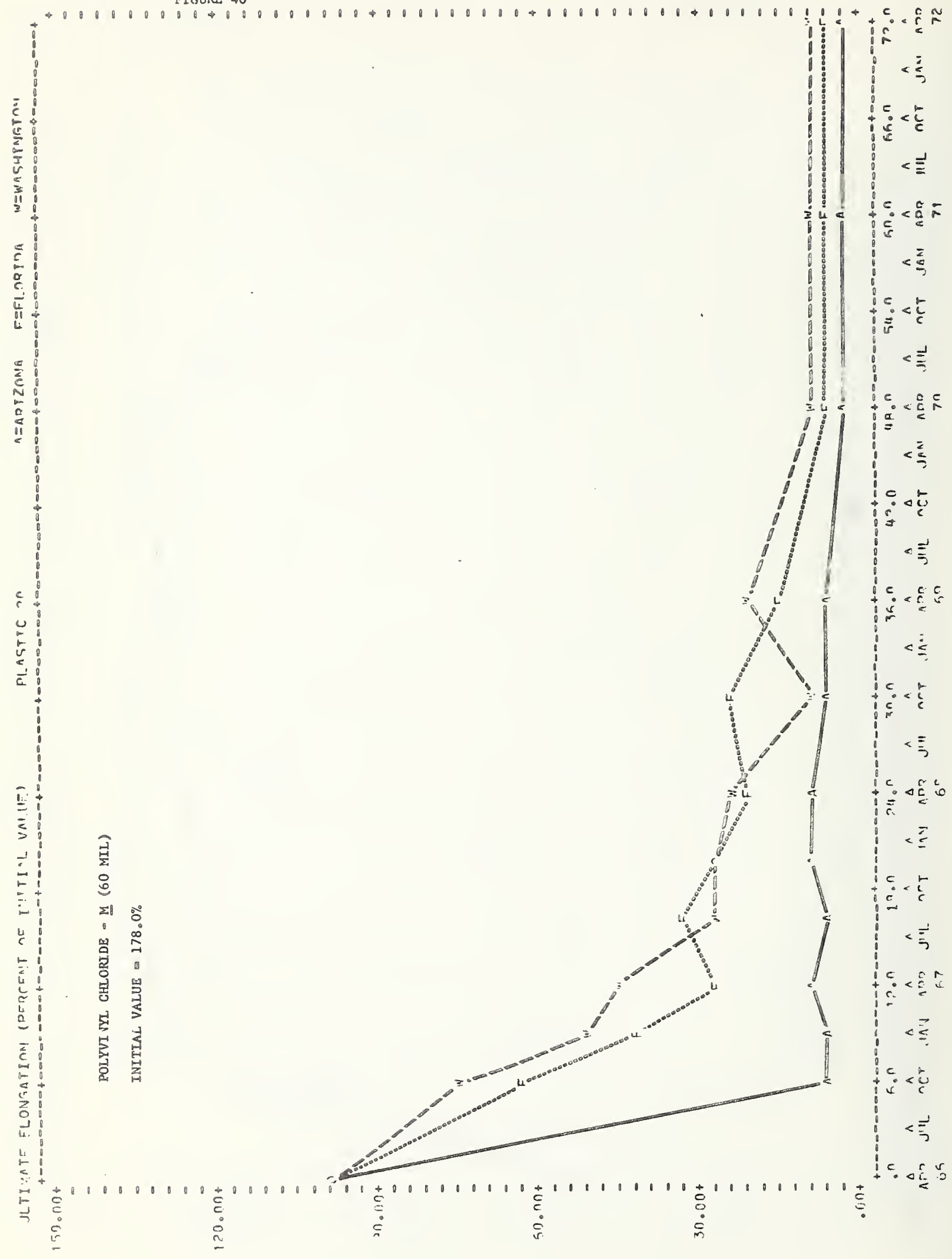


3IGURE $4 I$

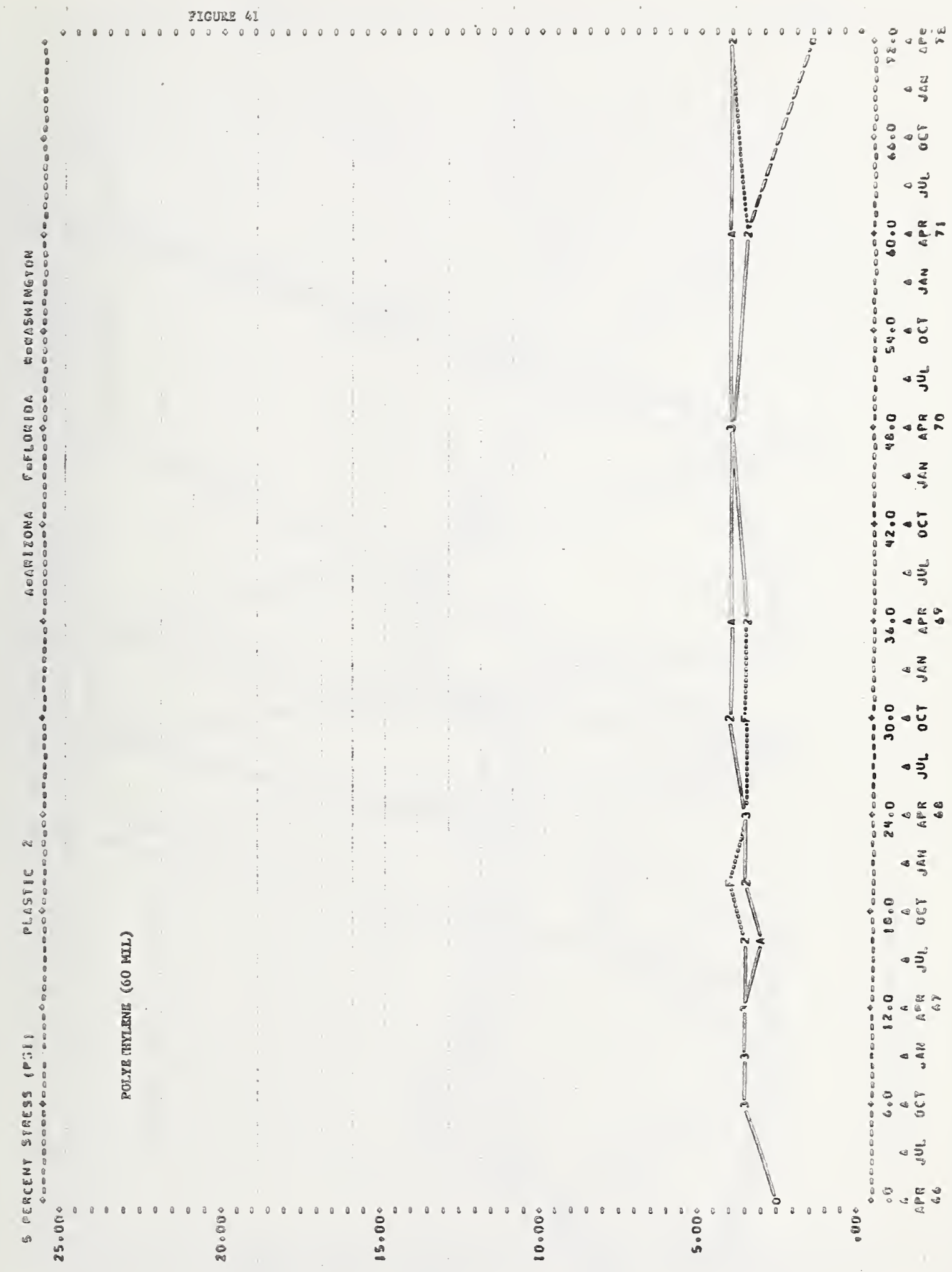


RIGURE 42

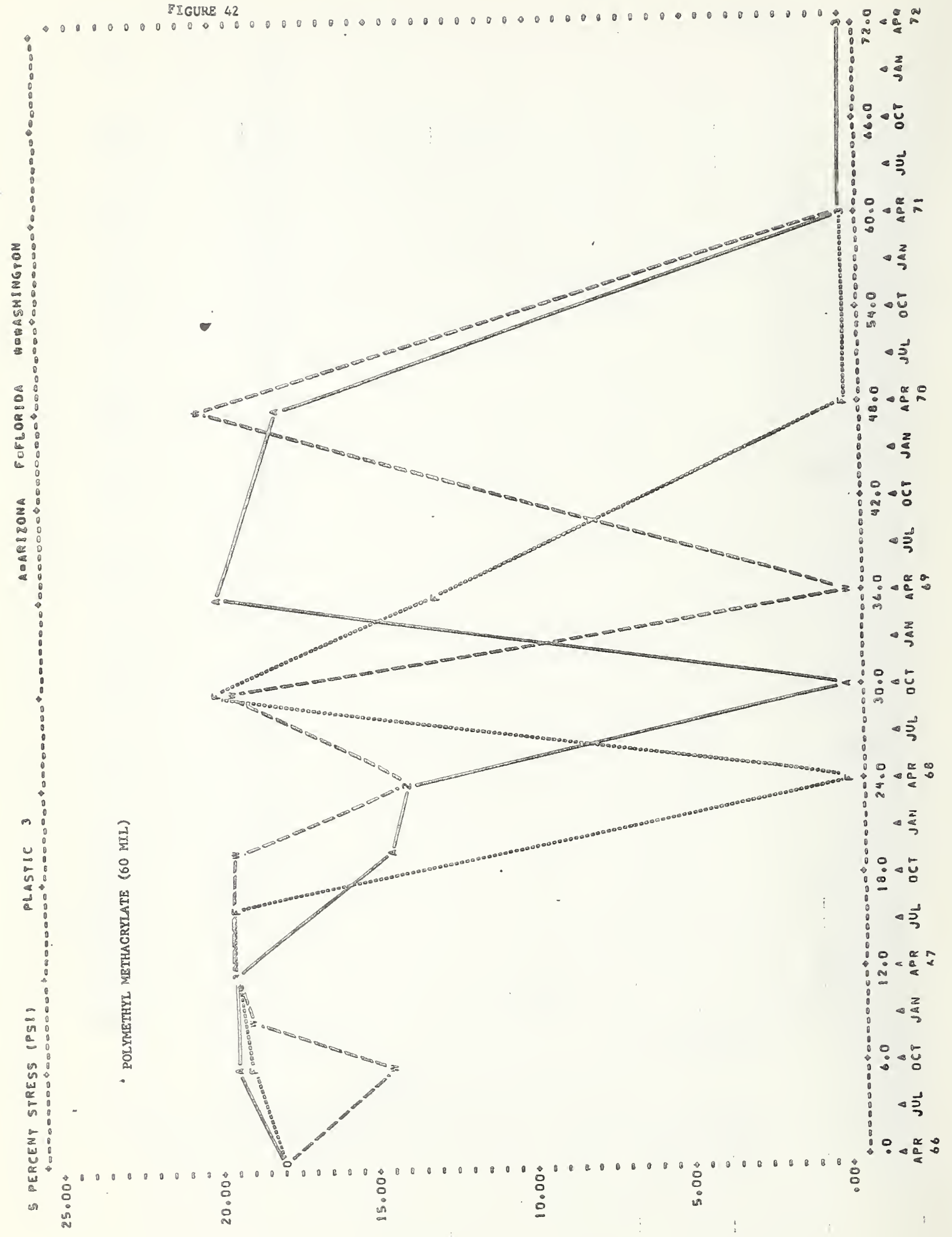


ELGURE 43

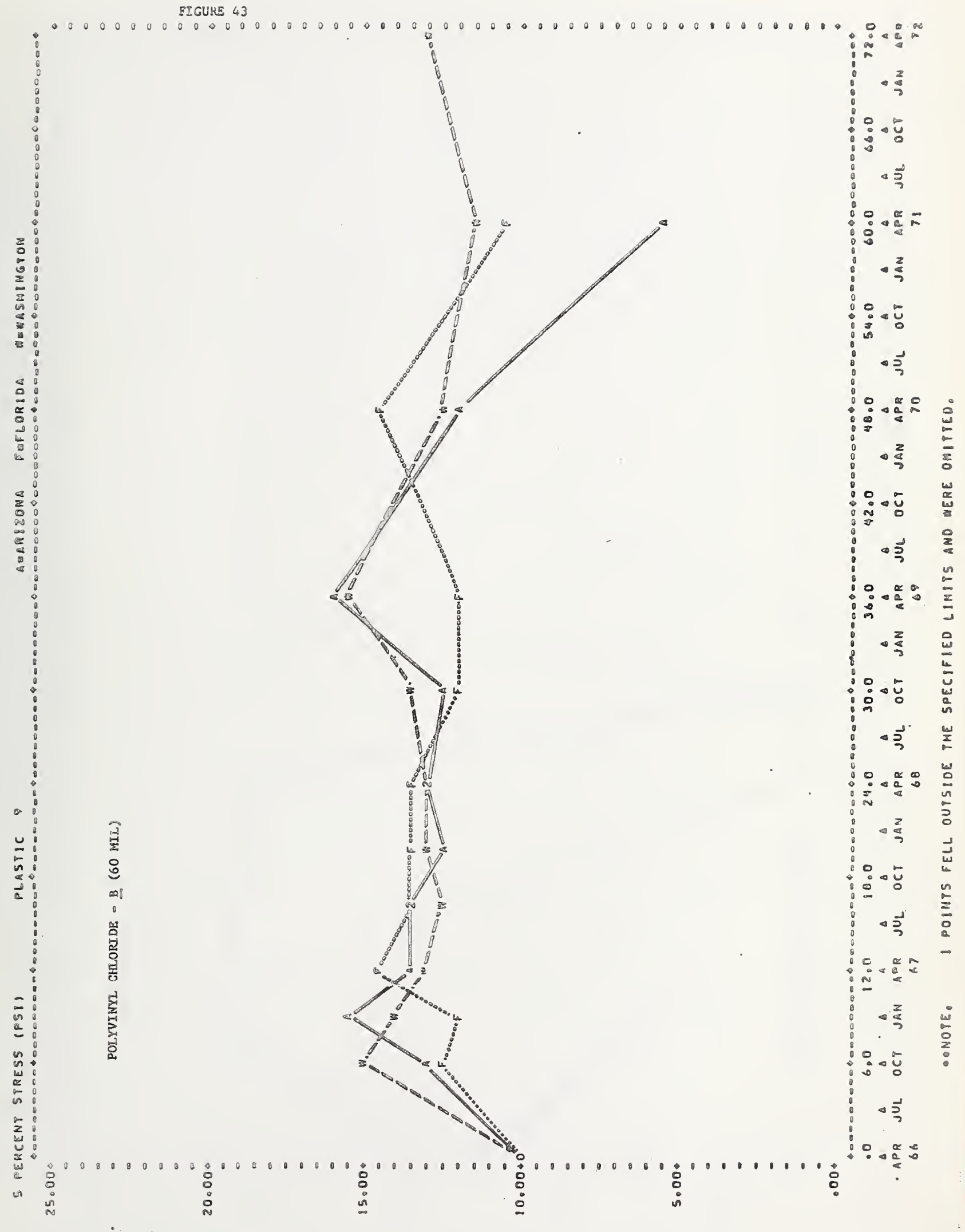




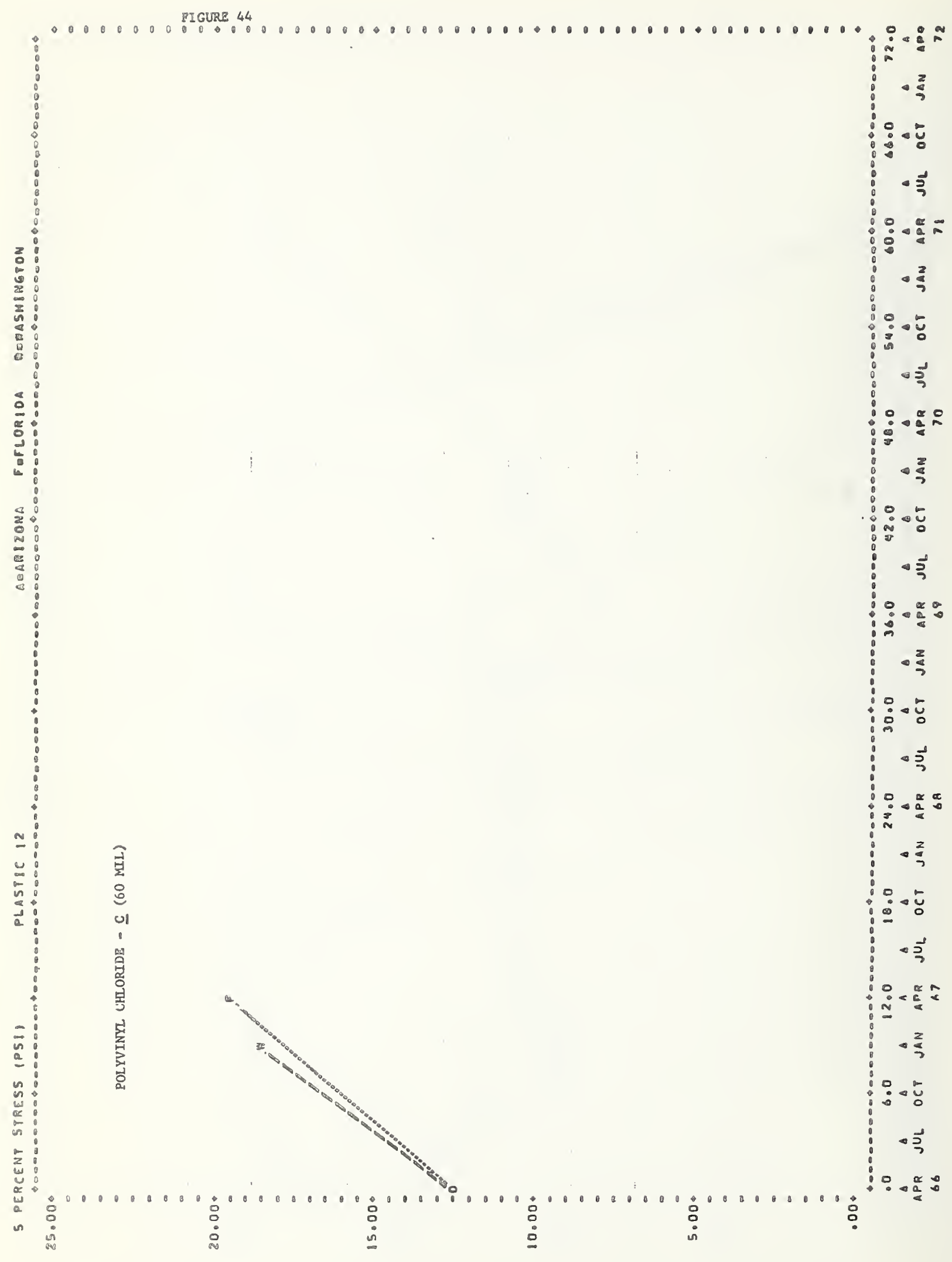


PIGURE 45

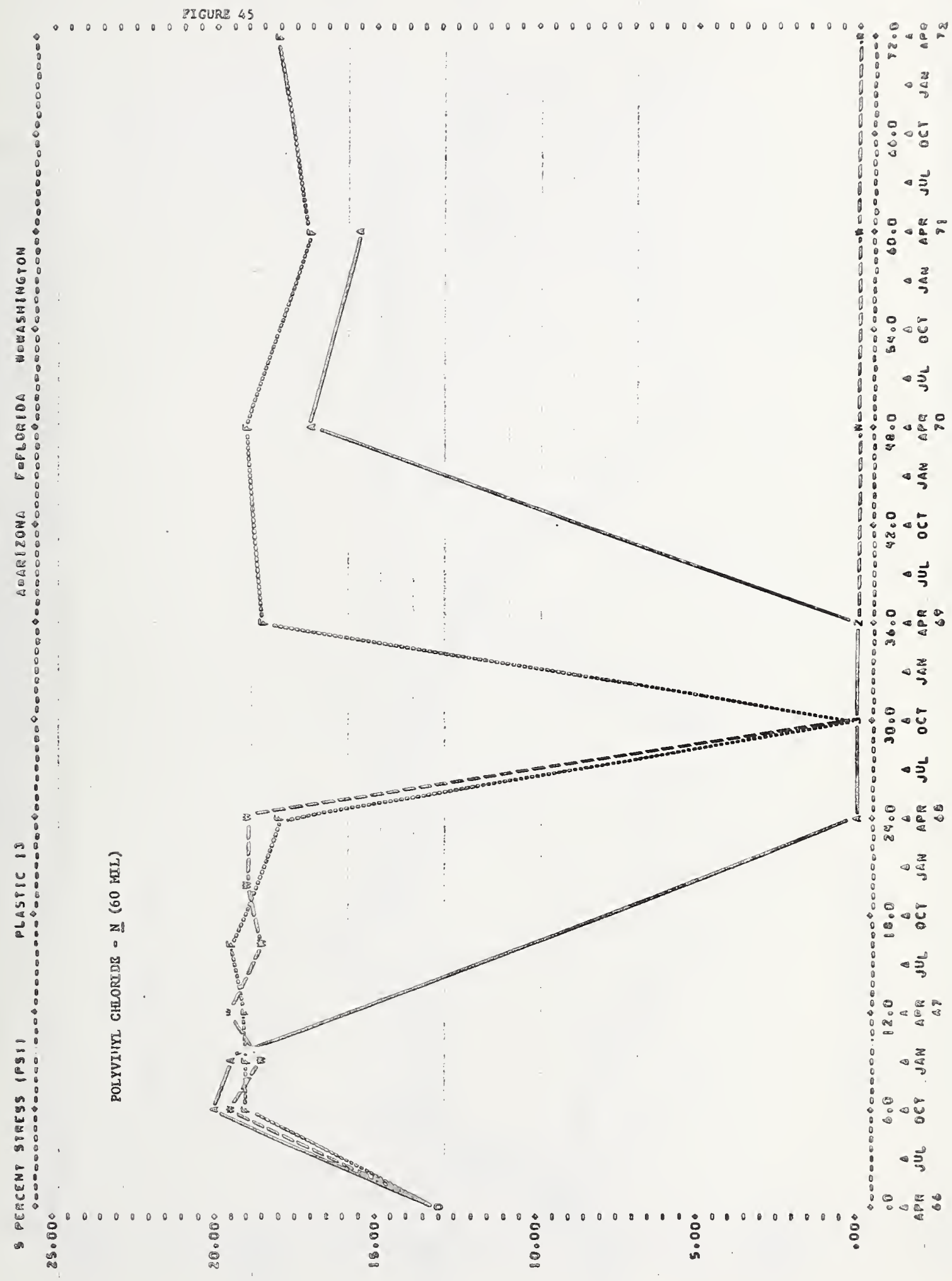


FIGURE 46

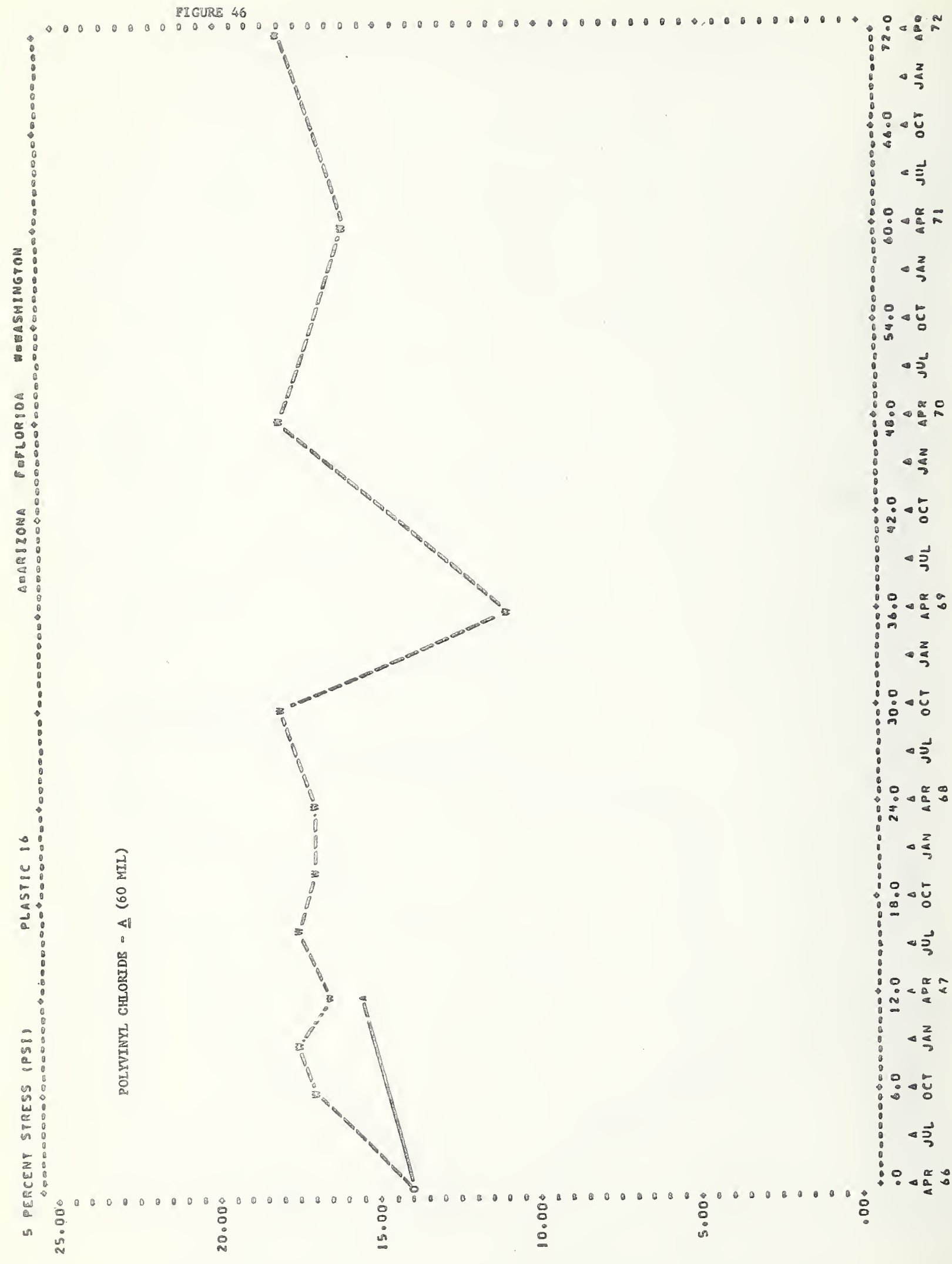




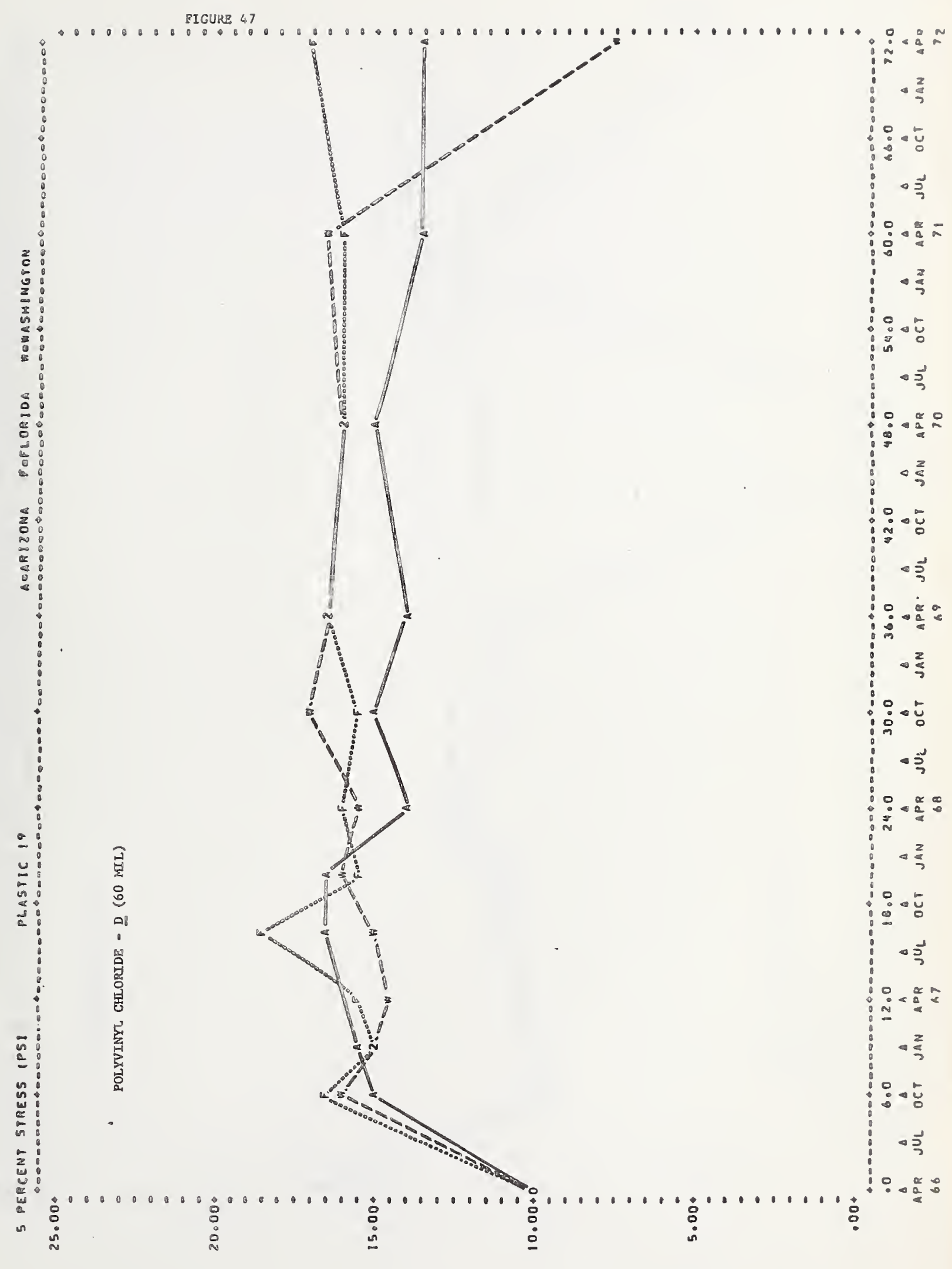




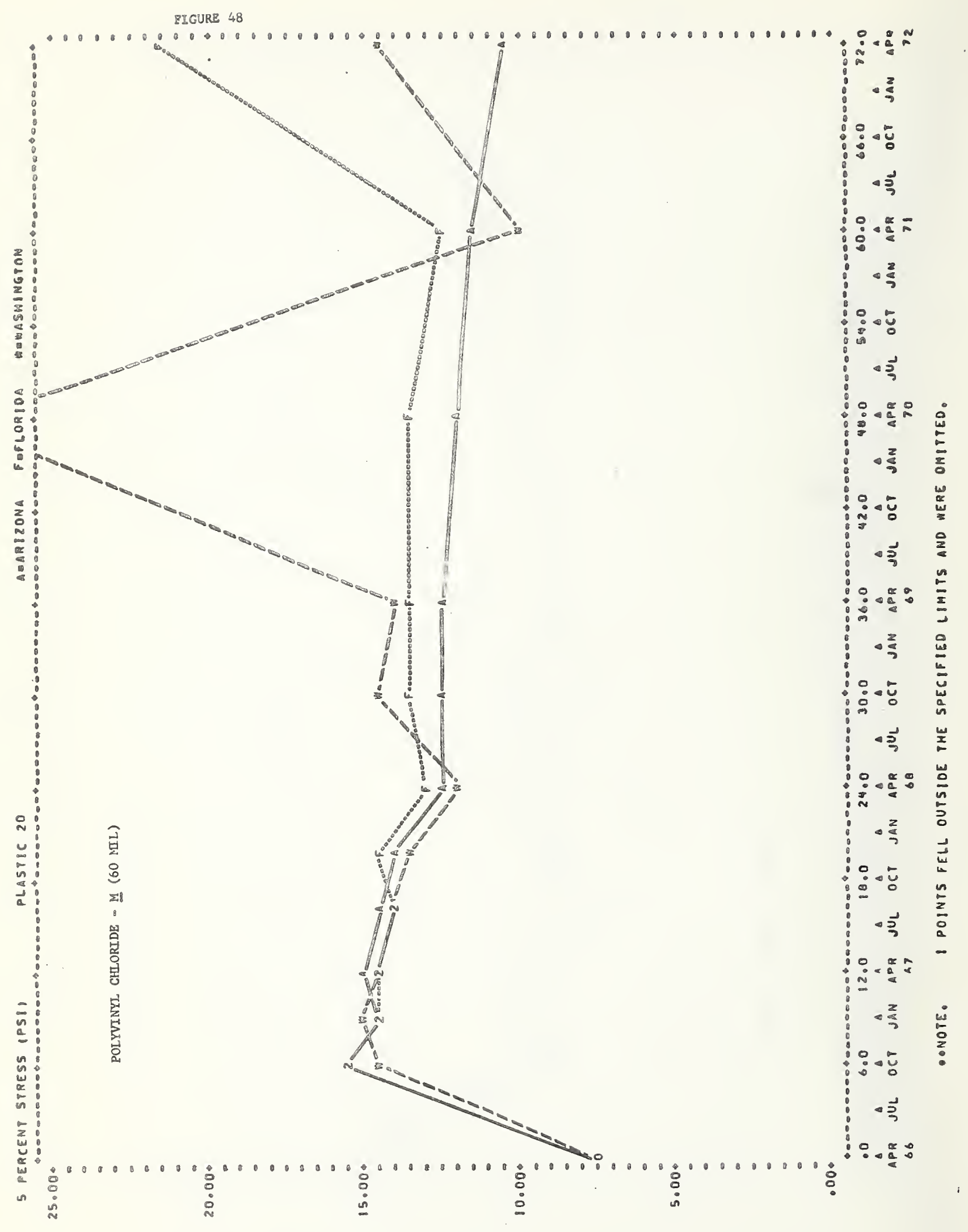


FIGURE $\div 9$

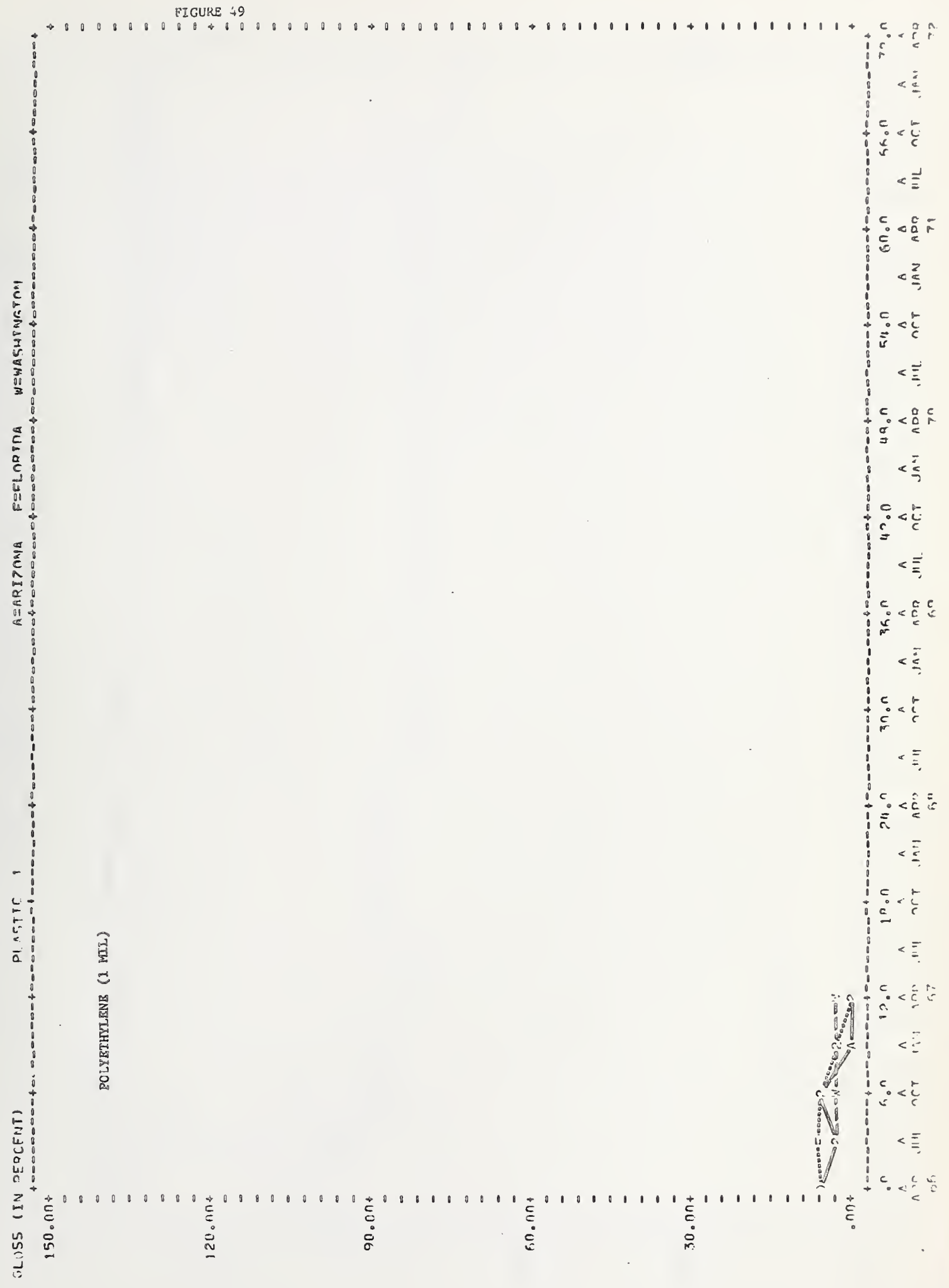


PIGURE SO

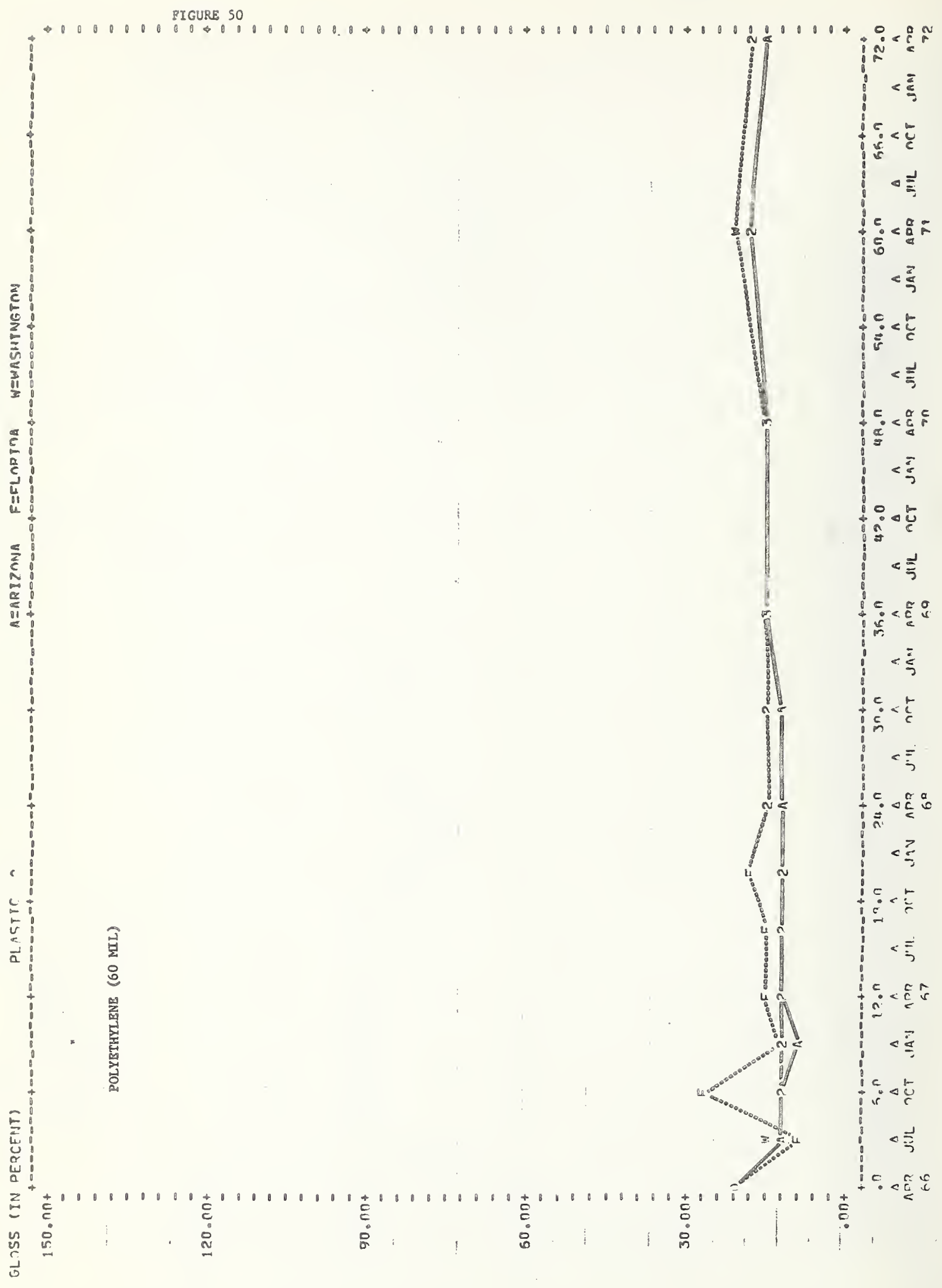




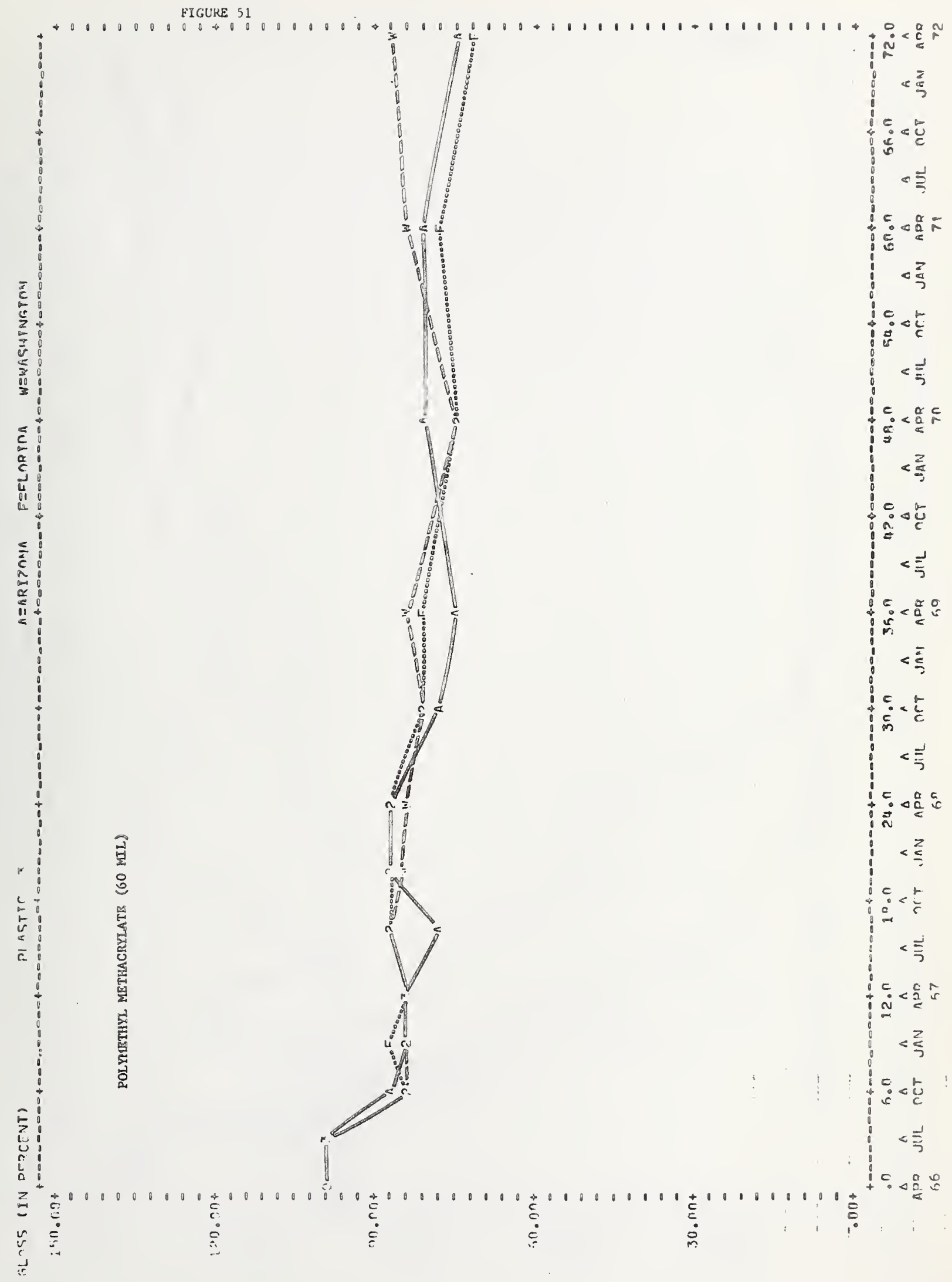


FIGURE 52

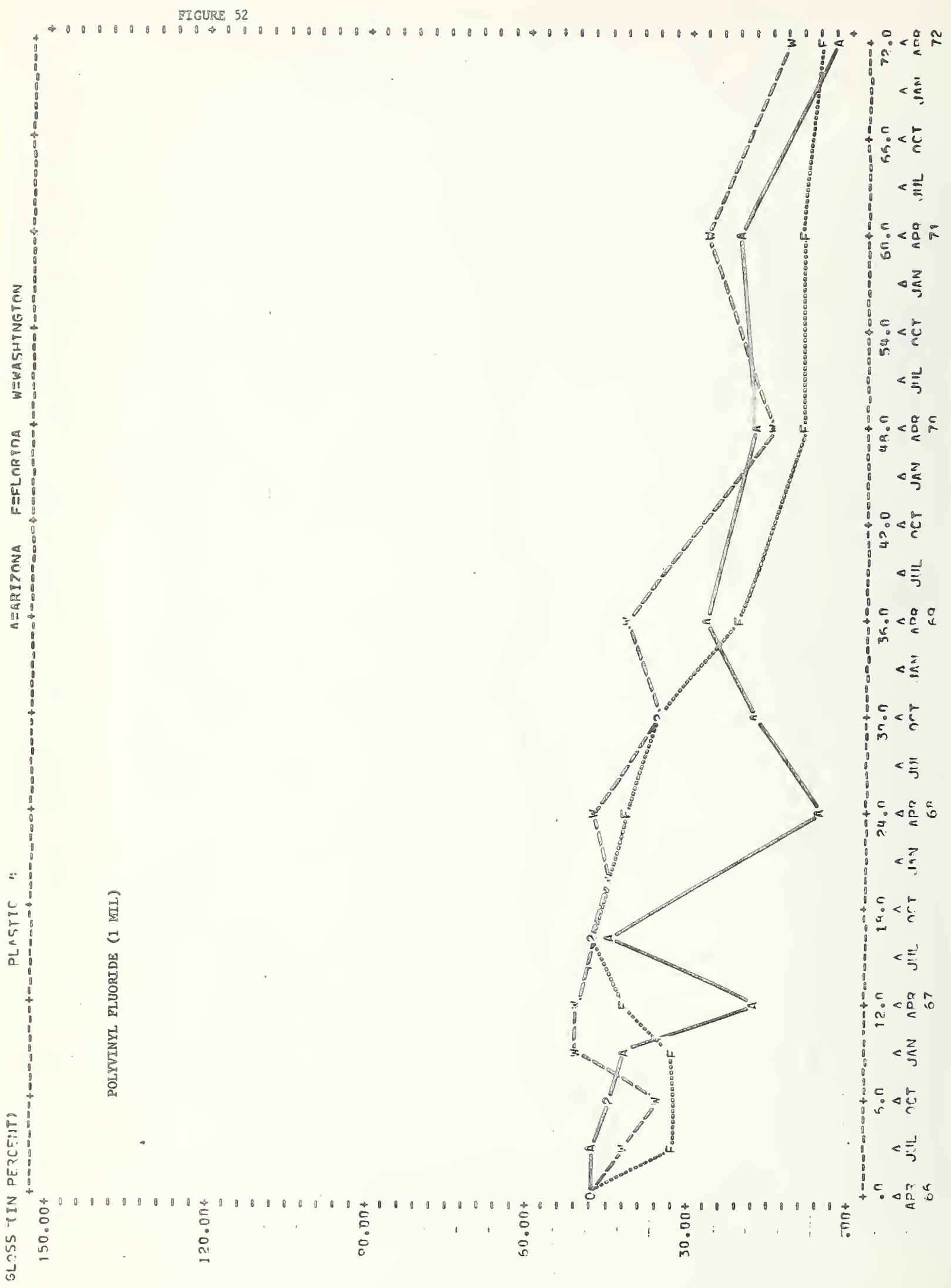




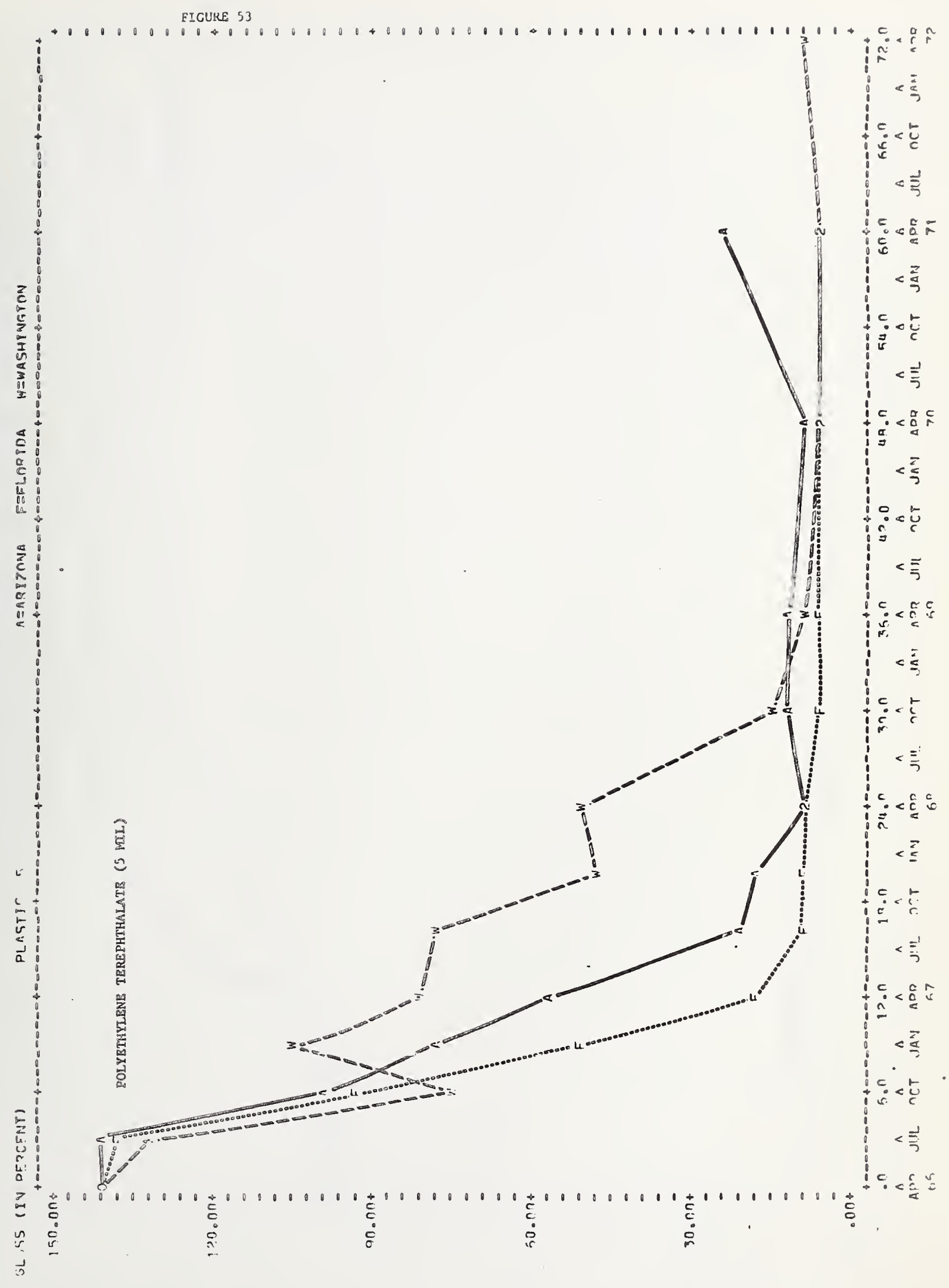


FIGURE 54

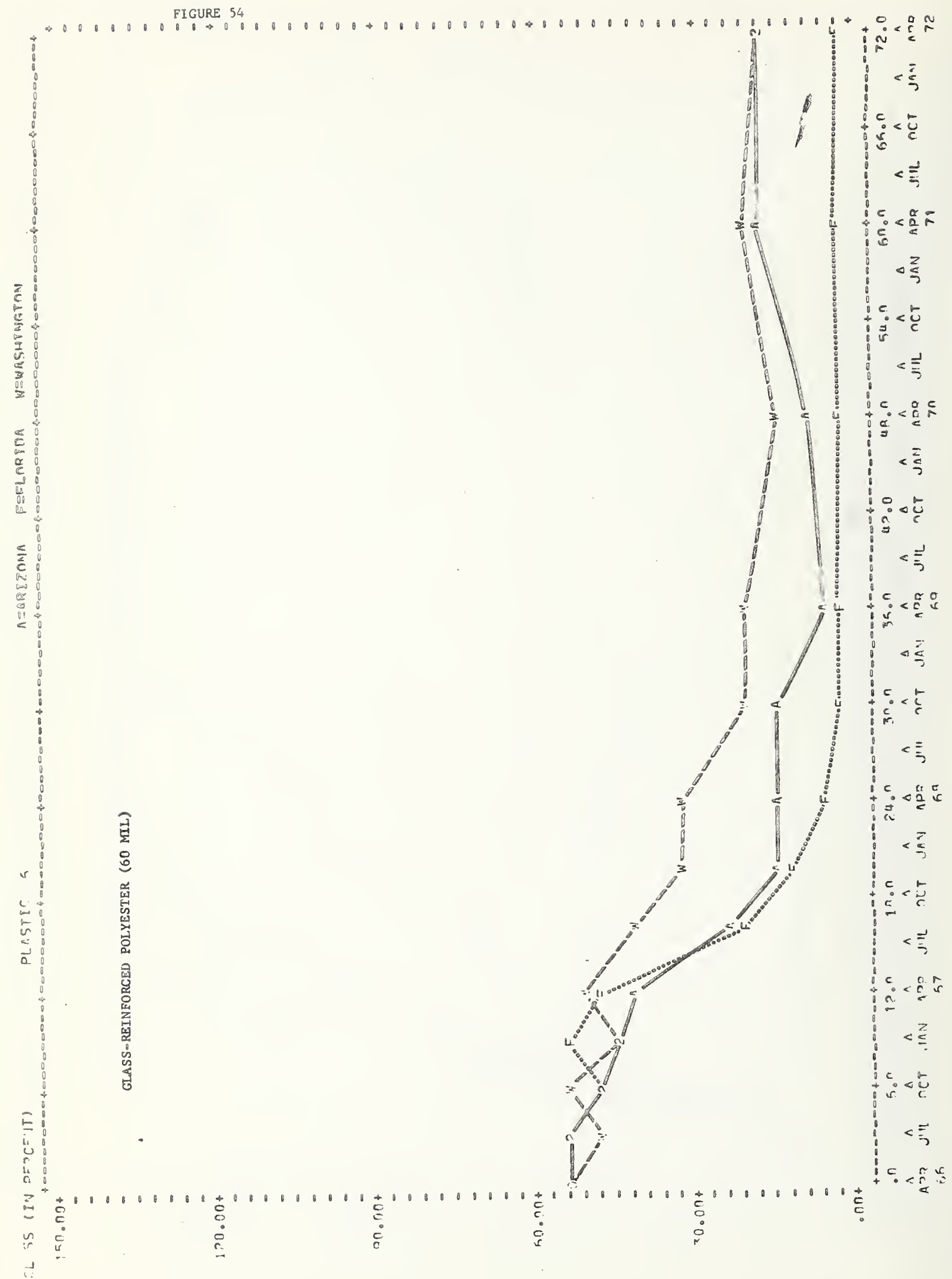




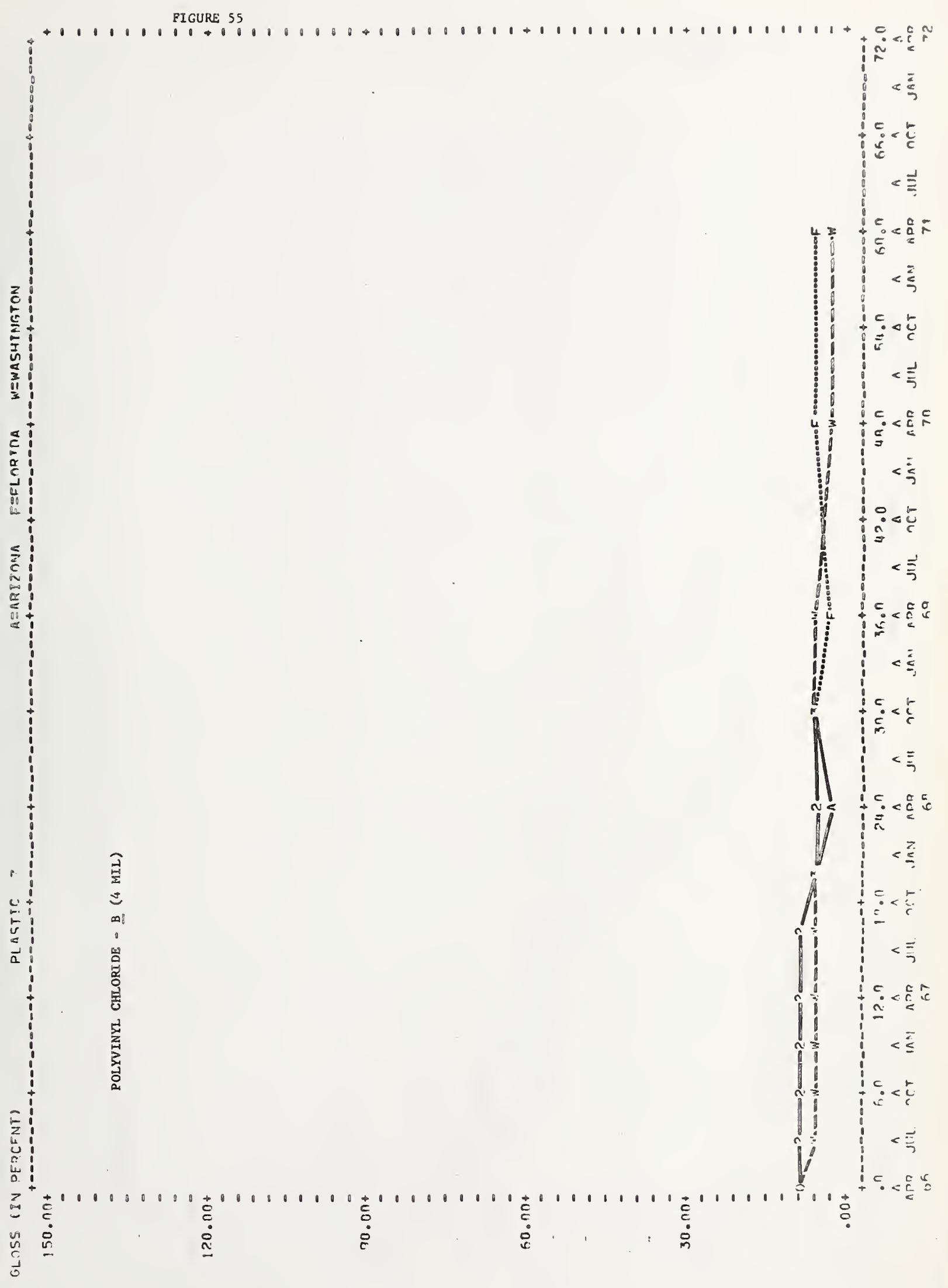




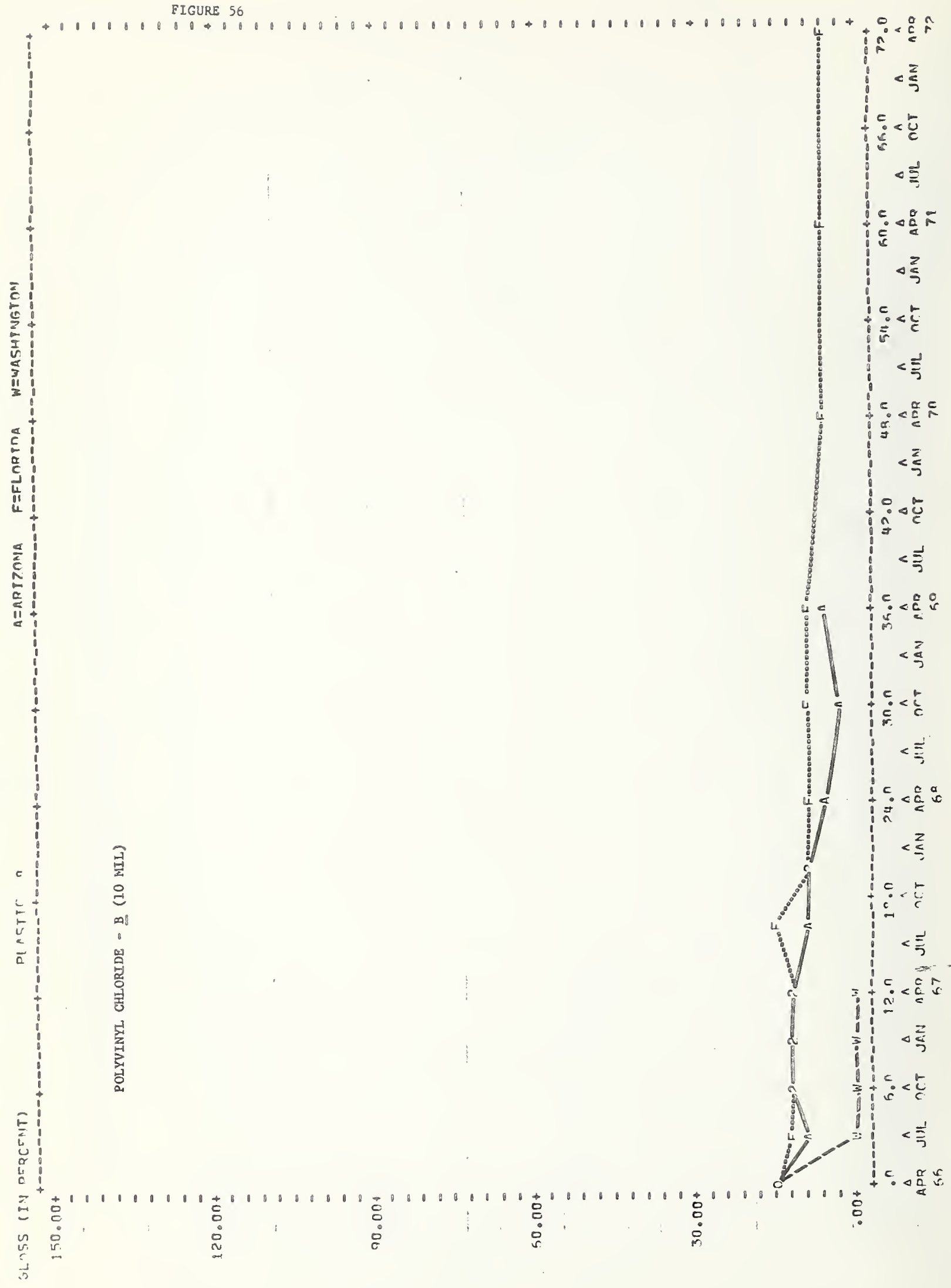


FIGURE 57

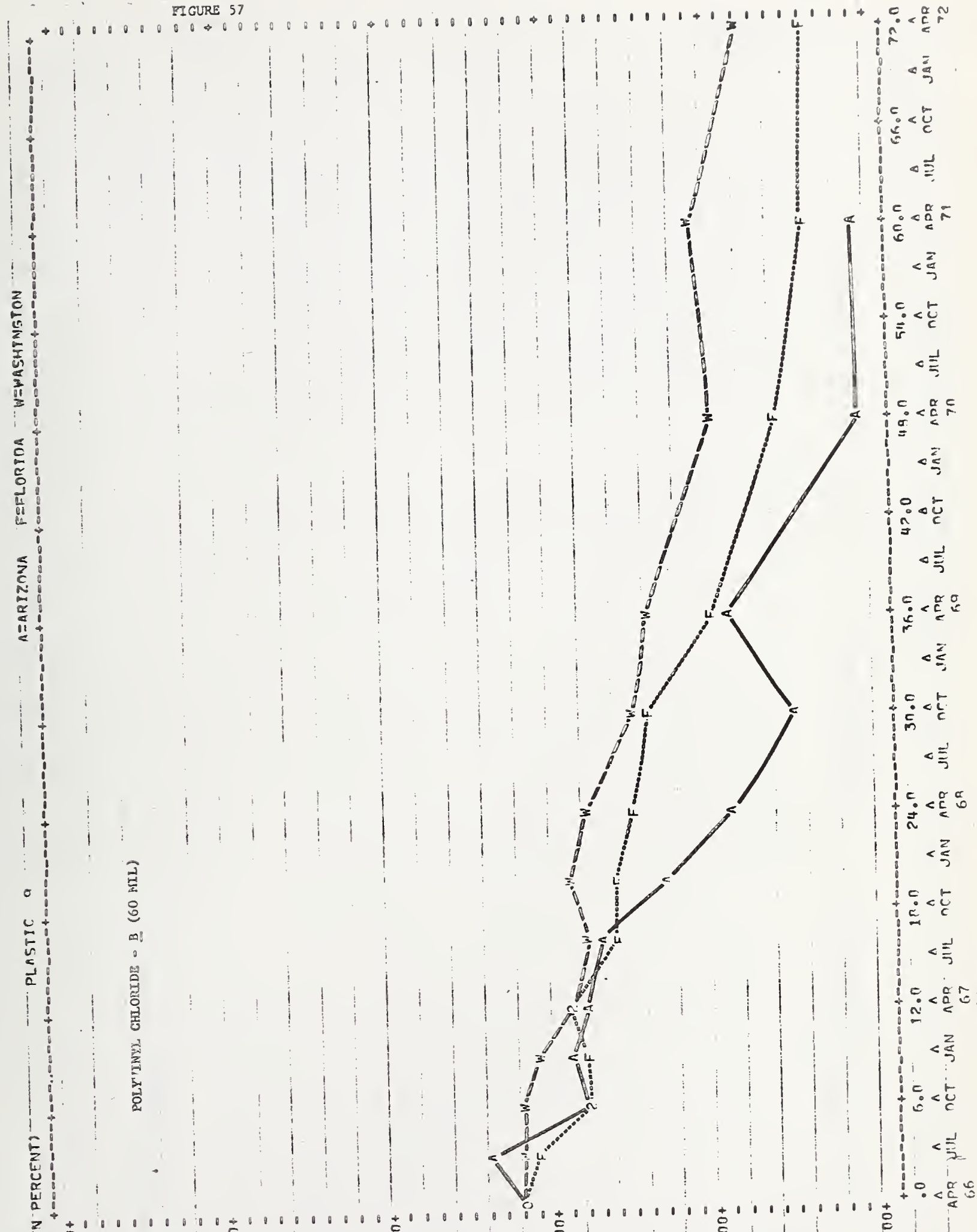

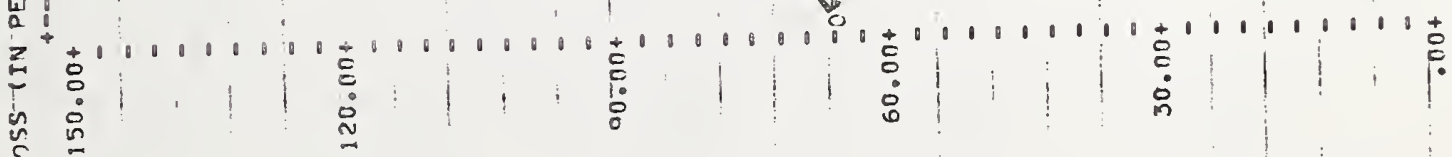


EIGURE SB

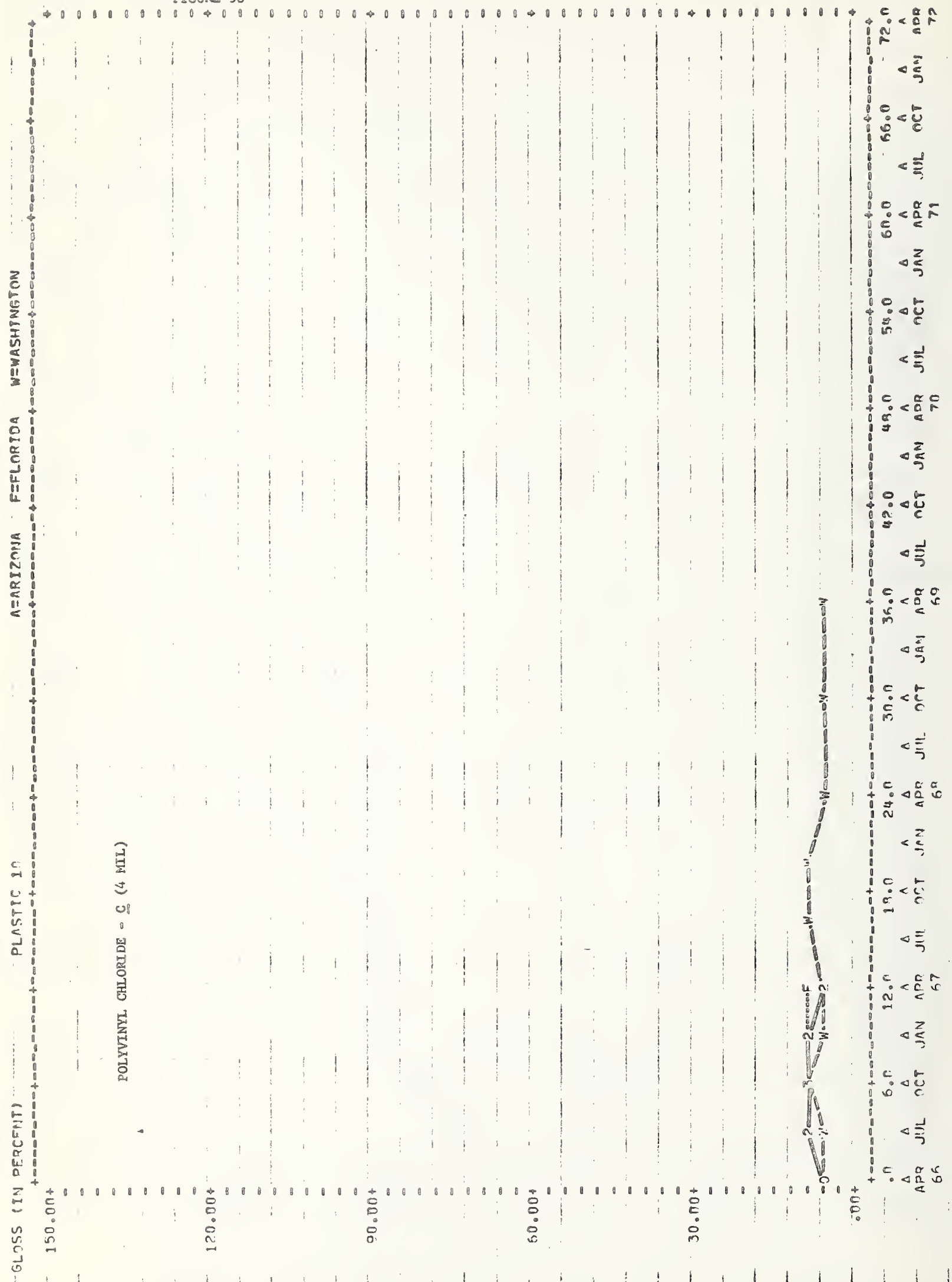


MGURE 59

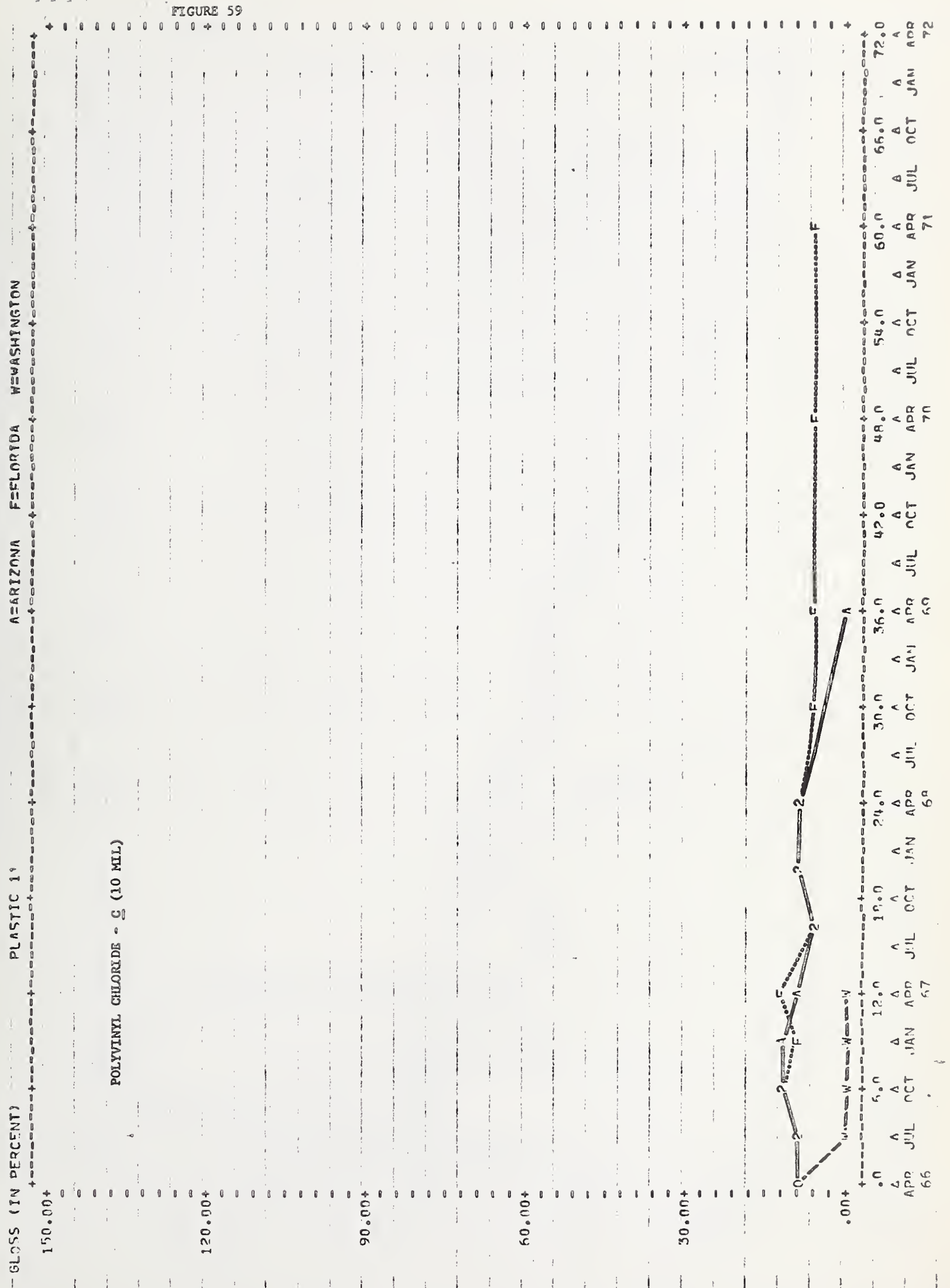


PIGURE 60

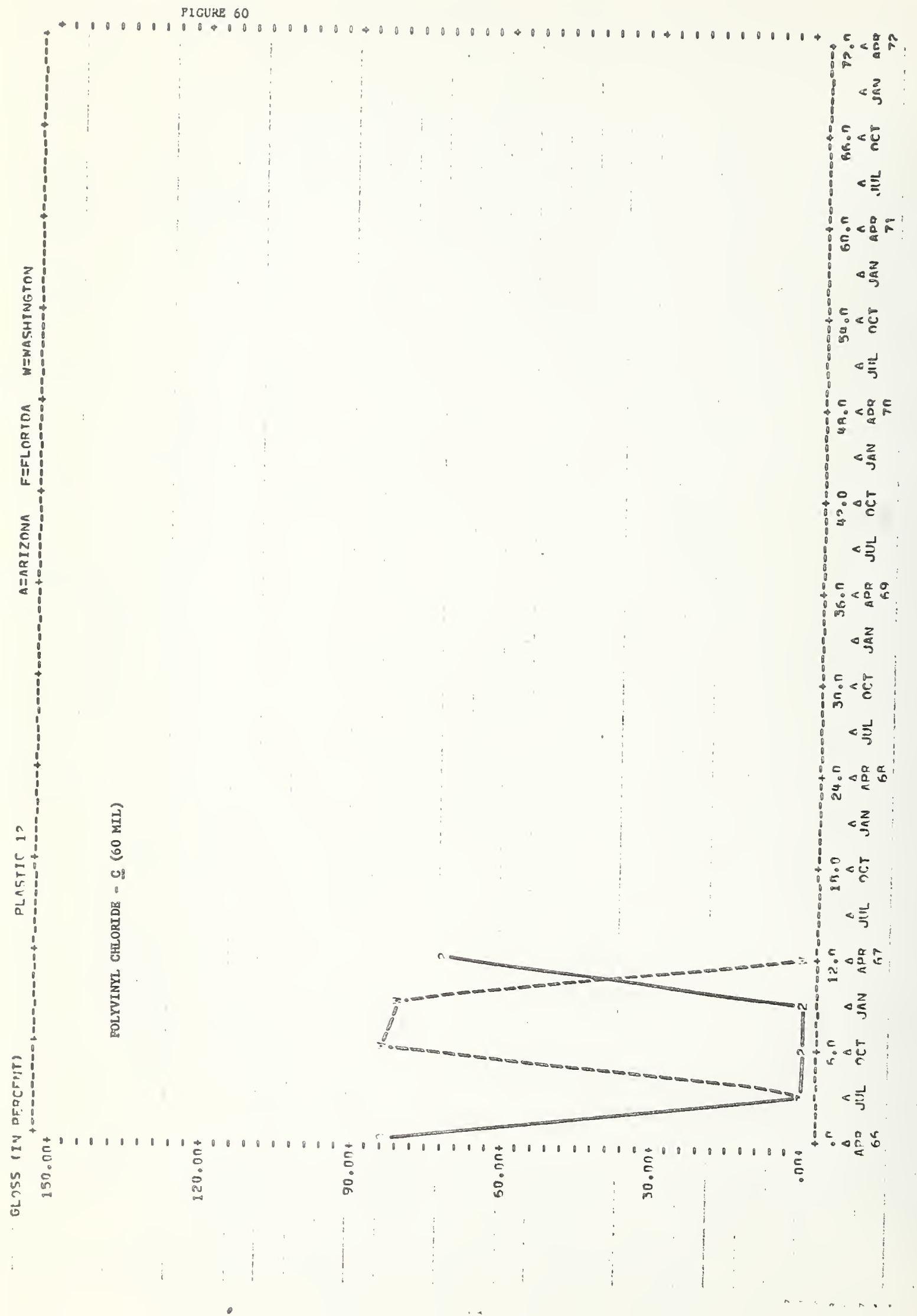


ICURE 61

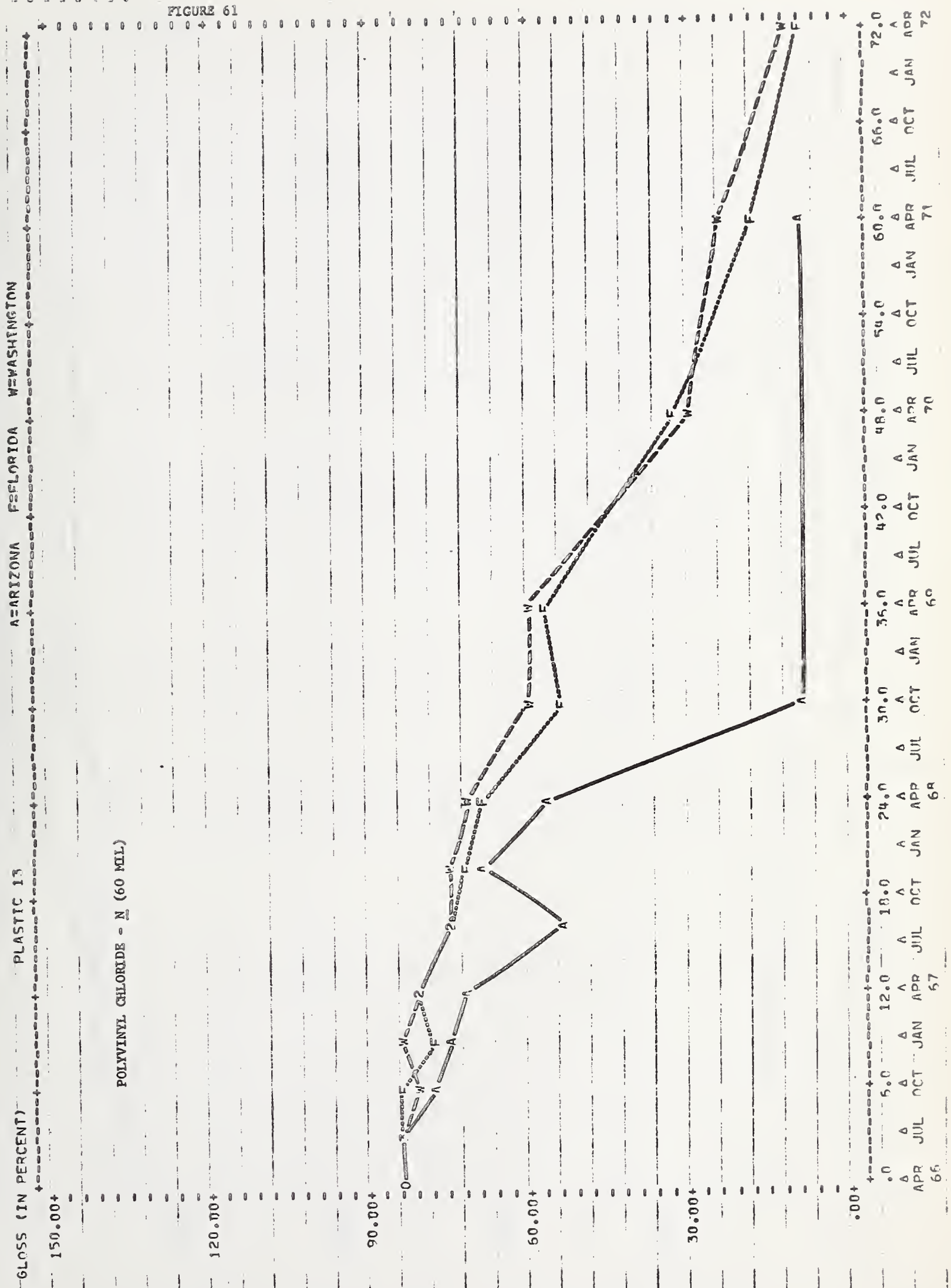


PI GURE 62

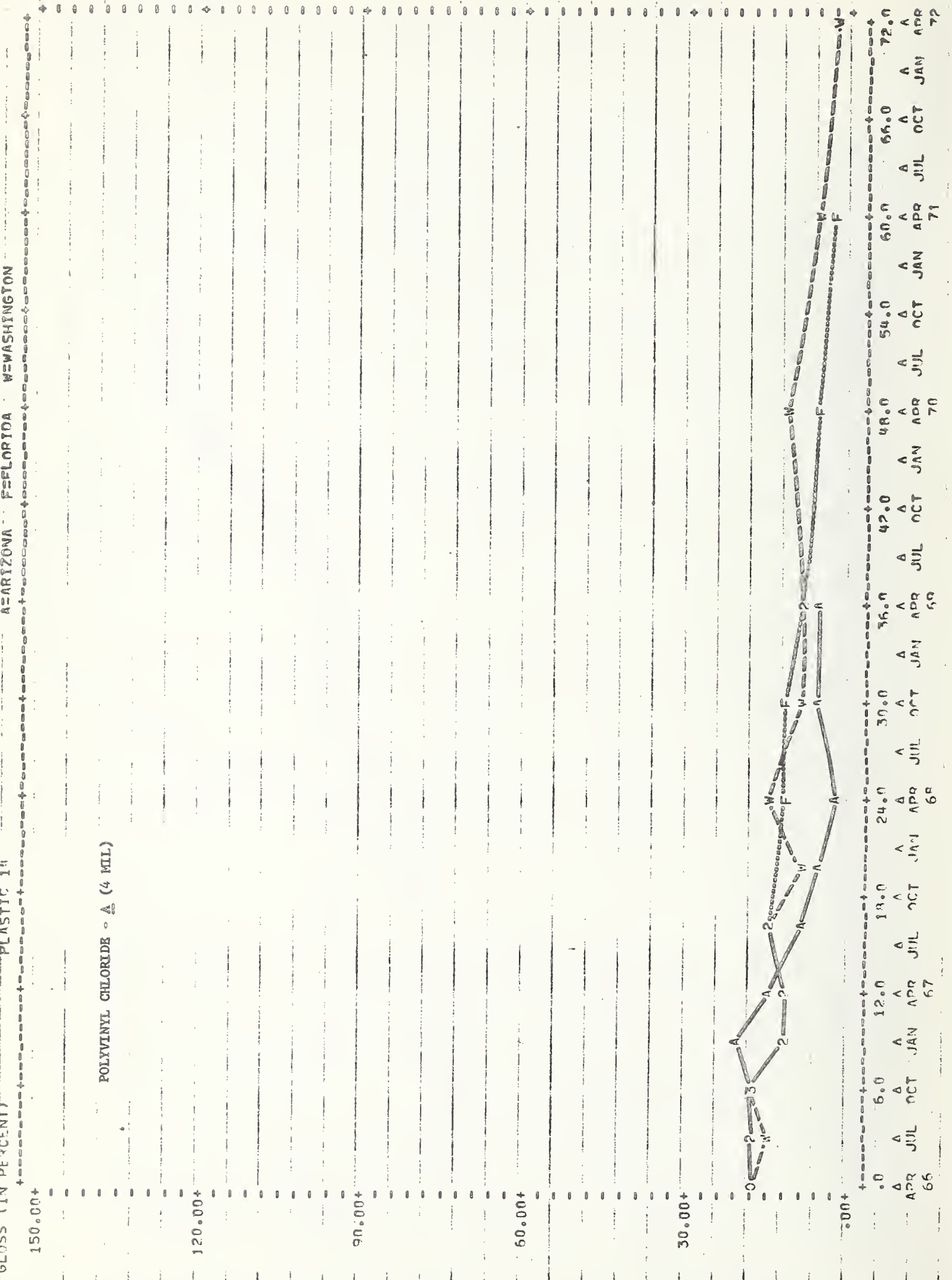


FIGURE 63

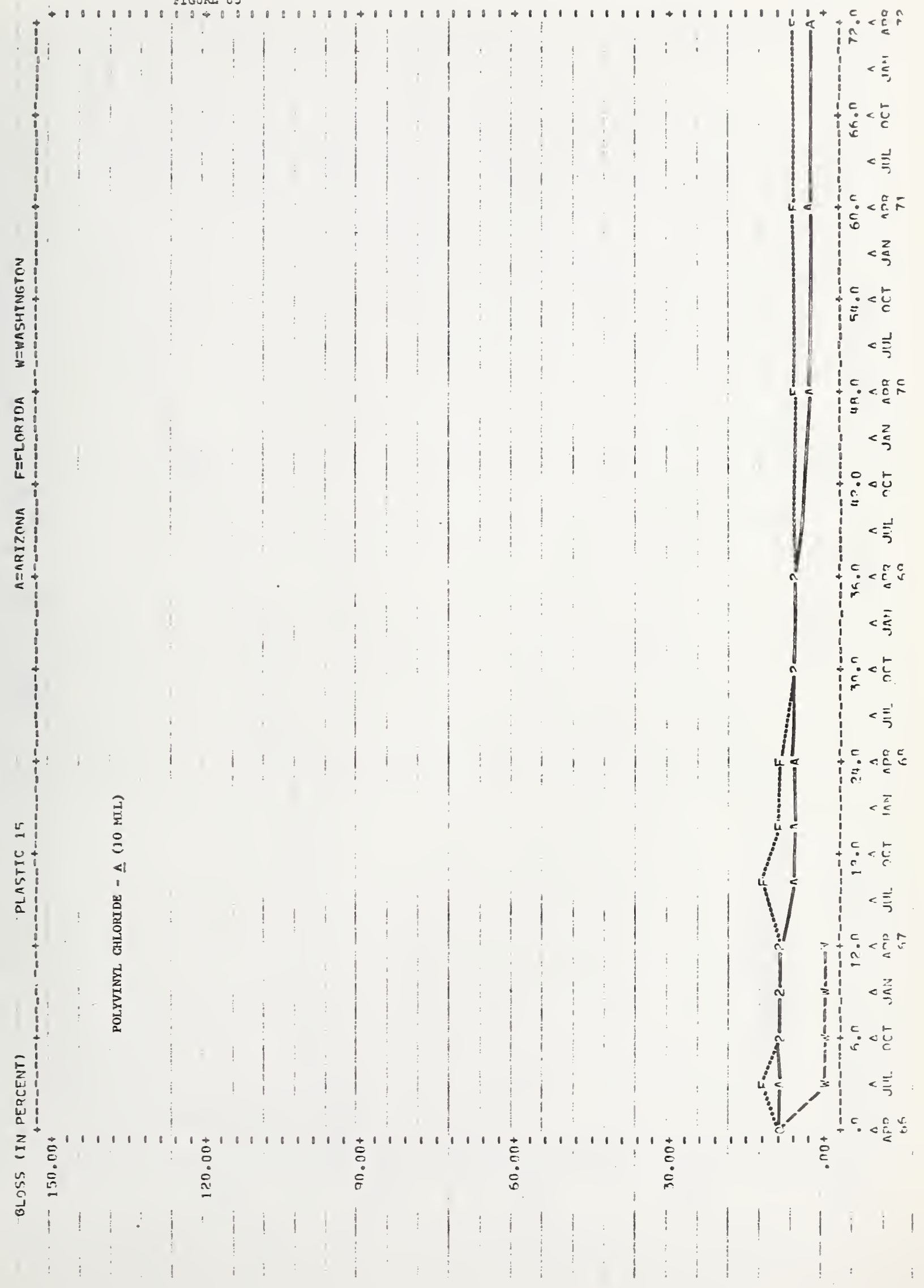


FIGURE 64

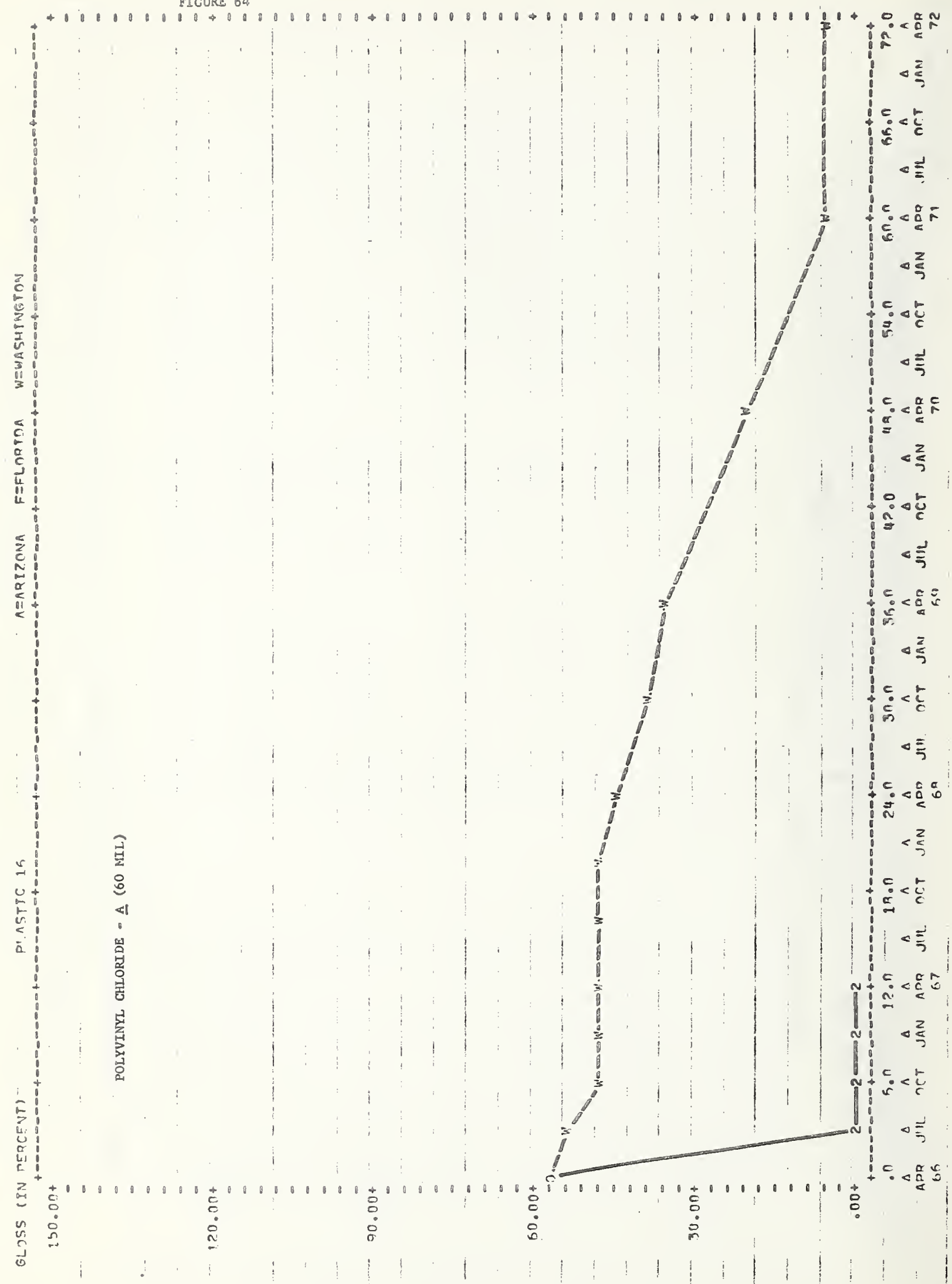


FIGURE 65

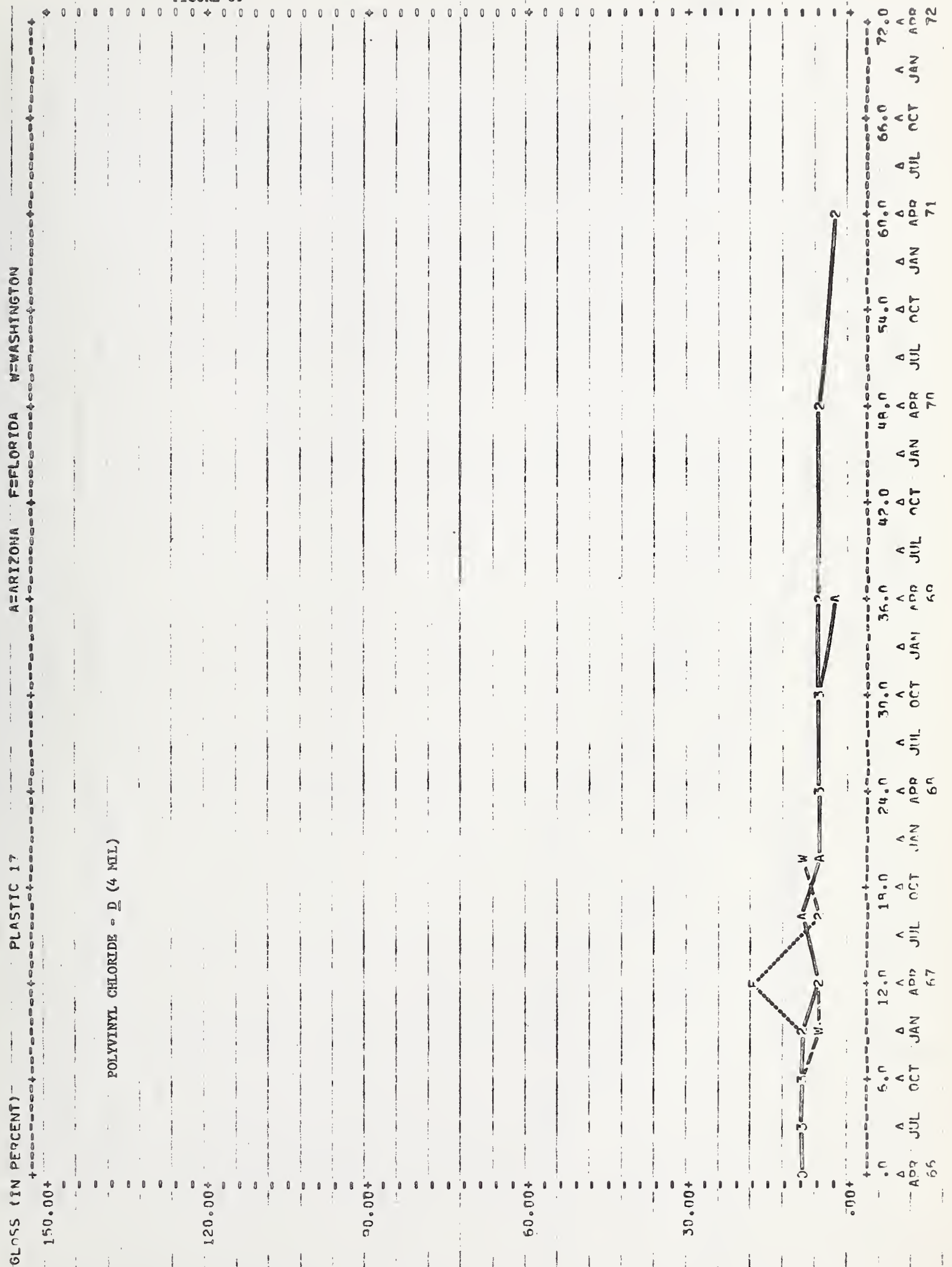


EICURE 66

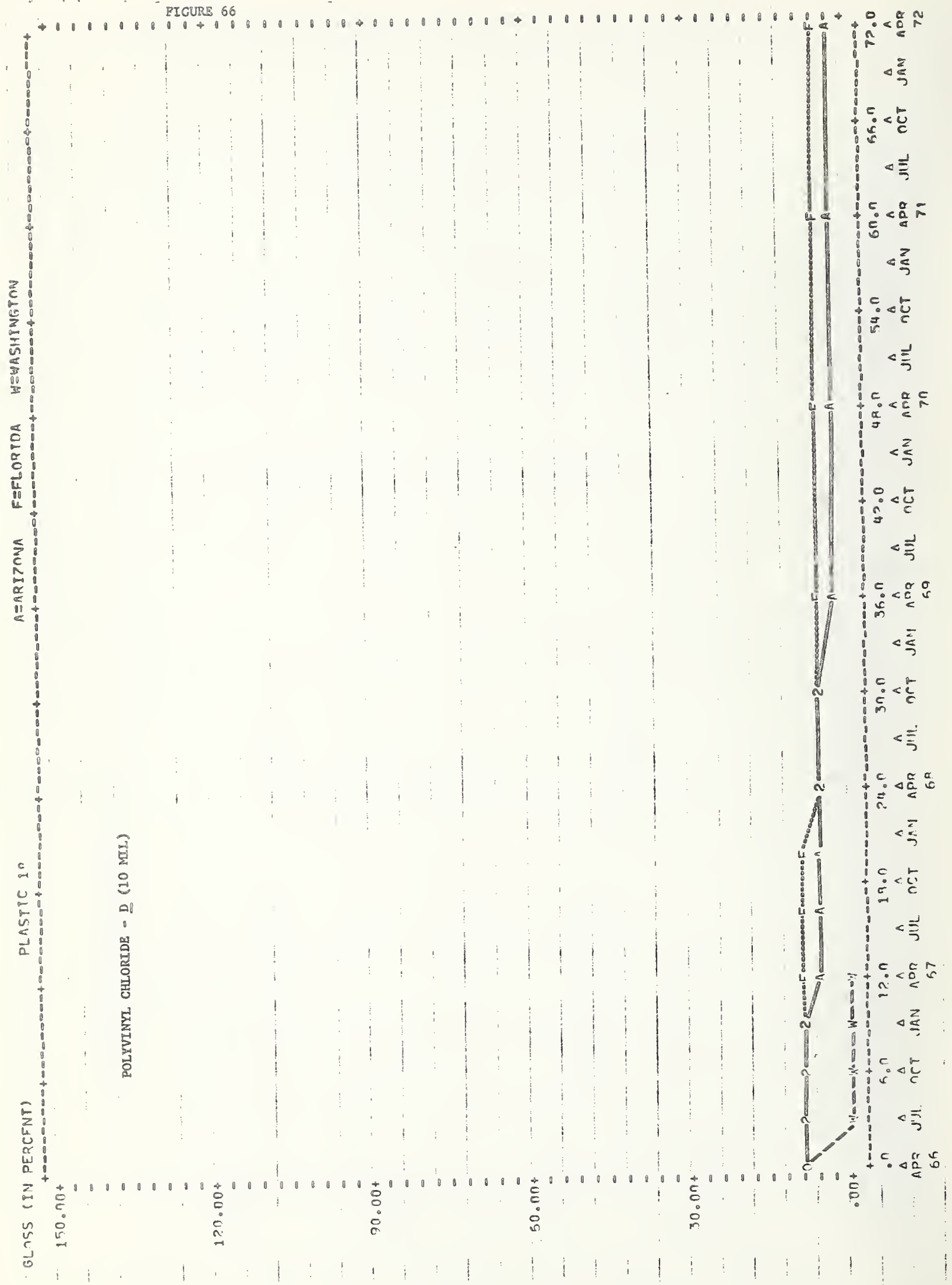




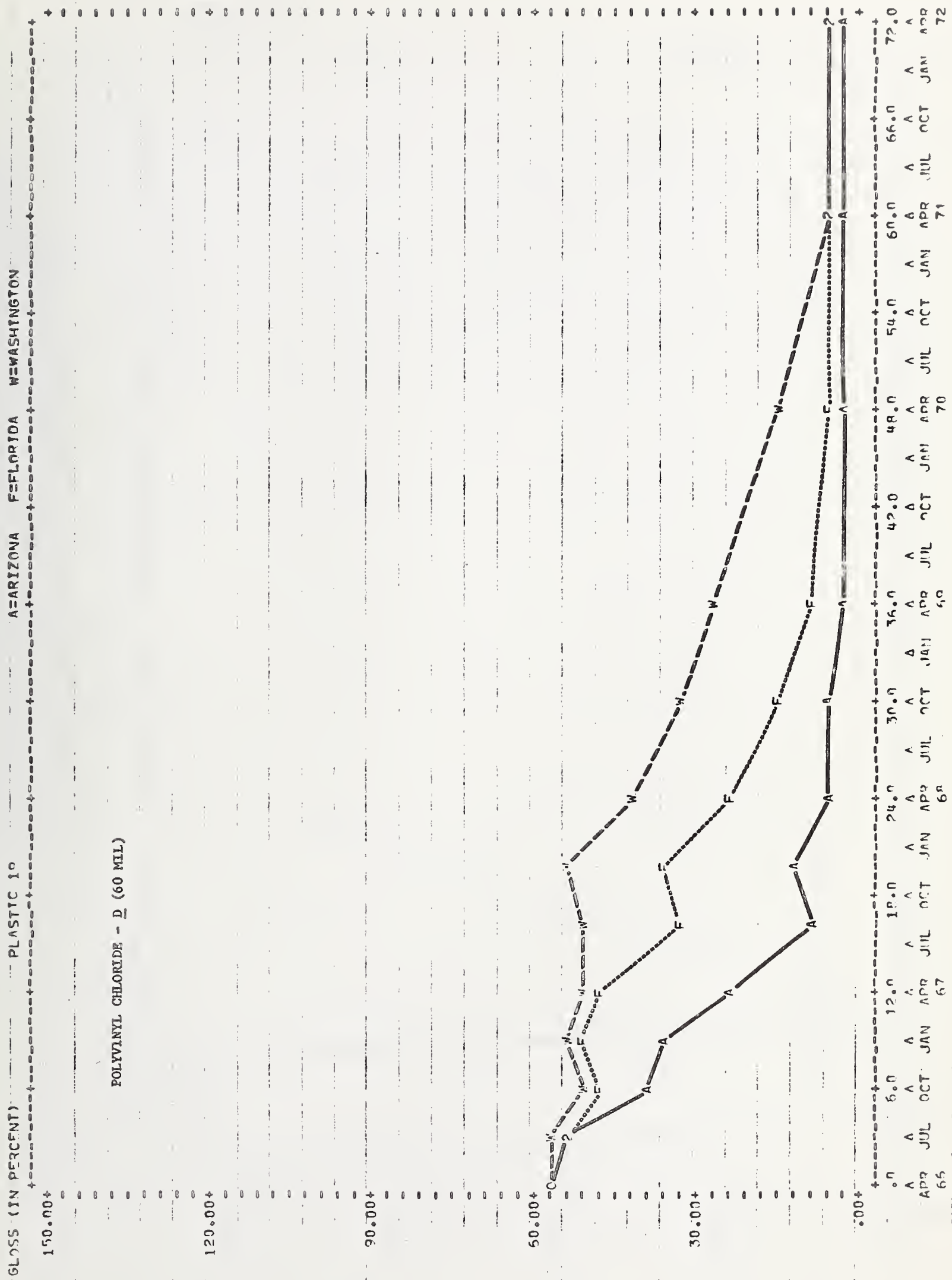


HIGUIK 68

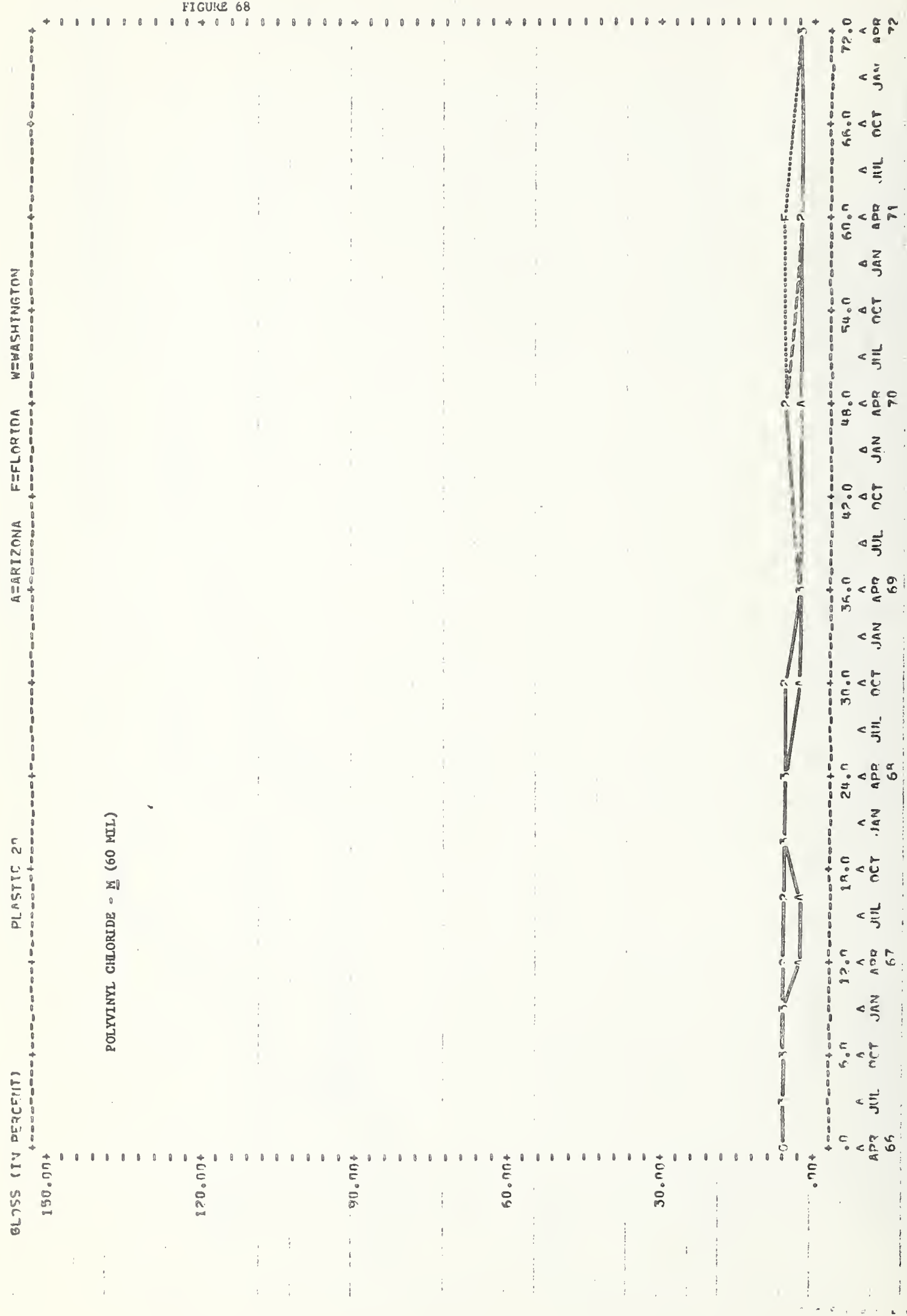


FIGURE $69 \mathrm{~A}$

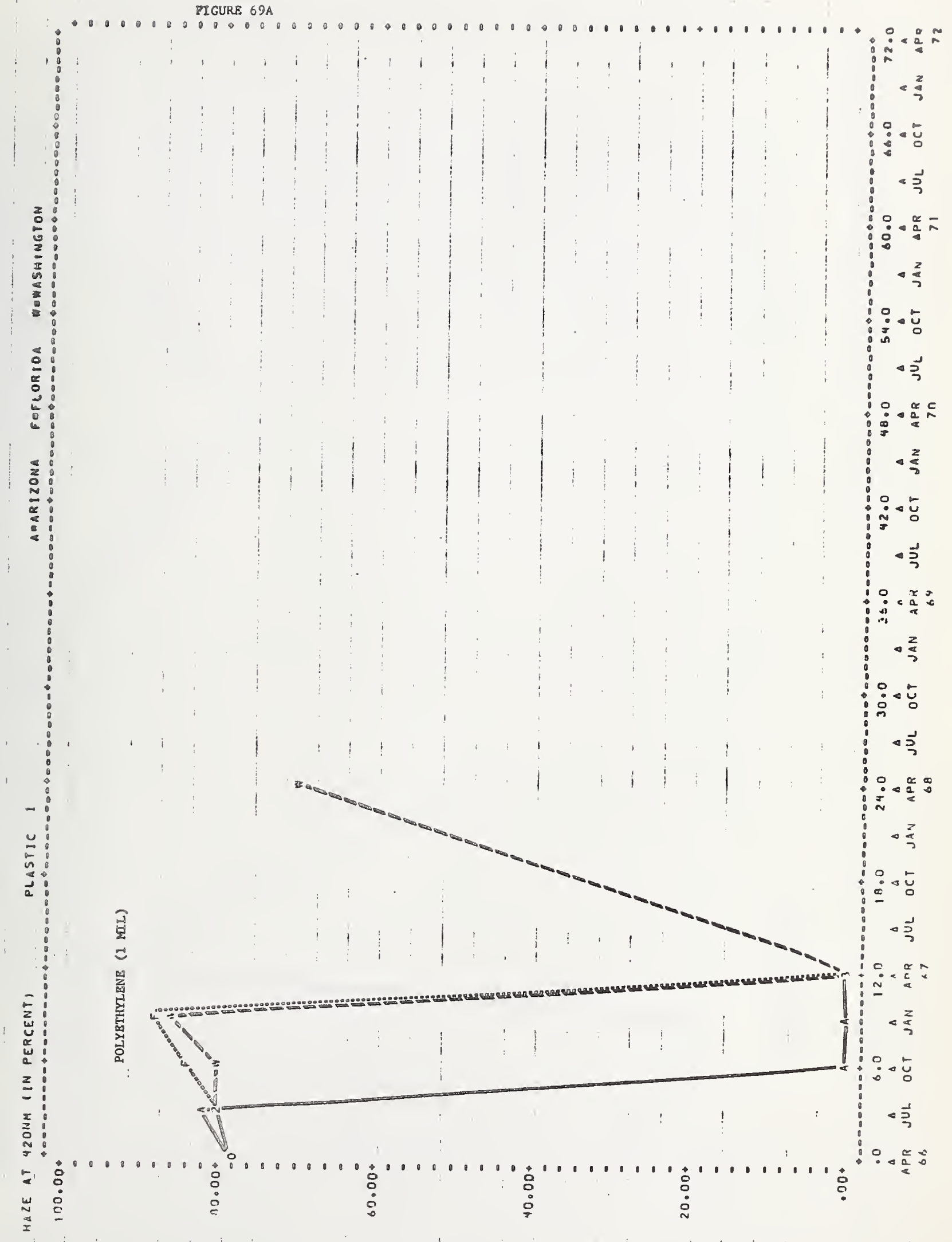


PICURE 69B

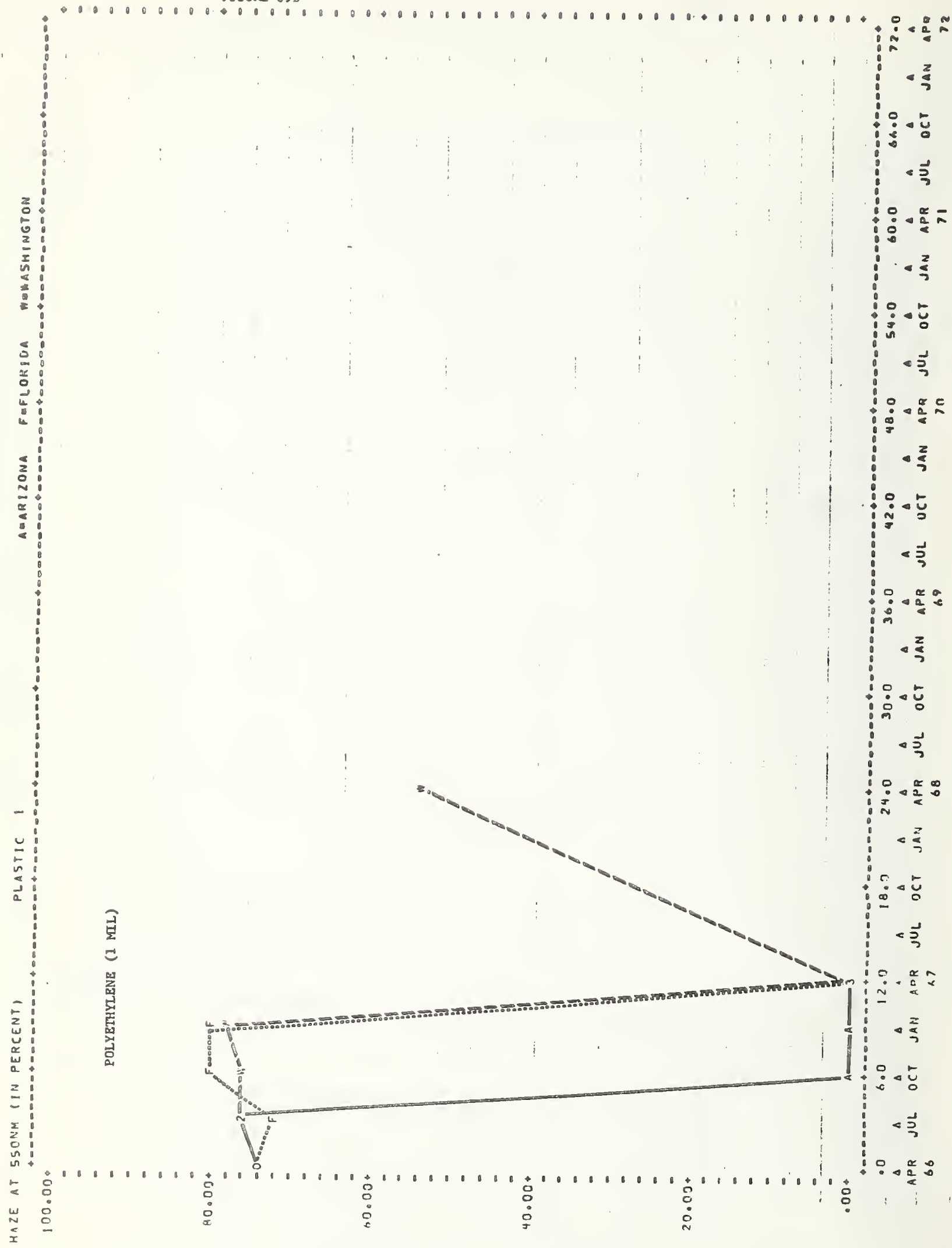




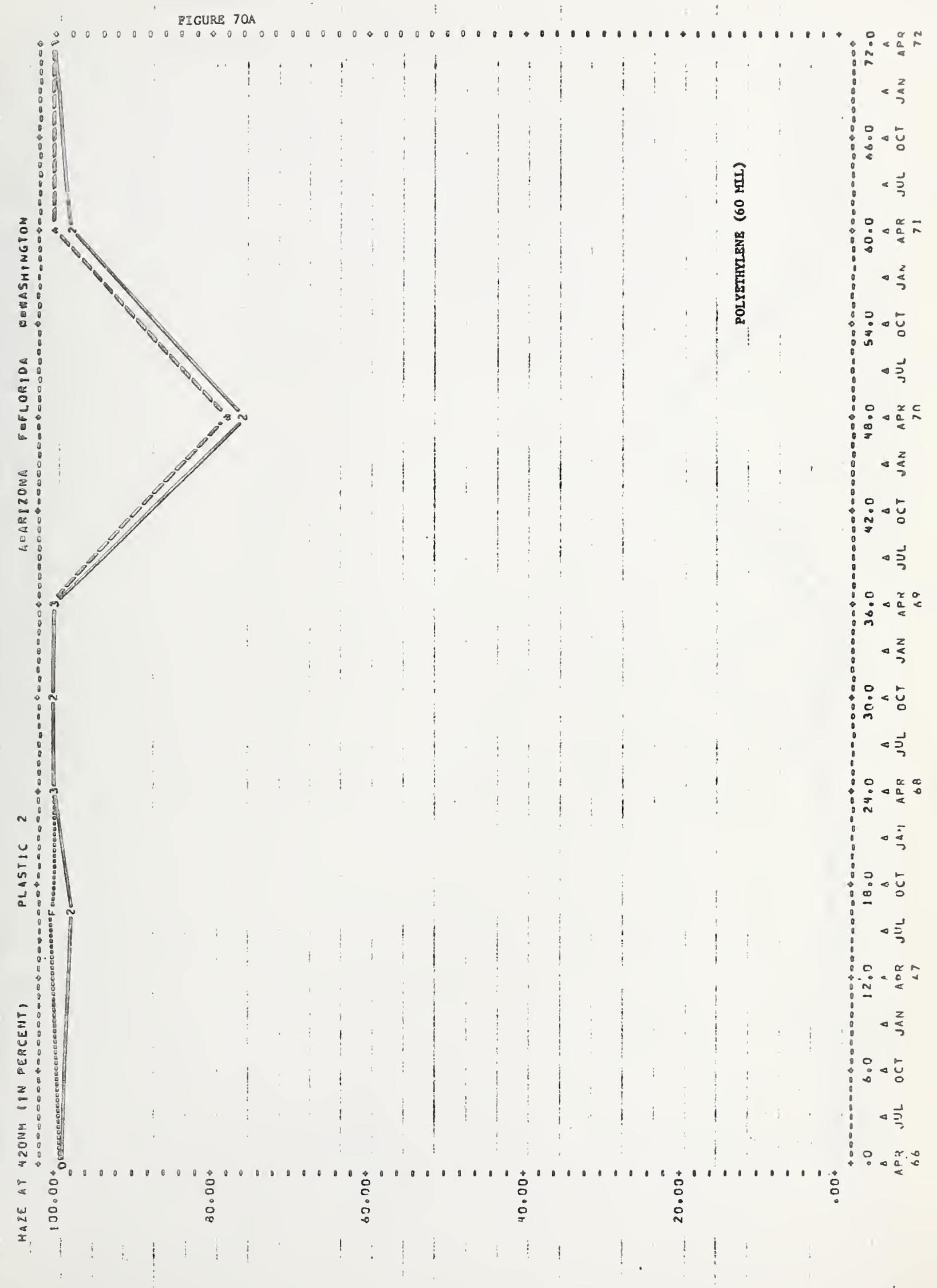


FIGURE $70 \mathrm{~B}$

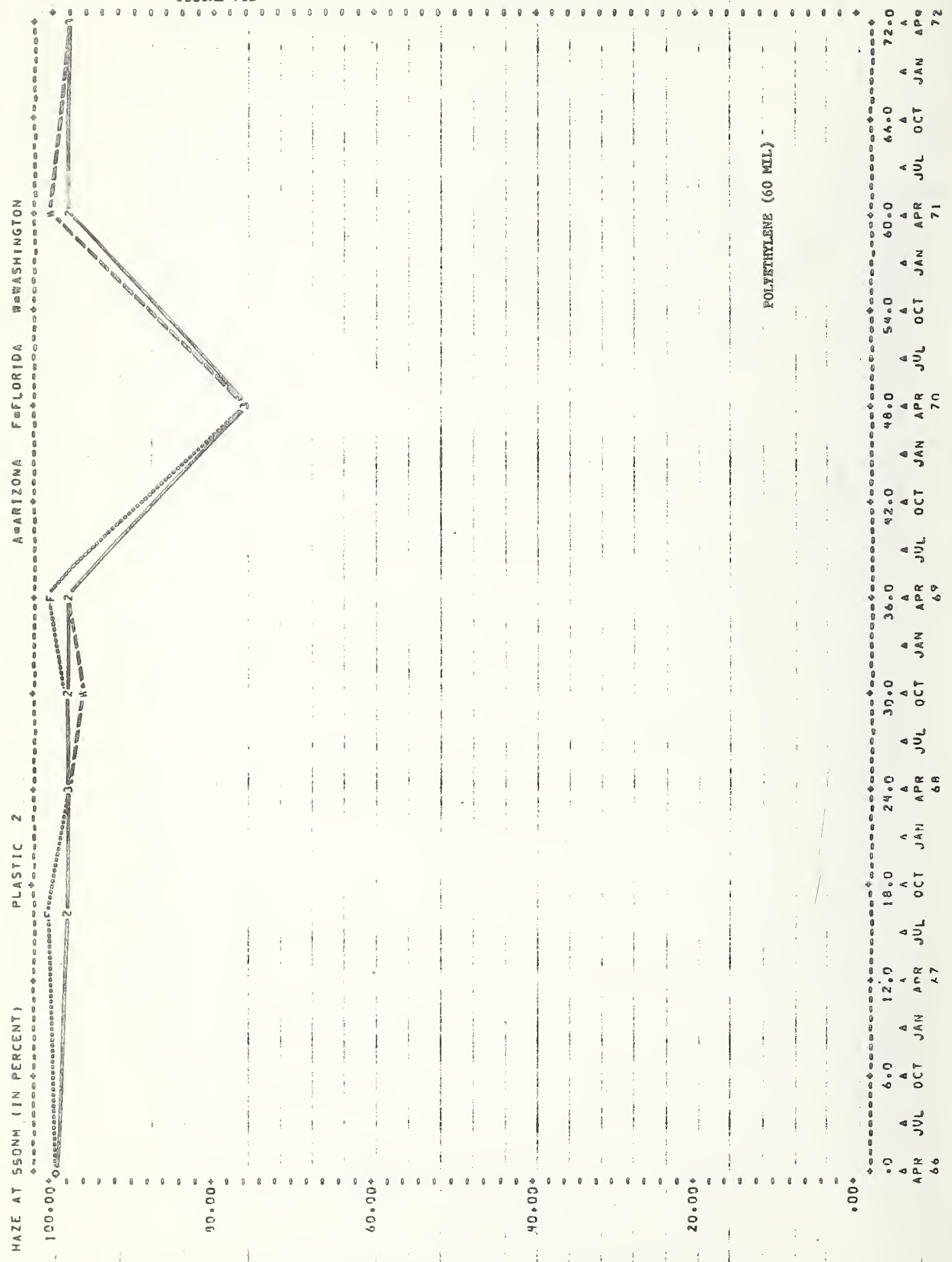




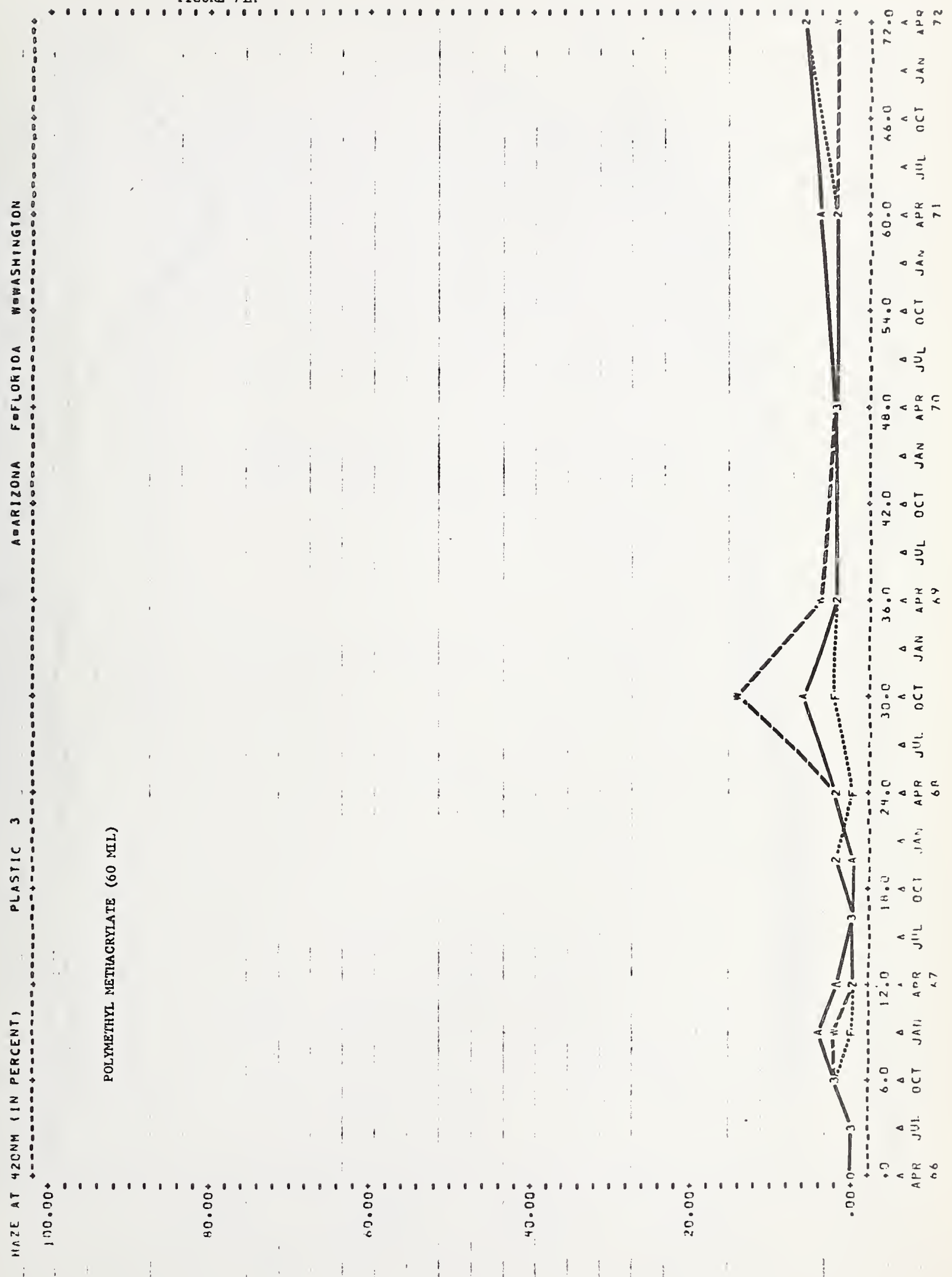


FIGURE 71B

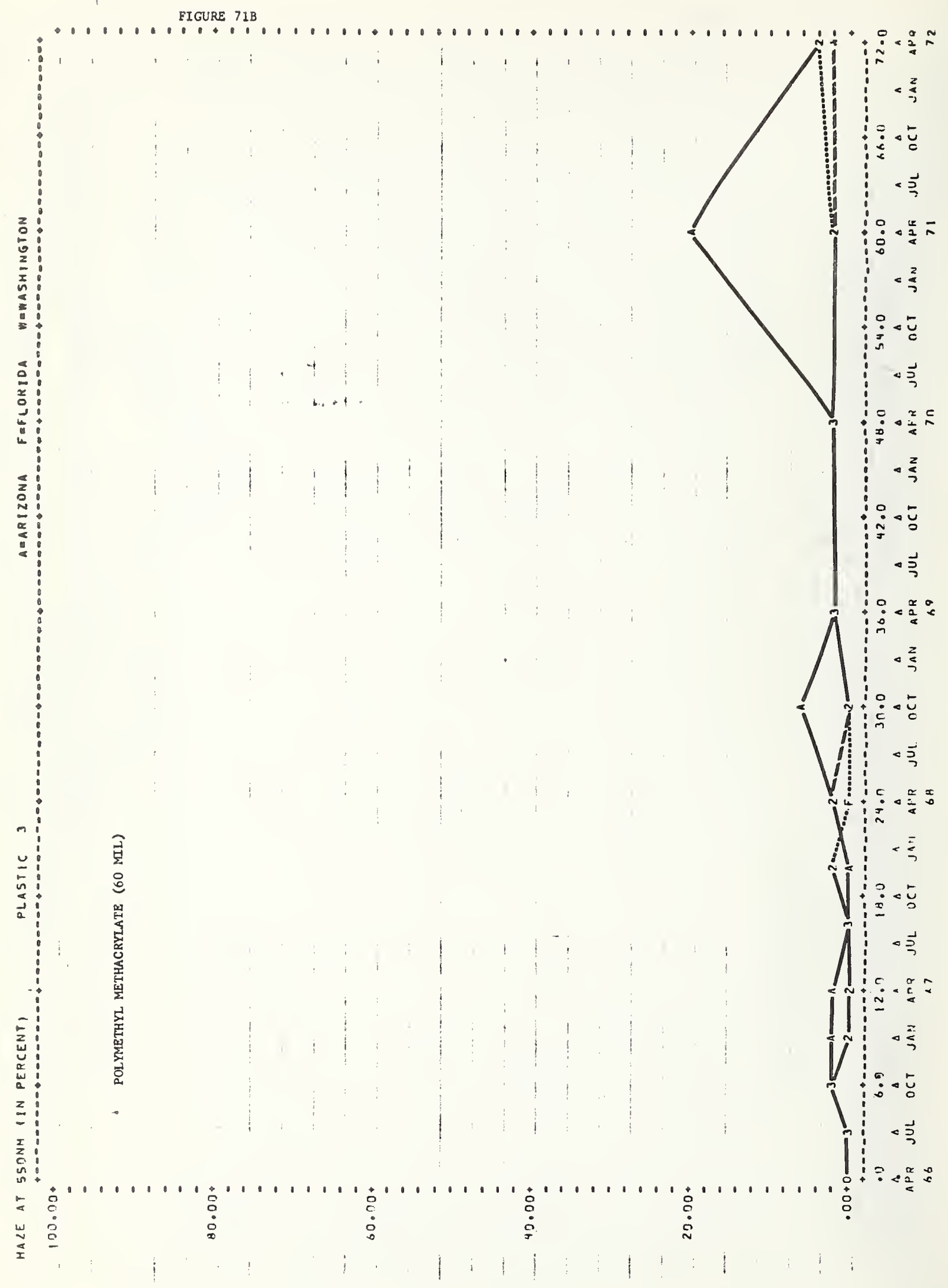




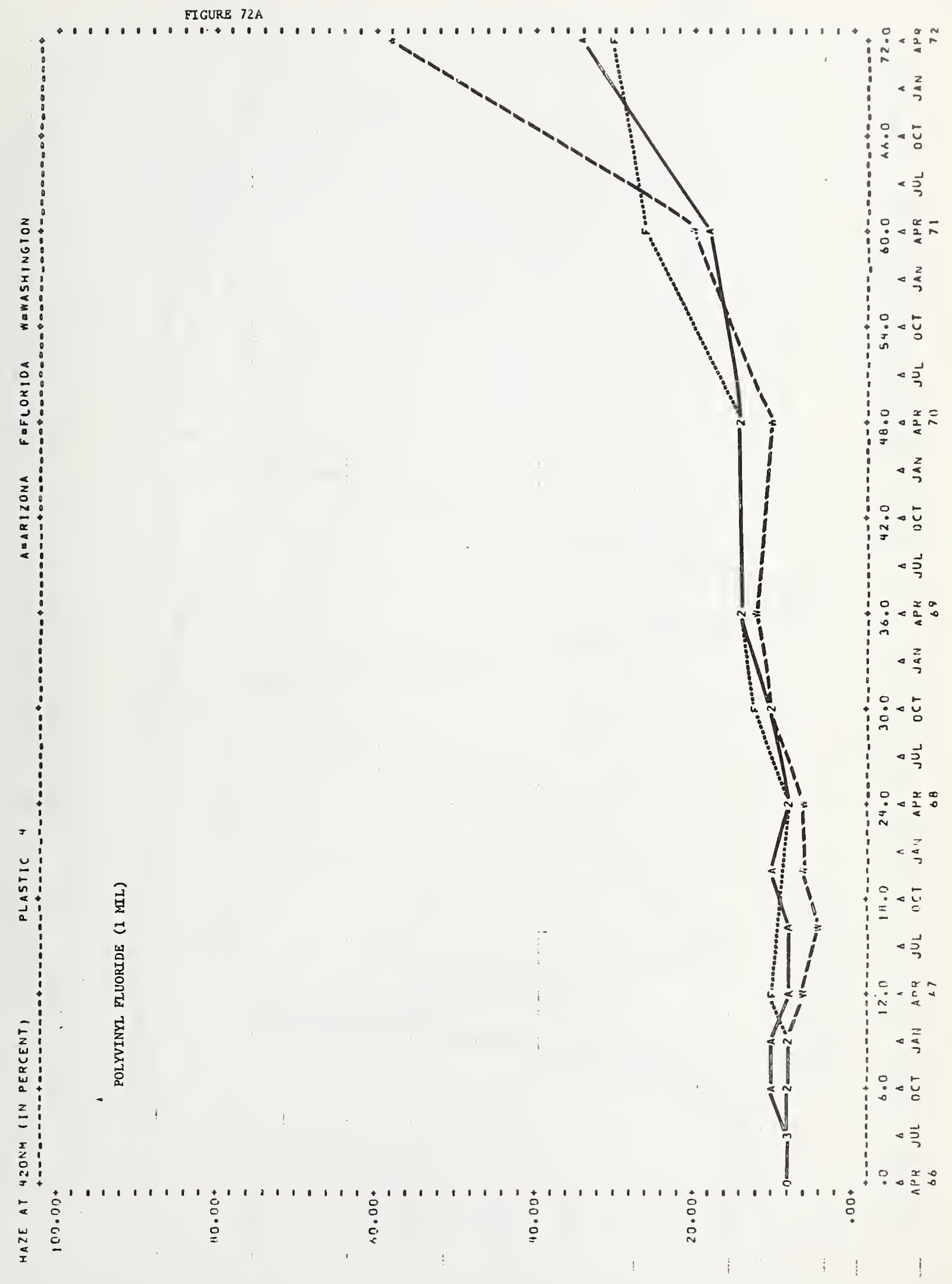


PIGURS $72 \mathrm{~B}$

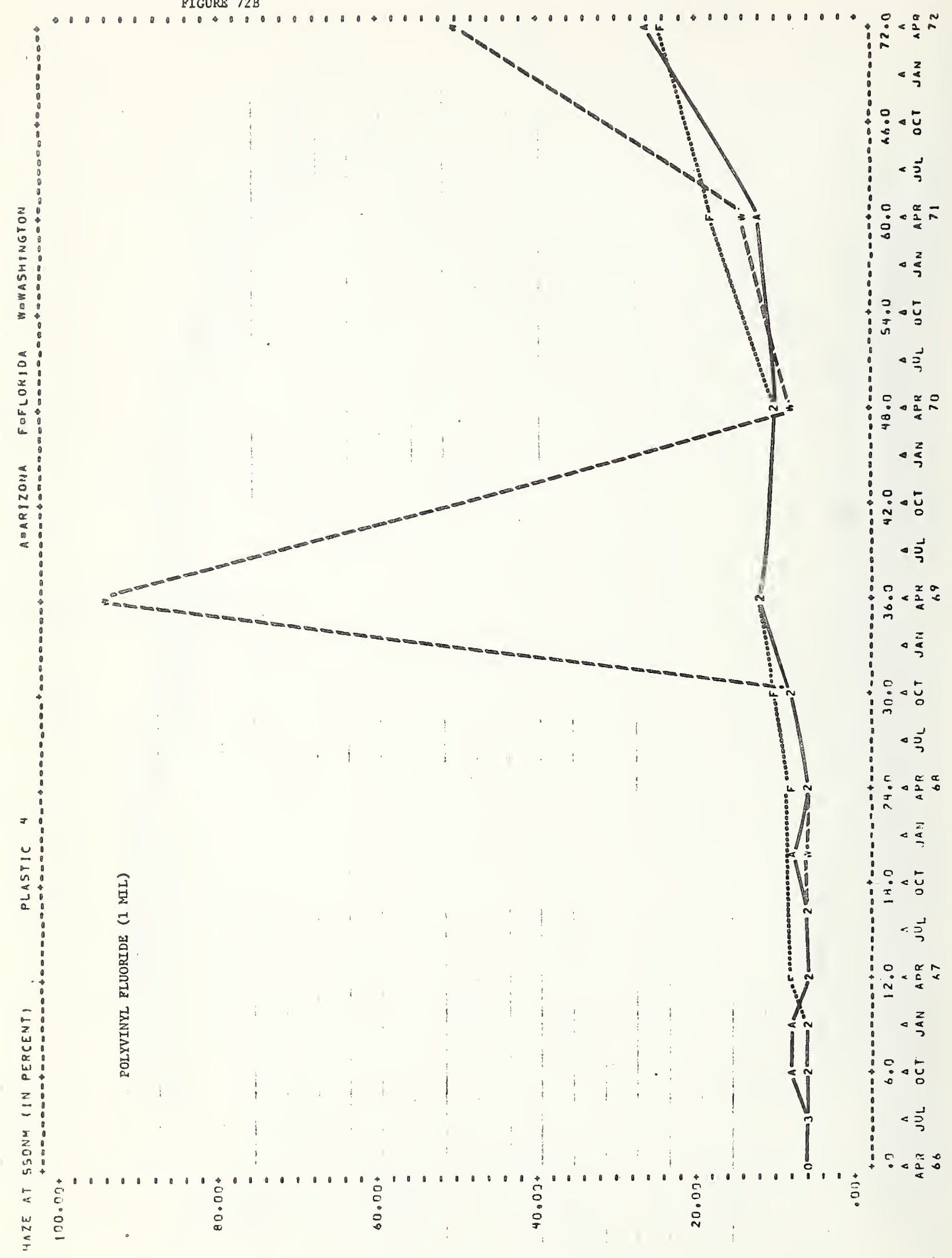




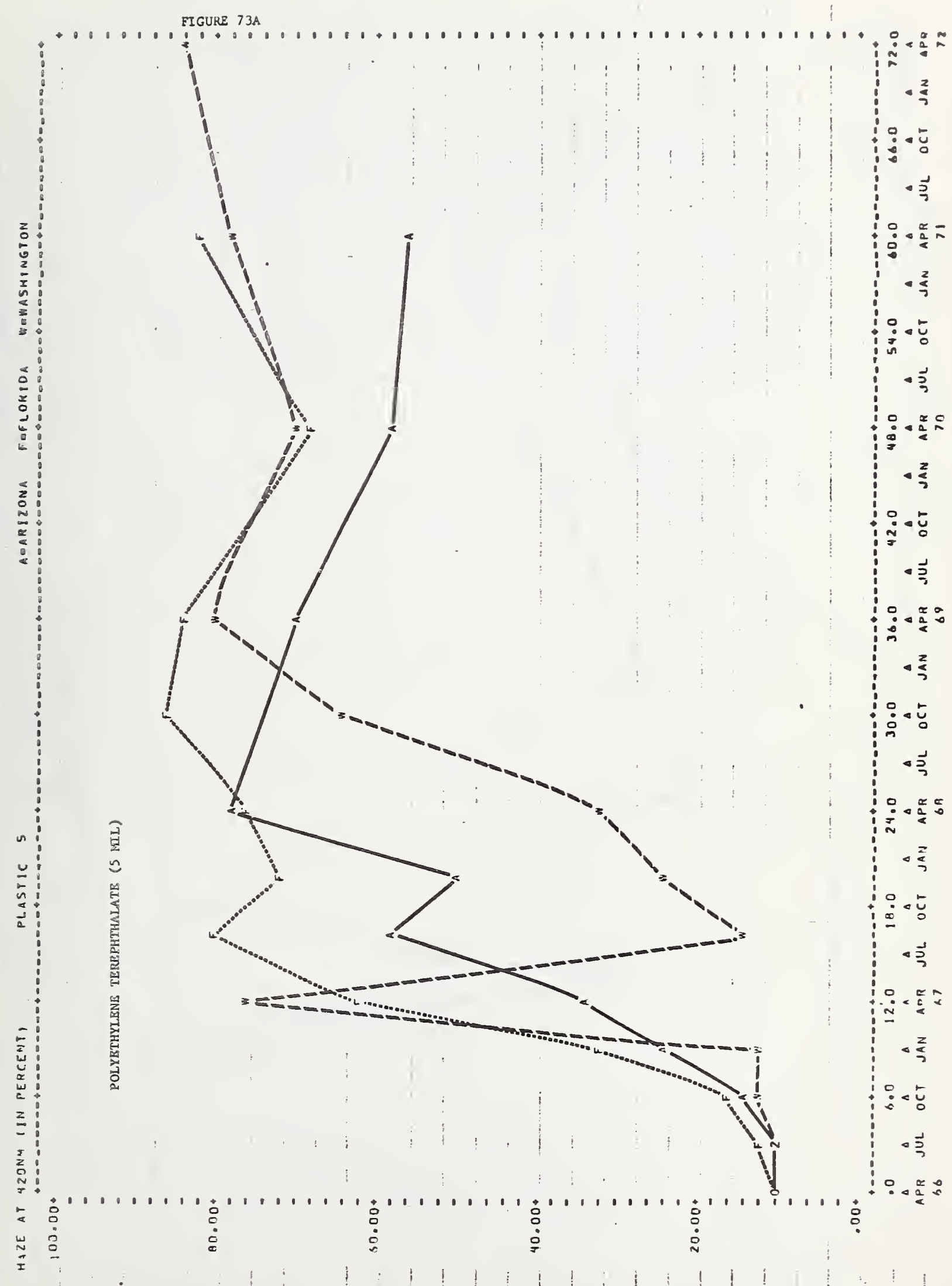


EIGURE 73B

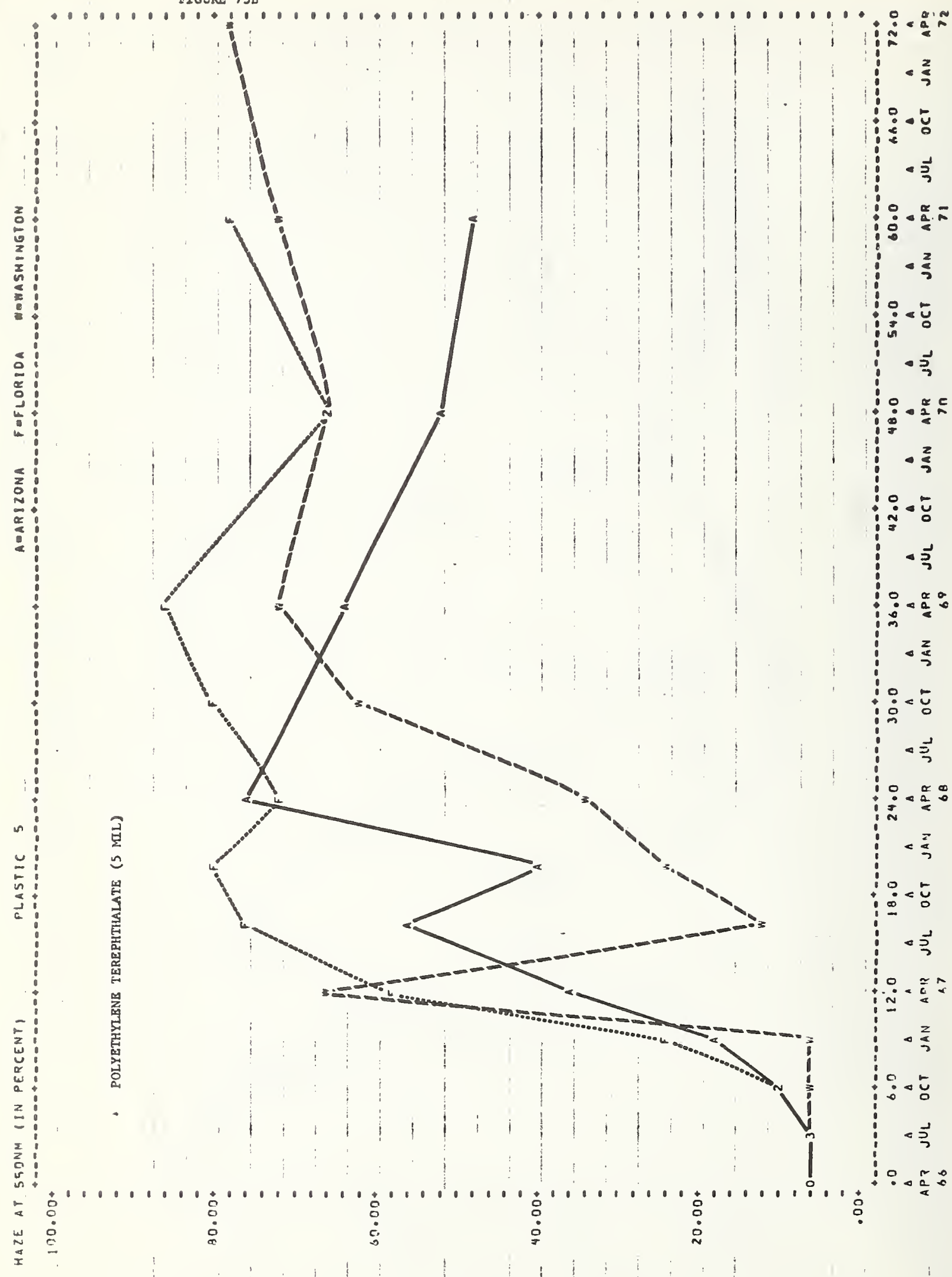


FIGURR $74 A$

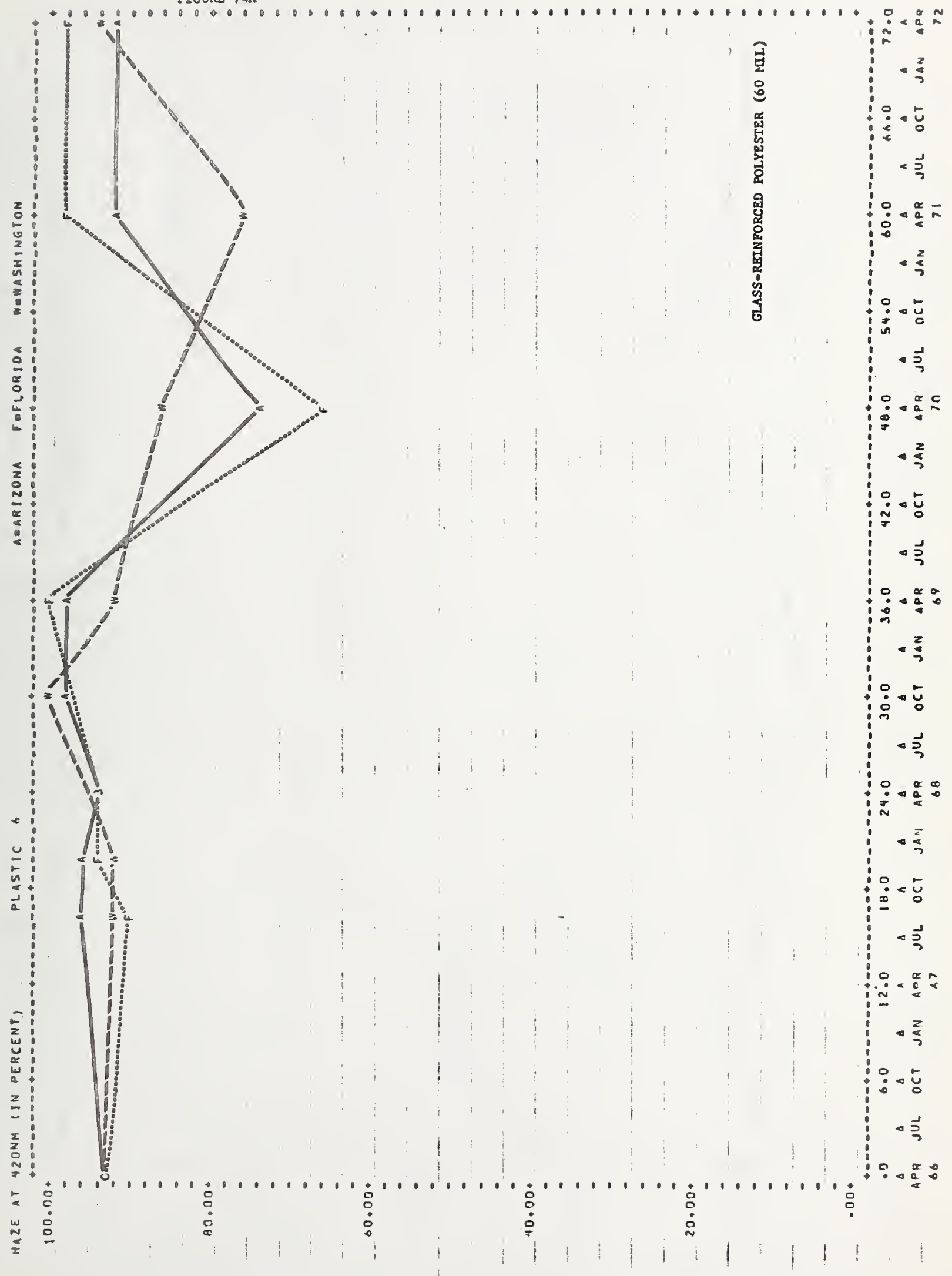




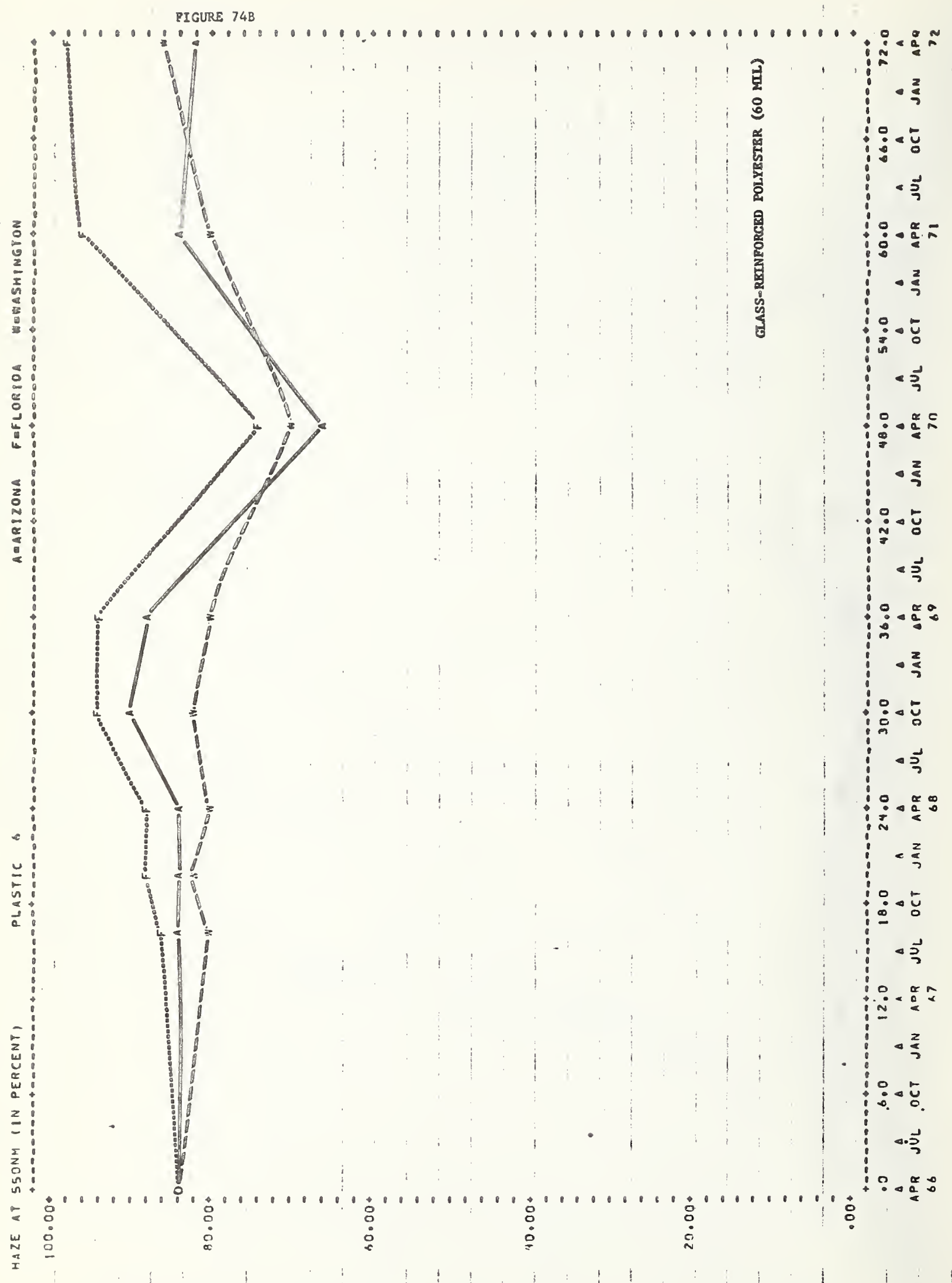




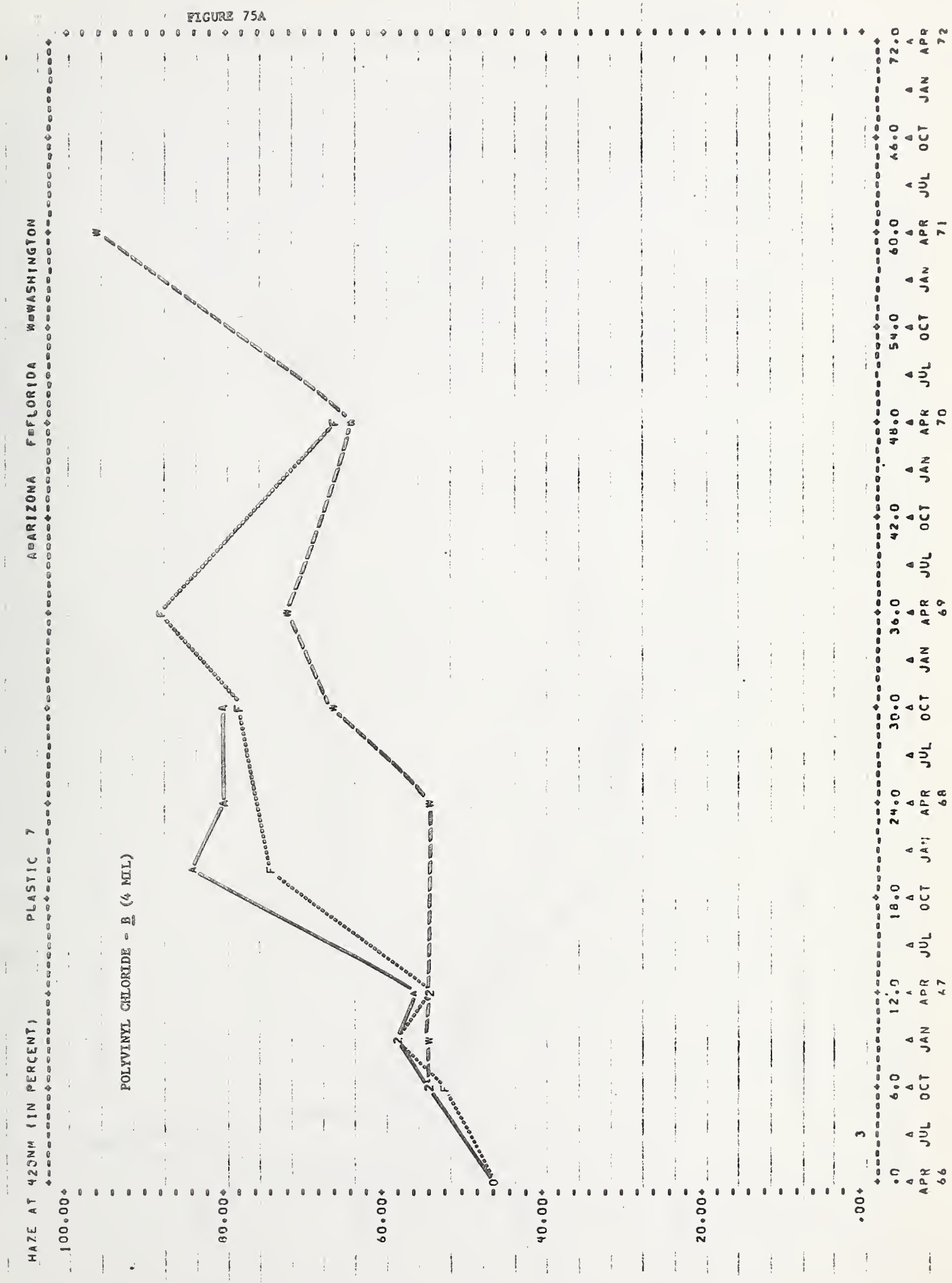




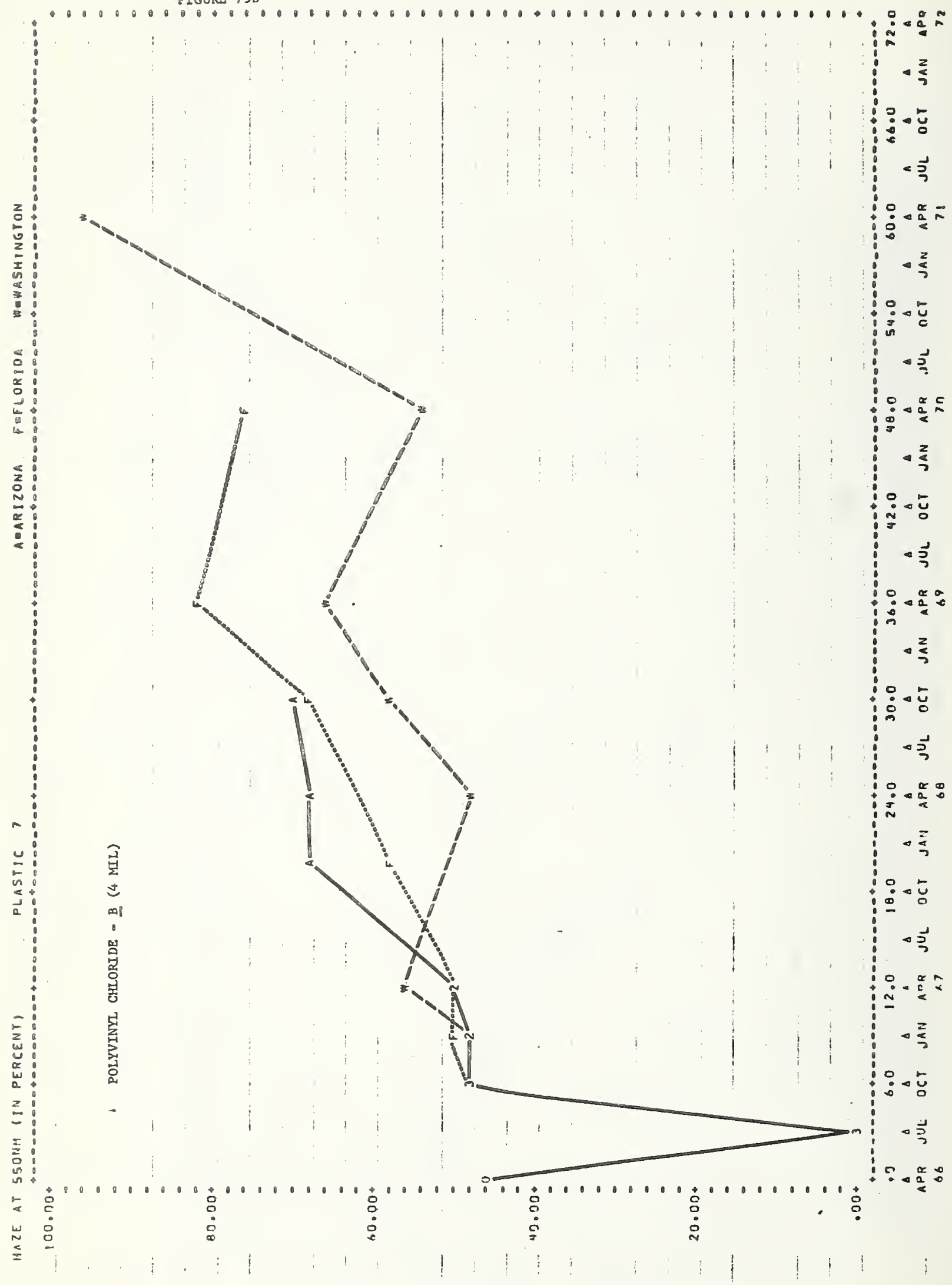




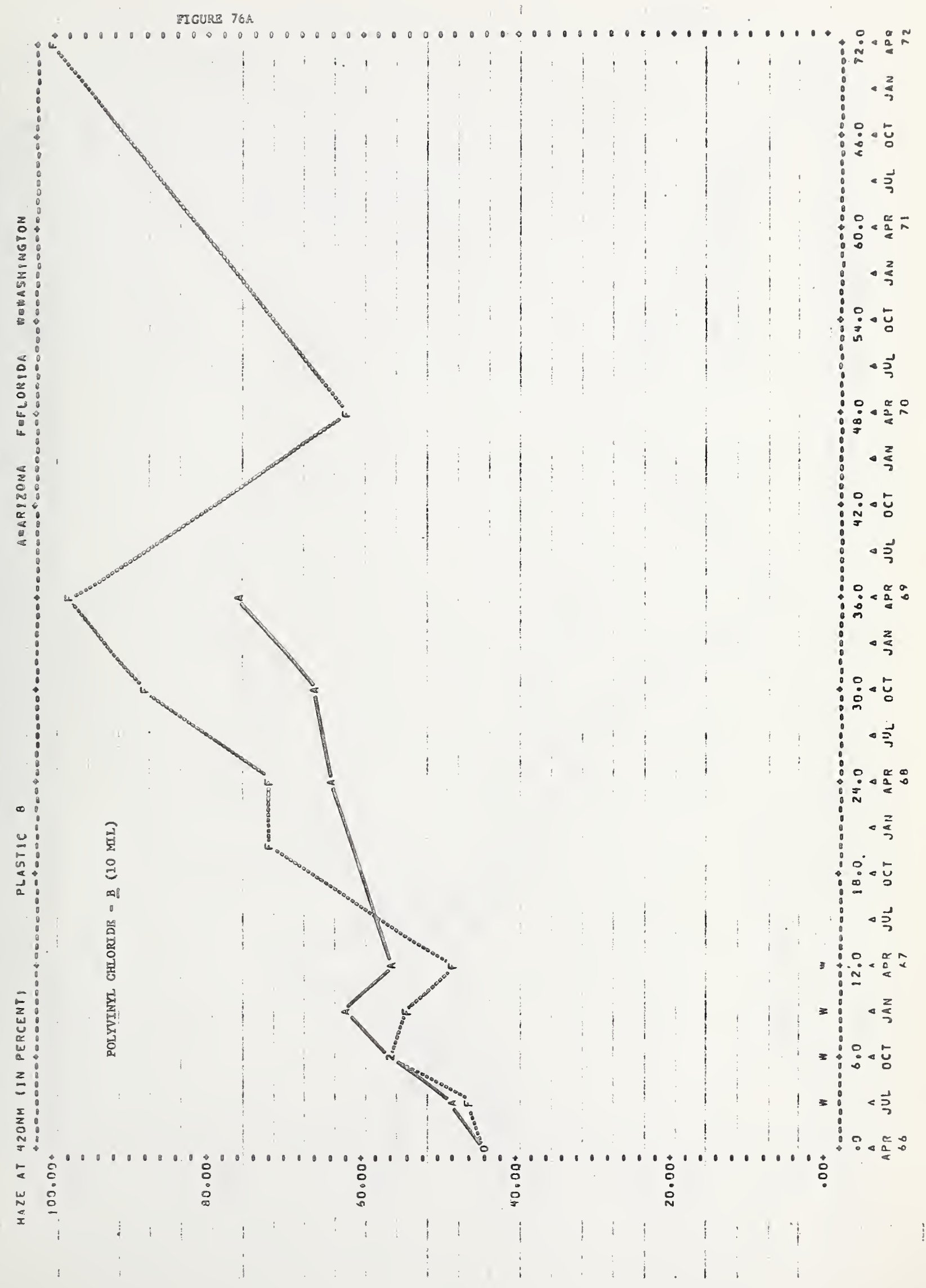


23

FIGURE 768

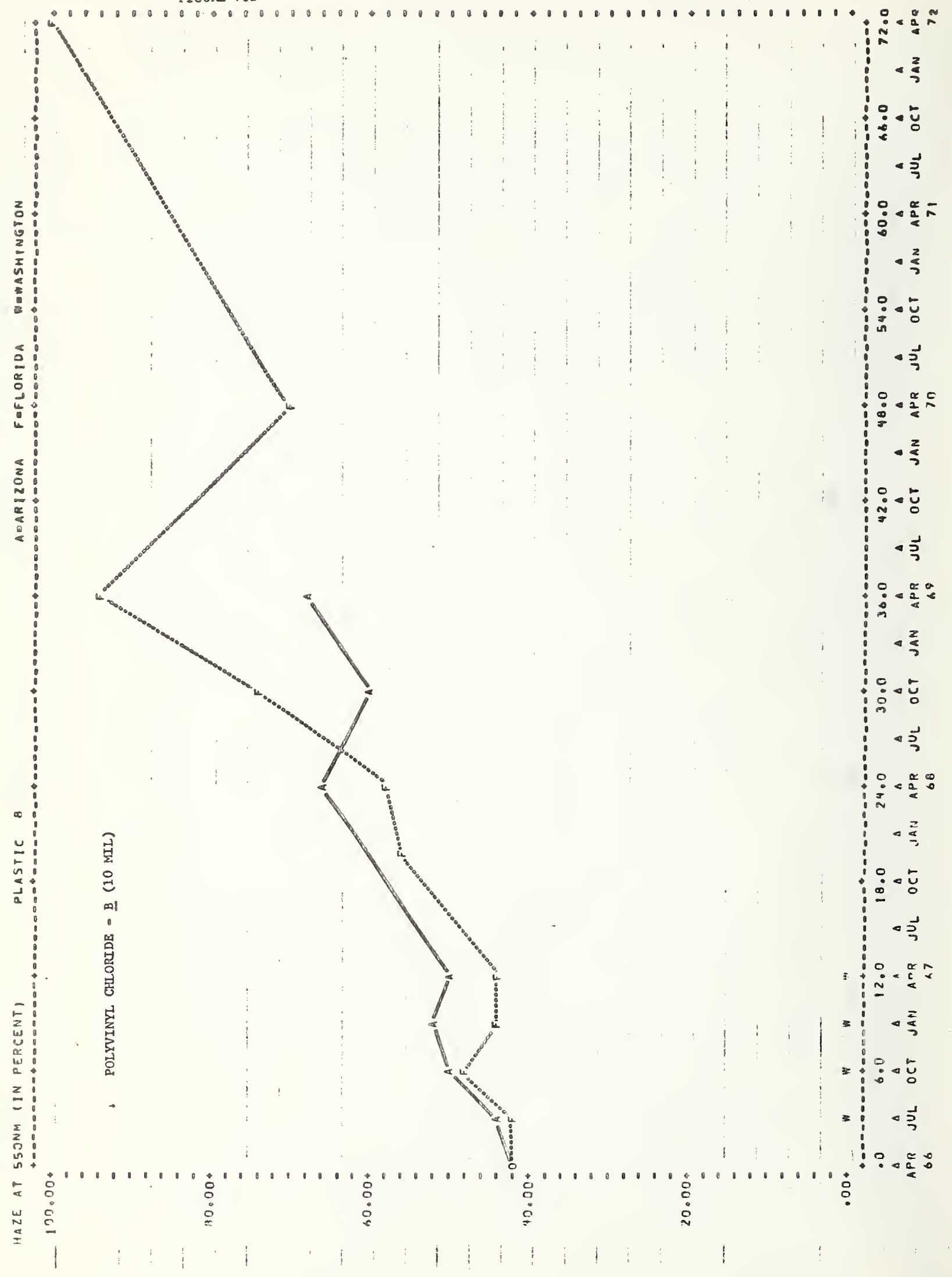




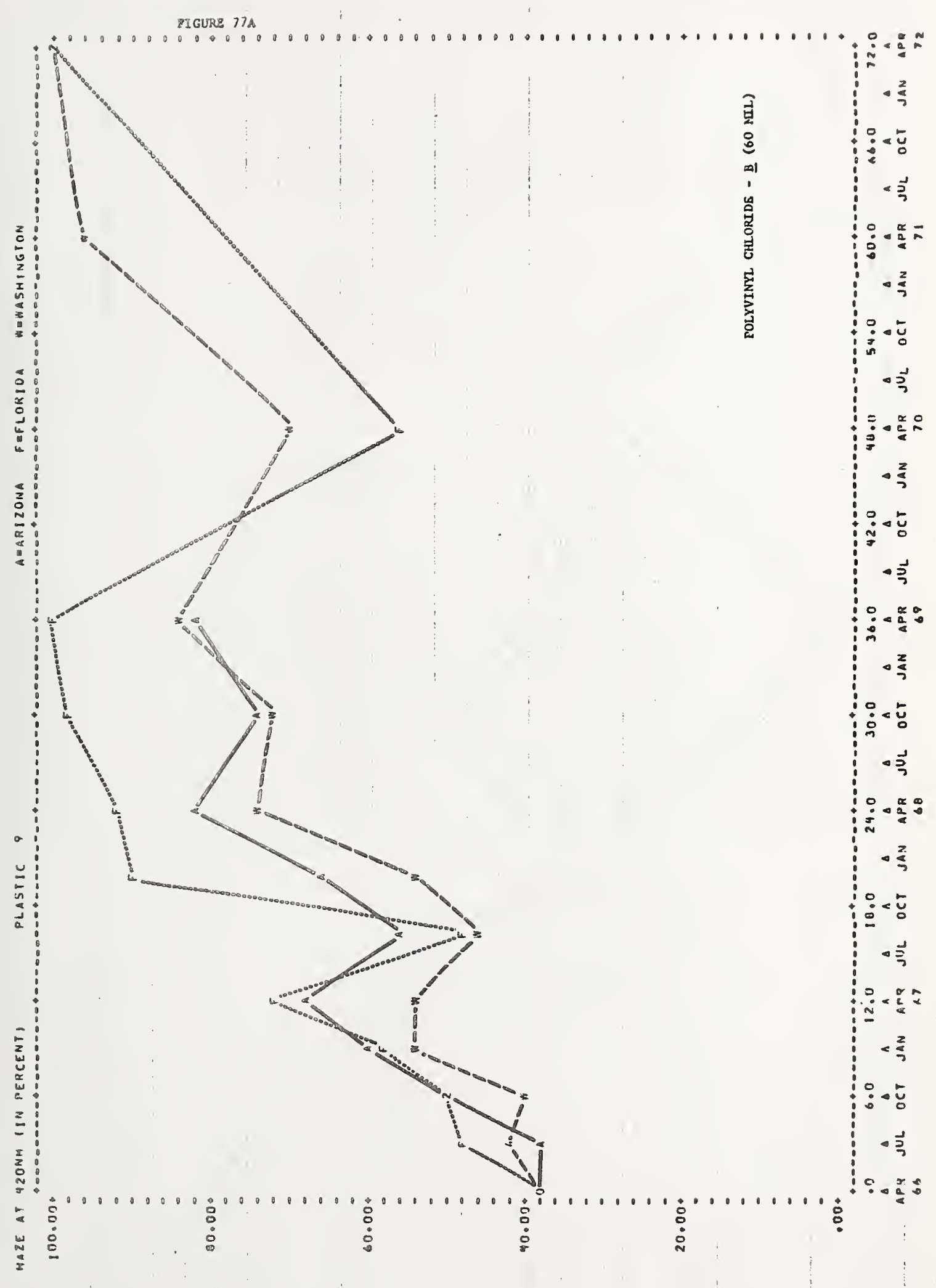




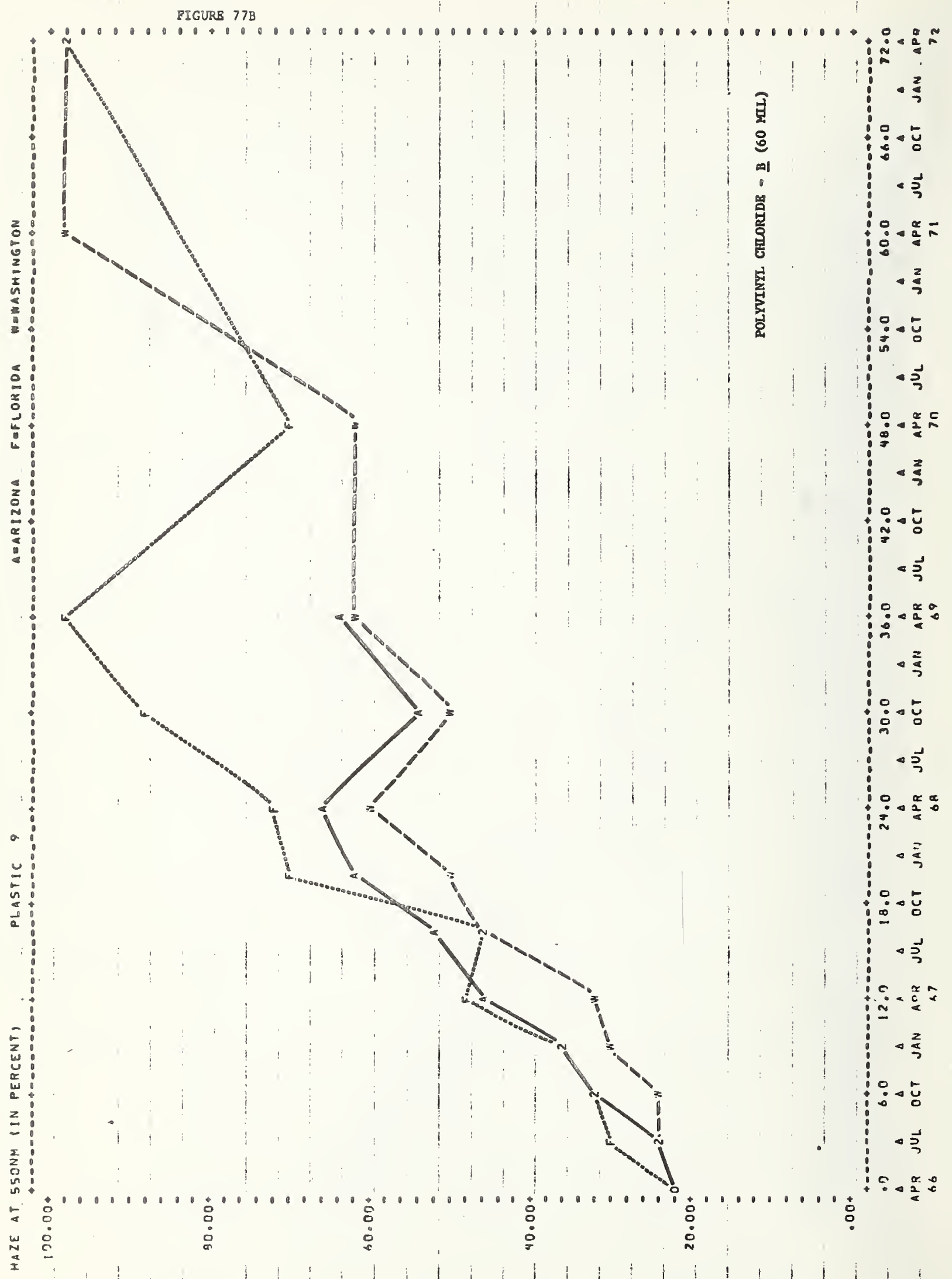


RIGURE TA

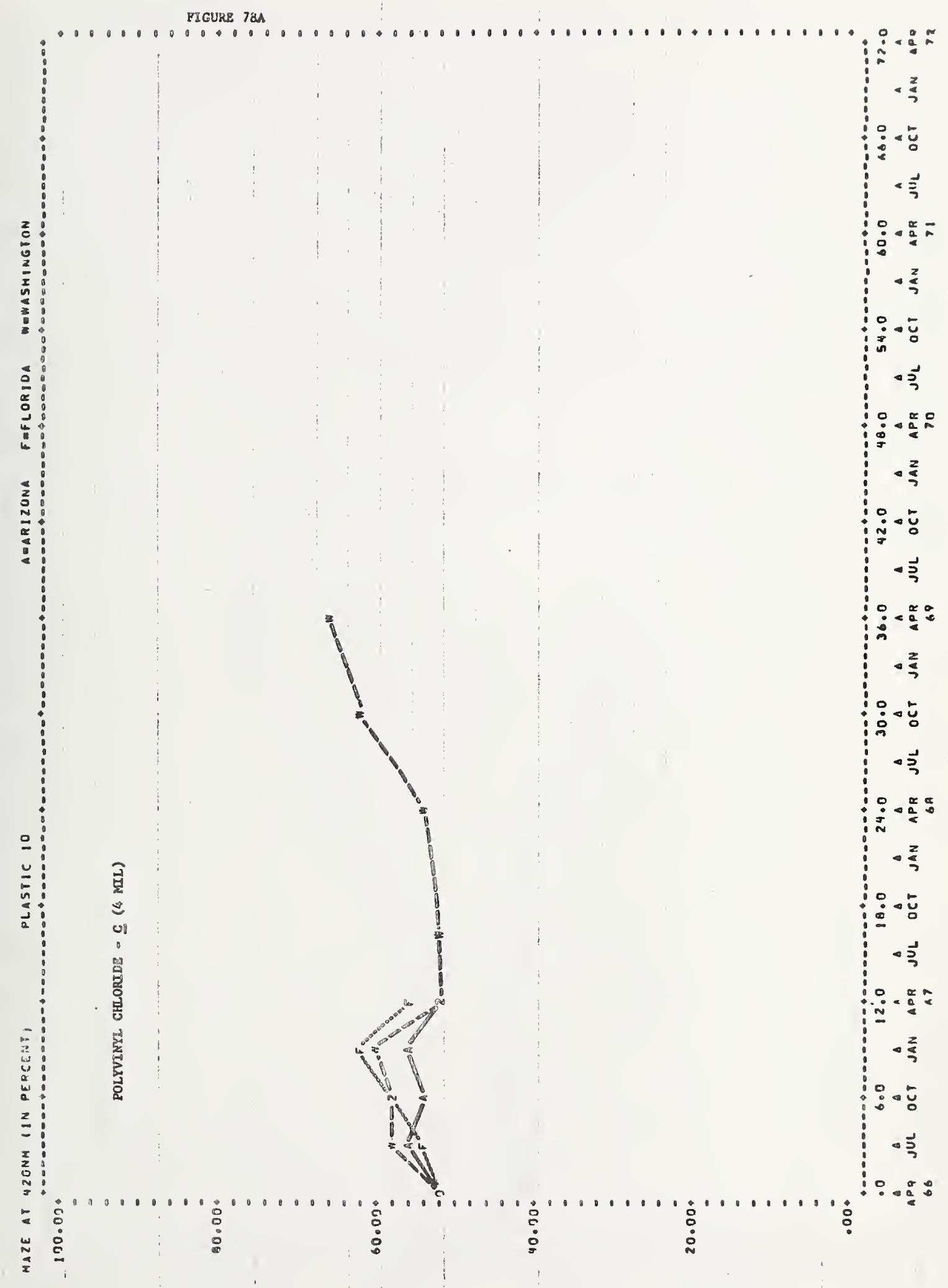


EICURE 78B

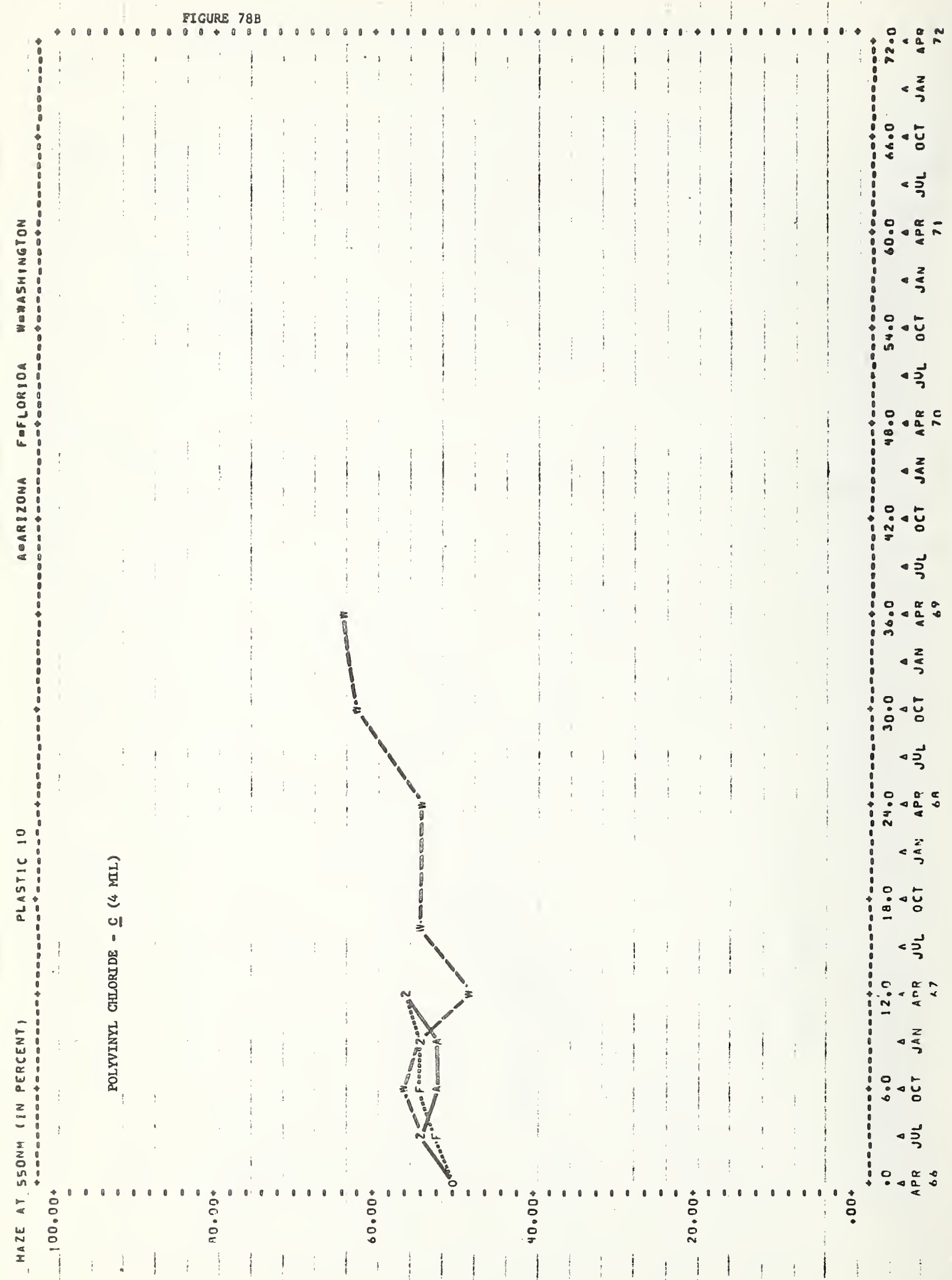


RIGURE $79 A$

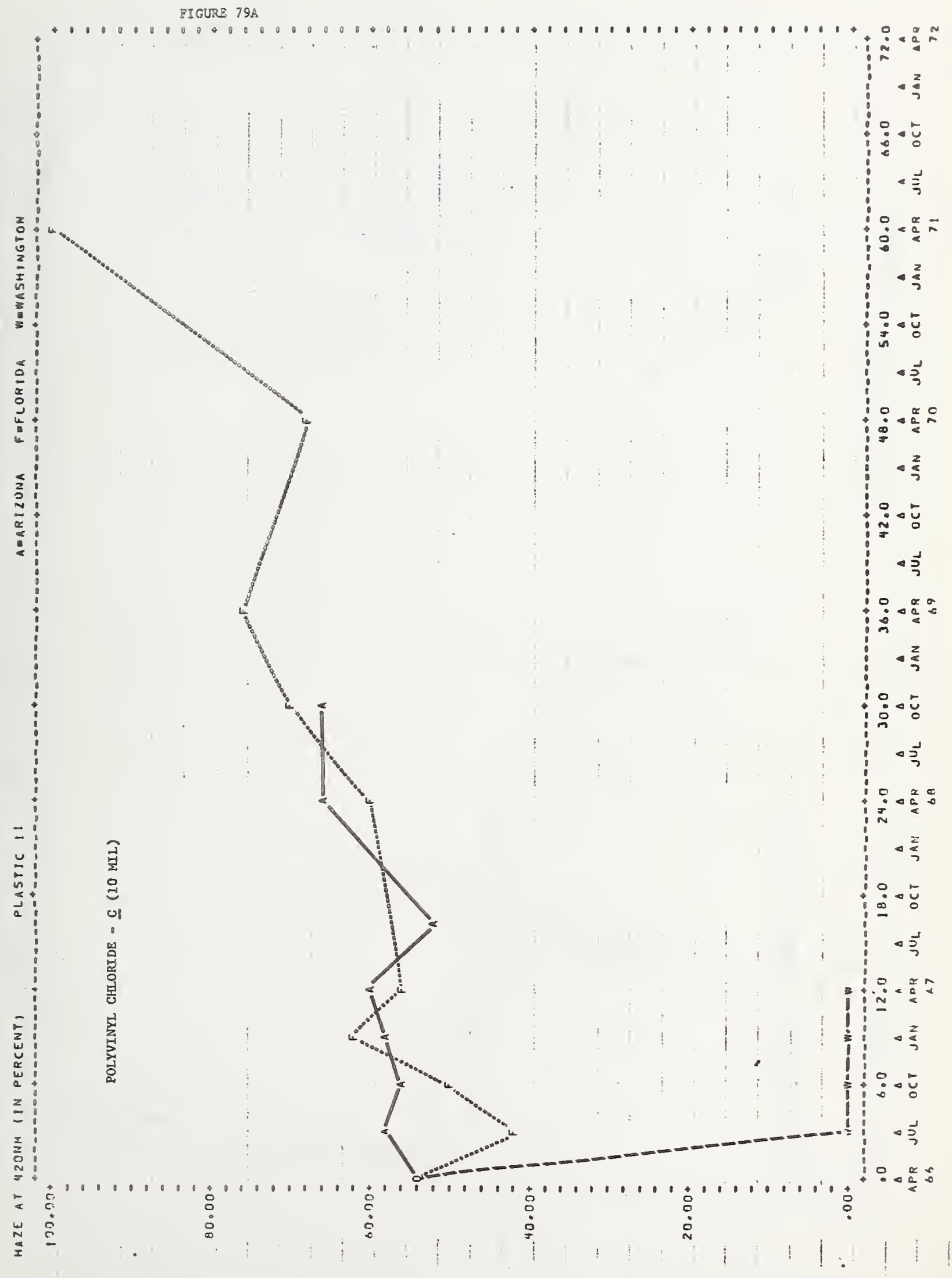




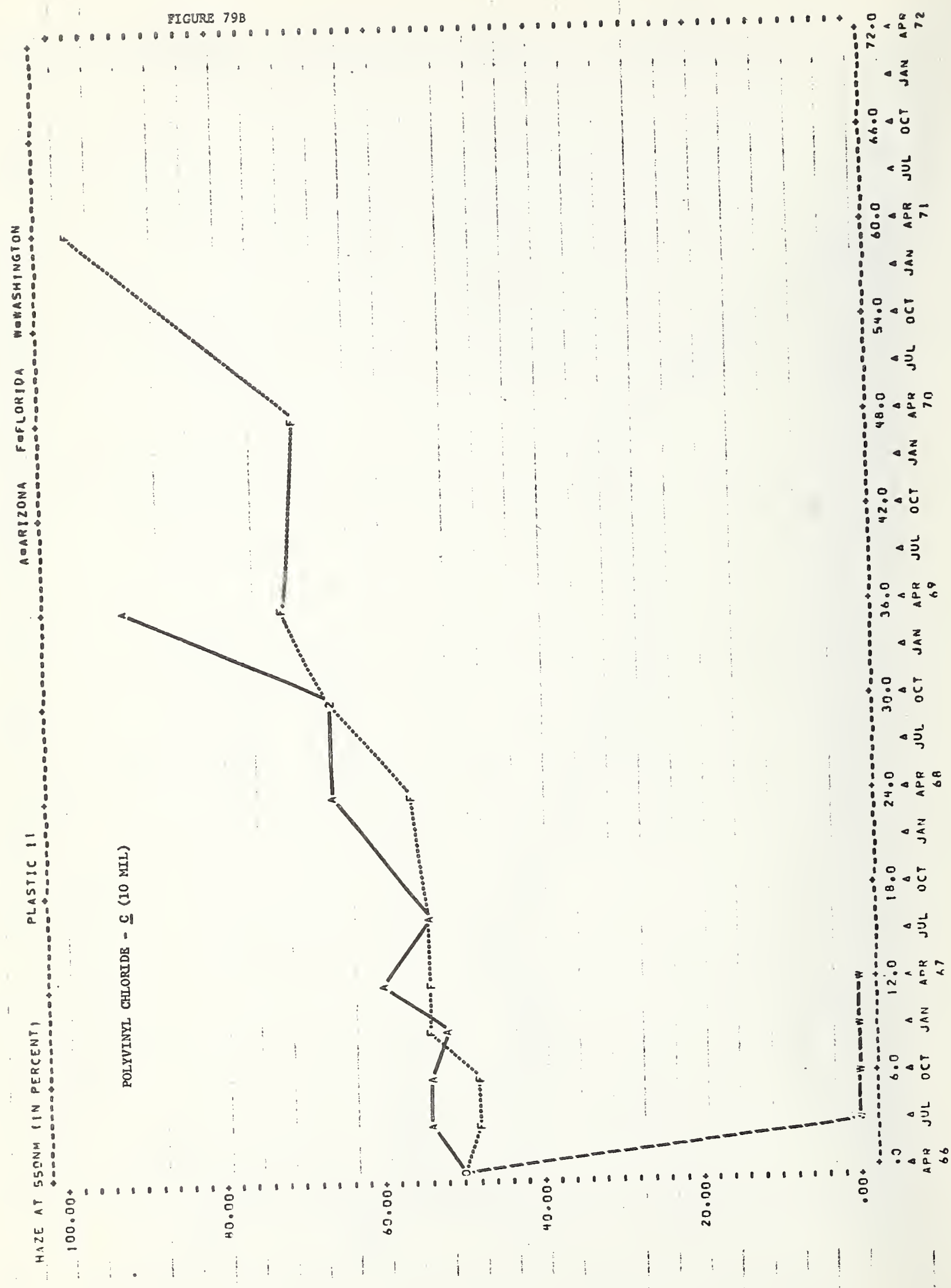


RIGURE BOA

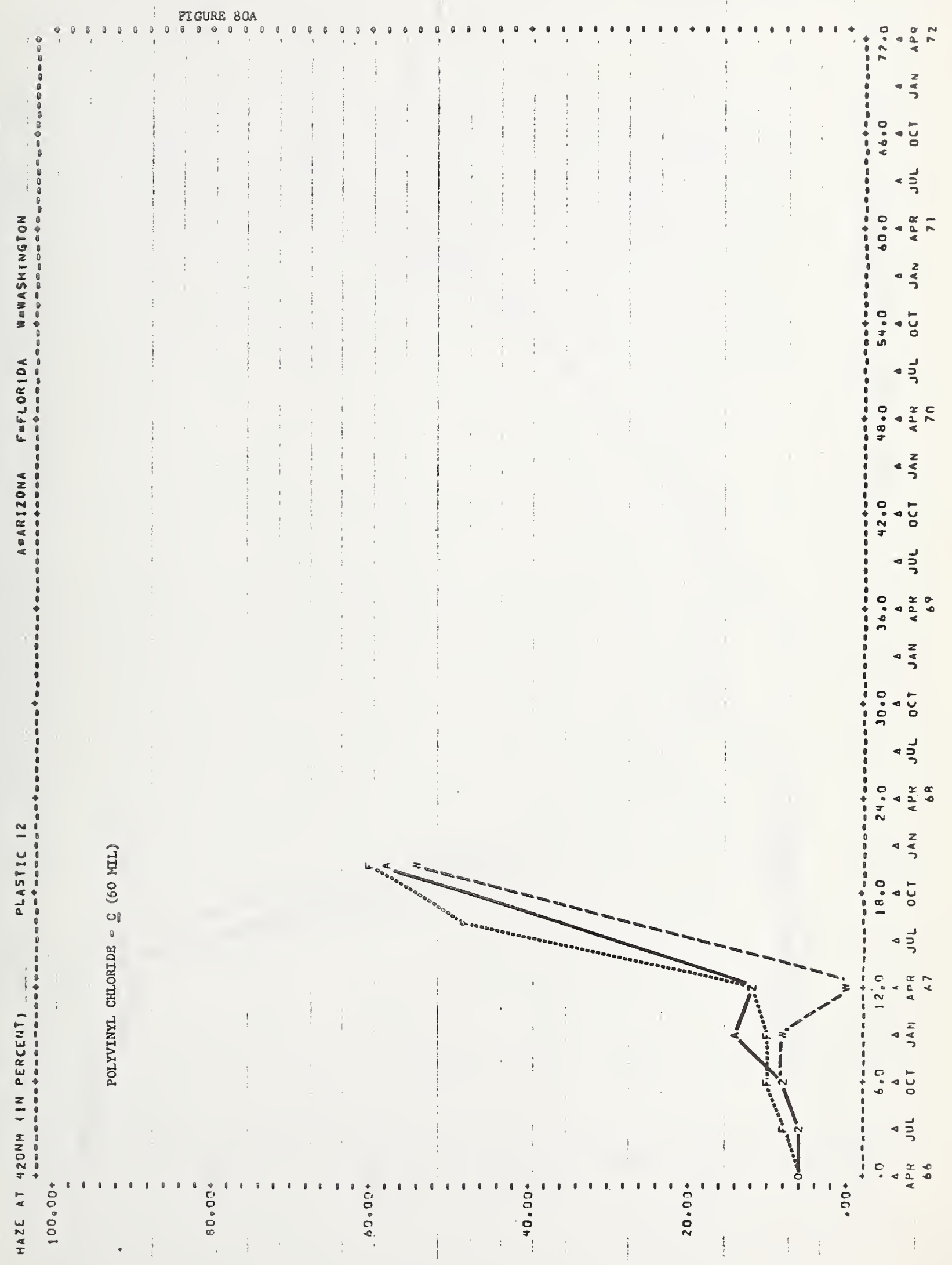


FIGURE $80 \mathrm{~B}$

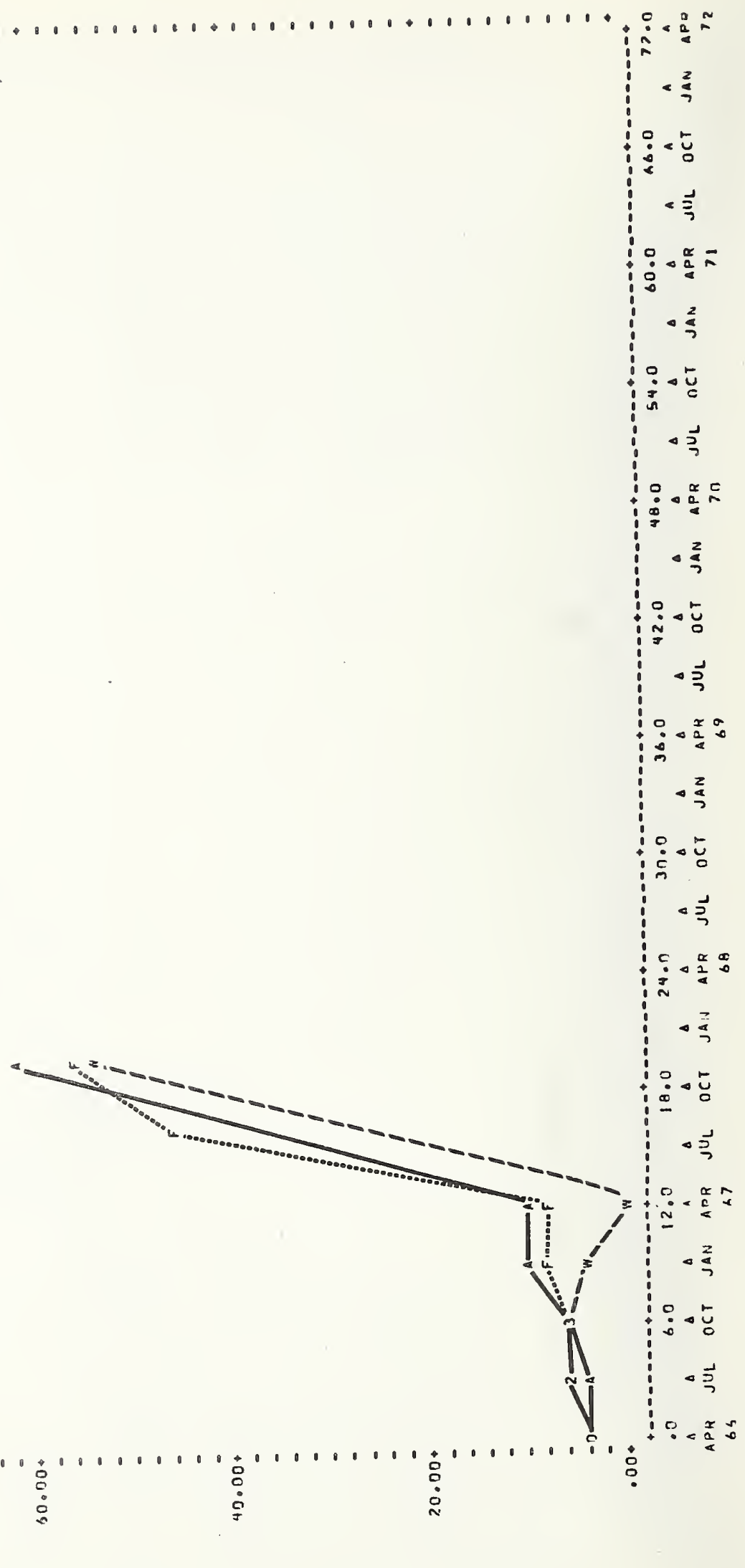


FIGURE BLA

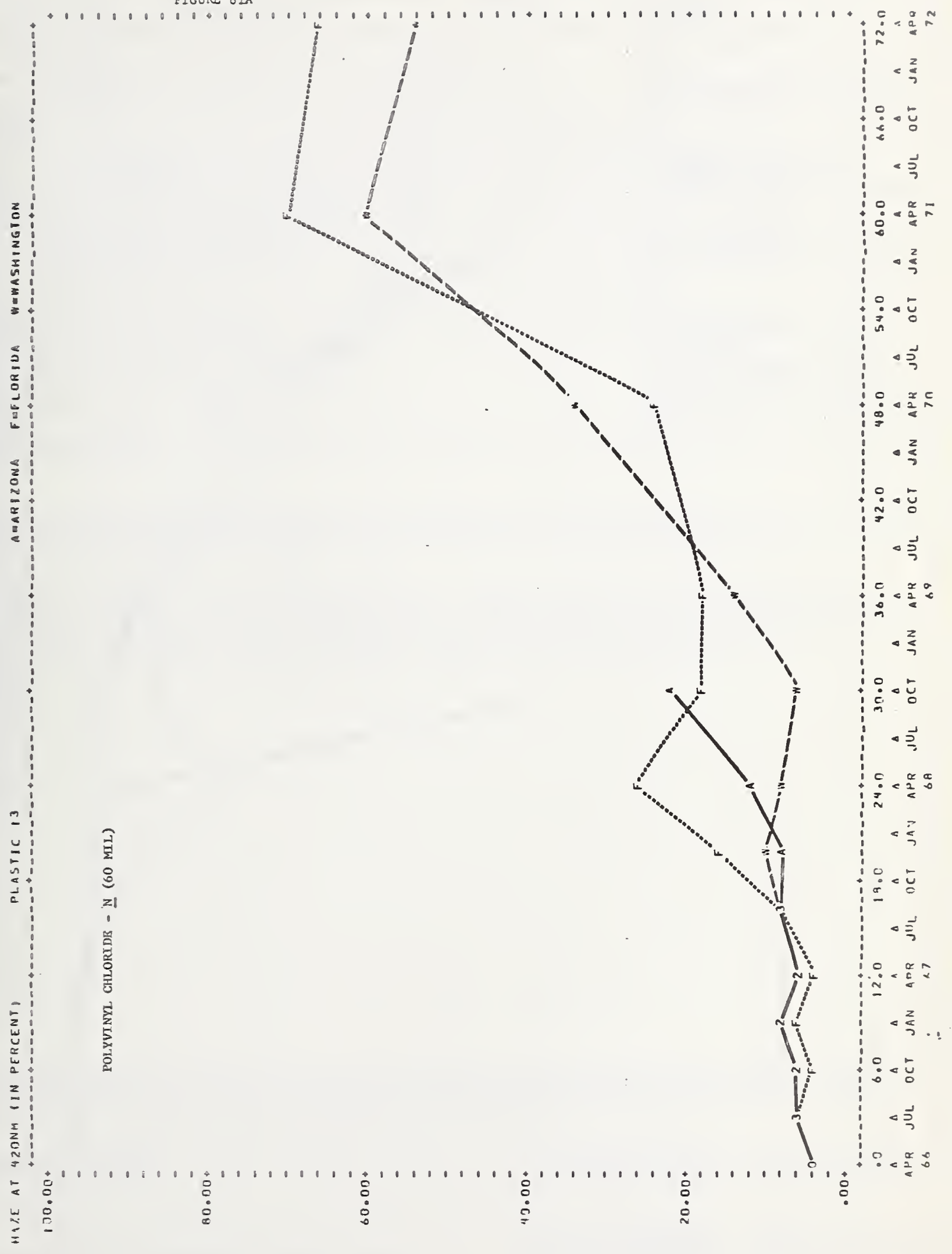


FIGURE $81 B$

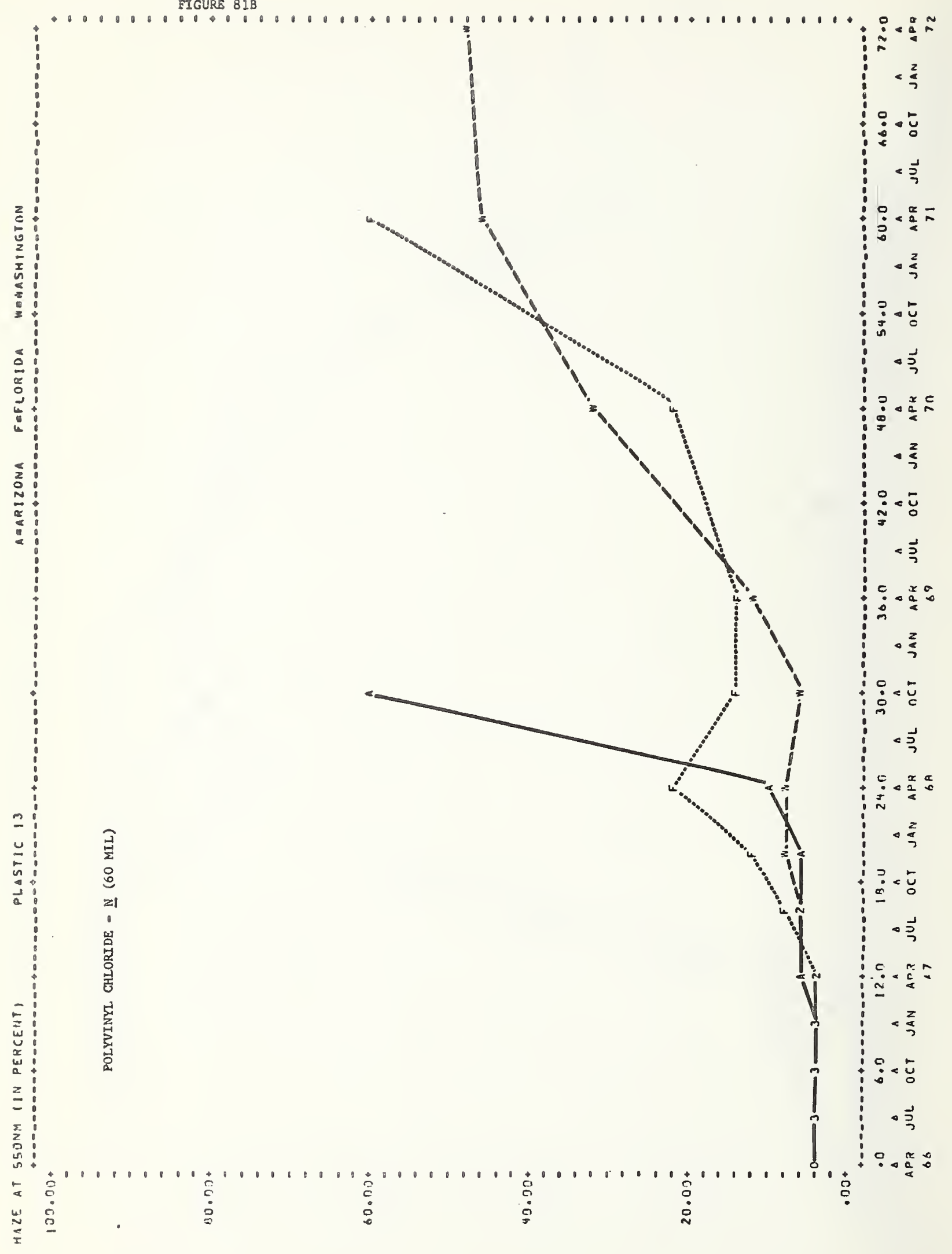


FOKIM NBS-114A $\{1-71\}$

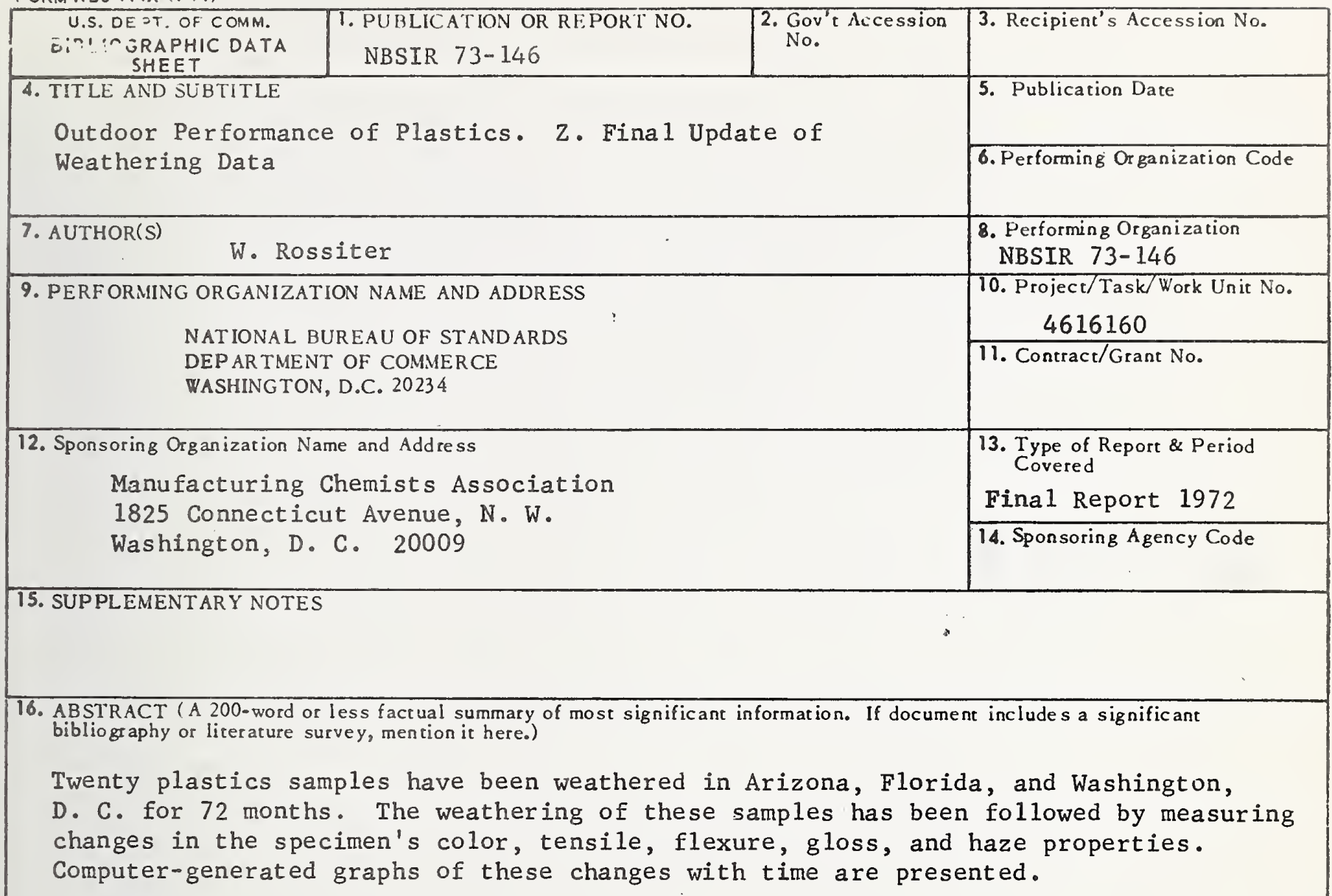

17. KEY WORDS (Alphabetical order, separated by semicolons) Manufacturing Chemists Association (MCS); performance of plastics outdoors; plastics; weathering of plastics

18. AVAILABILITY STATEMENT

[X] UNL IMTED.

$\square$ FOR OFFICIAL DISTRIBUTION. DO NOT RELEASE TO NTIS.

\begin{tabular}{|l|c|}
\hline $\begin{array}{l}\text { 19. SECURITY CLASS } \\
\text { (THIS REPORT) }\end{array}$ & 21. NO. OF PAGES \\
UNCL ASSIFIED & $\therefore$ \\
\hline $\begin{array}{l}\text { 20. SECURITY CLASS } \\
\text { (THIS PAGE) }\end{array}$ & 22. Price \\
UNCI.ASSIFIED & \\
\hline
\end{tabular}




